Supporting Information for:

\title{
Construction of Cyclic Sulfamidates Bearing Two gem-Diaryl Stereocenters through a Rhodium-Catalyzed Stepwise Asymmetric Arylation Protocol
}

\author{
Yu-Fang Zhang, Diao Chen, Wen-Wen Chen and Ming-Hua $\mathrm{Xu}^{*}$ \\ State Key Laboratory of Drug Research, Shanghai Institute of Materia \\ Medica, Chinese Academy of Sciences, 555 Zuchongzhi Road, \\ Shanghai 201203, China \\ E-mail:xumh@simm.ac.cn
}

Table of Contents

1. General.

2. General procedure for Rh-catalyzed 1,4-addition of $\alpha, \beta$-unsaturated cyclic ketimines 1 .

3. General procedure for Rh-catalyzed 1,2-addition of ketimines $3 a . \ldots \ldots \ldots \ldots \ldots . . . . .52$

4. Procedure for Rh-catalyzed arylation of ketimine $6 \ldots \ldots \ldots \ldots \ldots \ldots \ldots \ldots \ldots \ldots$

5. Characterization data and HPLC chromatogram of addition products............S4

6. Synthetic transformations of arylation products ..................................................S33

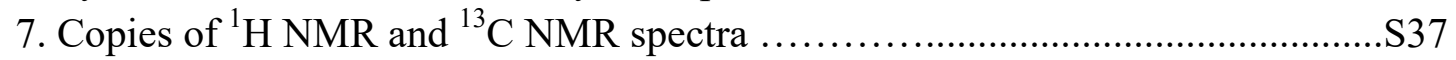




\section{General}

All anaerobic and moisture-sensitive manipulations were carried out with standard Schlenk techniques under pre-dried nitrogen or argon. Solvents were dried and distilled by standard procedures. NMR spectra were recorded on a Mercury 300 spectrometer $\left(300 \mathrm{MHz}\right.$ for $\left.{ }^{1} \mathrm{H}\right)$, and Varian spectrometer (400 $\mathrm{MHz}$ for ${ }^{1} \mathrm{H}, 100 \mathrm{MHz}$ or $125 \mathrm{MHz}$ for ${ }^{13} \mathrm{C}$ ). Chemical shifts are reported in $\delta$ ppm referenced to an internal $\mathrm{SiMe}_{4}$ standard for ${ }^{1} \mathrm{H}$ NMR and chloroform- $d(\delta 77.36)$ for ${ }^{13} \mathrm{C}$ NMR. HRMS were recorded on a Q-TOF mass spectrometer with ESI resource or Magnetic Sector for EI. Optical rotations were measured on a Perkin-Elmer 241 MC polarimeter. HPLC was performed on a JASCO 2000 instrument by using Daicel columns with 2-propanol/hexane as the eluent.

\section{General procedure for $\mathrm{Rh}$-catalyzed 1,4-addition of $\alpha, \beta$-unsaturated cyclic ketimines 1}

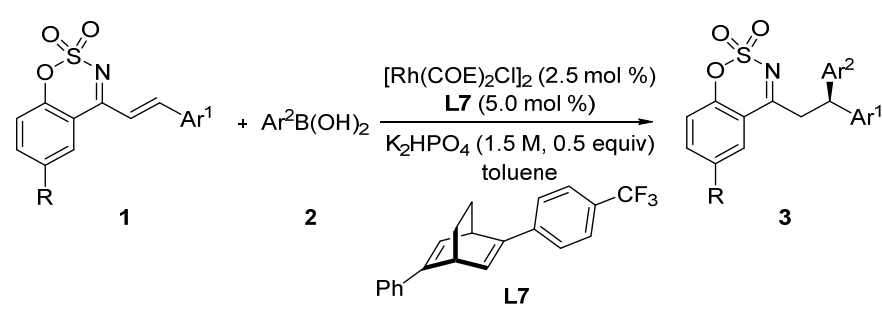

Under $\mathrm{Ar}$ atmosphere, a solution of substrate $\alpha, \beta$-unsaturated ketimine $1(0.20 \mathrm{mmol}),\left[\mathrm{Rh}(\mathrm{COE})_{2} \mathrm{Cl}\right]_{2}$ ( $2.5 \mathrm{~mol} \%, 3.6 \mathrm{mg}, 0.01 \mathrm{mmol}$ of Rh), ligand $\mathbf{L} 7(5.0 \mathrm{~mol} \%, 3.2 \mathrm{mg}, 0.01 \mathrm{mmol}$ ), and arylboronic acid $(0.40 \mathrm{mmol})$ in $1.0 \mathrm{~mL}$ of toluene was stirred at room temperature for $30 \mathrm{~min}$. To this mixture was added aqueous $\mathrm{K}_{2} \mathrm{HPO}_{4}(66 \mu \mathrm{L}, 1.5 \mathrm{M}, 0.10 \mathrm{mmol})$ and then the resulting mixture was stirred at room temperature (unless noted otherwise) for 12 hours. The solvent was removed under reduced pressure and the residue was purified by silica gel column chromatography using petroleum ether/ethyl acetate as an eluent to afford the corresponding addition product $\mathbf{3}$ (Note: For HPLC reference, $( \pm$ )- $\mathbf{3}$ was obtained with $\left[\mathrm{Rh}(\mathrm{COD})_{2} \mathrm{Cl}\right]_{2}$ as catalyst under the same conditions).

\section{General procedure for Rh-catalyzed 1,2-addition of ketimine 3}
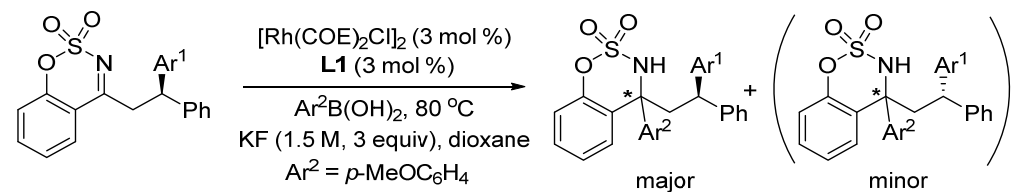

3<smiles>CC(C)(C)S(=O)NCC(=[IH])C[Pb]#N</smiles>

$4 a$

$4 a^{\prime}$

Under $\mathrm{Ar}$ atmosphere, a solution of substrate ketimine $3(0.10 \mathrm{mmol}),\left[\mathrm{Rh}(\mathrm{COE})_{2} \mathrm{Cl}\right]_{2}(1.5 \mathrm{~mol} \%, 1.1$ $\mathrm{mg}, 0.003 \mathrm{mmol}$ of Rh), ligand $\mathbf{L} \mathbf{1}(1.0 \mathrm{mg}, 0.003 \mathrm{mmol})$, and arylboronic acid $\mathbf{2 b}(0.20 \mathrm{mmol})$ in 1.0 $\mathrm{mL}$ of toluene was stirred at room temperature for $30 \mathrm{~min}$. To this mixture was added aqueous $\mathrm{KF}(0.2$ $\mathrm{mL}, 1.5 \mathrm{M}, 0.30 \mathrm{mmol}$ ) and then the resulting mixture was stirred at $80^{\circ} \mathrm{C}$ (unless noted otherwise) for 12 hours. The solvent was removed under reduced pressure and the residue was purified by silica gel column chromatography using petroleum ether/ethyl acetate as an eluent to afford the corresponding addition product 4 . 


\section{Procedure for Rh-catalyzed arylation of ketimine 6}

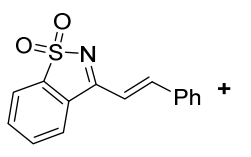

6

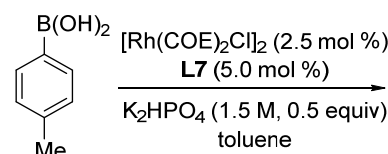

2a

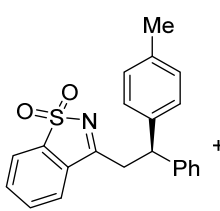

7

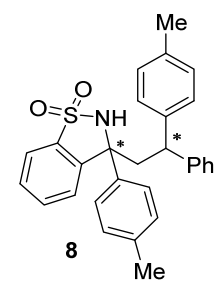

Under $\mathrm{Ar}$ atmosphere, a solution of substrate $\alpha, \beta$-unsaturated ketimine $6(0.20 \mathrm{mmol}),\left[\mathrm{Rh}(\mathrm{COE})_{2} \mathrm{Cl}\right]_{2}$ ( $2.5 \mathrm{~mol} \%, 3.6 \mathrm{mg}, 0.01 \mathrm{mmol}$ of Rh), ligand $\mathbf{L} 7$ (5.0 mol \%, $3.2 \mathrm{mg}, 0.01 \mathrm{mmol}$ ), and p-tolylboronic acid $2 \mathrm{a}(0.40 \mathrm{mmol}, 2$ equiv) in $1.0 \mathrm{~mL}$ of toluene was stirred at room temperature for $30 \mathrm{~min}$. To this mixture was added aqueous $\mathrm{K}_{2} \mathrm{HPO}_{4}(66 \mu \mathrm{L}, 1.5 \mathrm{M}, 0.10 \mathrm{mmol})$ and then the resulting mixture was stirred at room temperature for 12 hours. The solvent was removed under reduced pressure and the residue was purified by silica gel column chromatography using petroleum ether/ethyl acetate as an eluent to afford 1,4-adduct $\mathbf{7}$ and double arylation product $\mathbf{8}$ respectively (Note: For HPLC reference, $( \pm)-7$ was obtained with $\left[\mathrm{Rh}(\mathrm{COD})_{2} \mathrm{Cl}\right]_{2}$ as catalyst under the same conditions).

Double arylation product 8 was obtained as the sole product in the presence of 3 equiv of p-tolylboronic acid $2 \mathrm{a}(0.60 \mathrm{~mol})$ for $24 \mathrm{~h}$. 


\section{Characterization data and HPLC of addition products}

(R)-4-(2-phenyl-2-(p-tolyl)ethyl)benzo[e][1,2,3]oxathiazine 2,2-dioxide (3a)

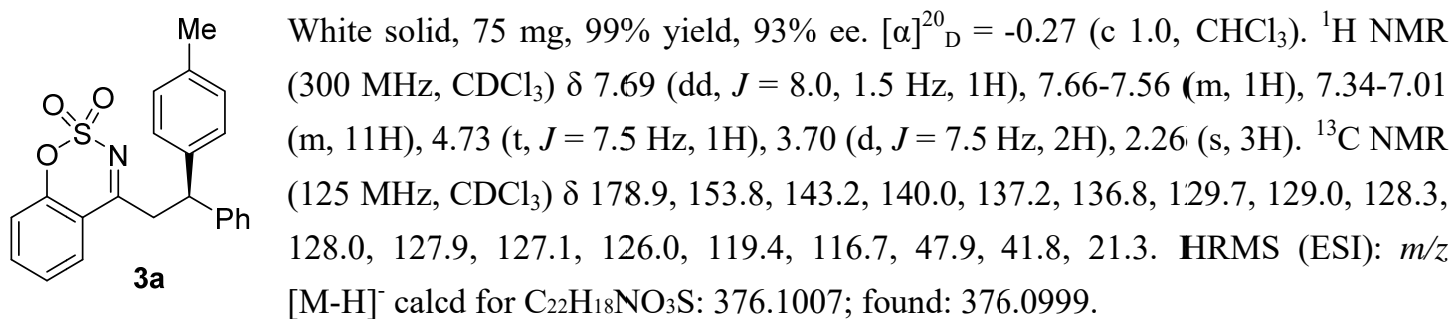

HPLC: Chiralpak IC column $(250 \mathrm{~mm})$; detected at $224 \mathrm{~nm}$; hexane/i-propanol = 80/20; flow $=0.7$ $\mathrm{mL} / \mathrm{min}$; Retention time: $25.3 \mathrm{~min}$ (major), $28.8 \mathrm{~min}$. 93\% ee.

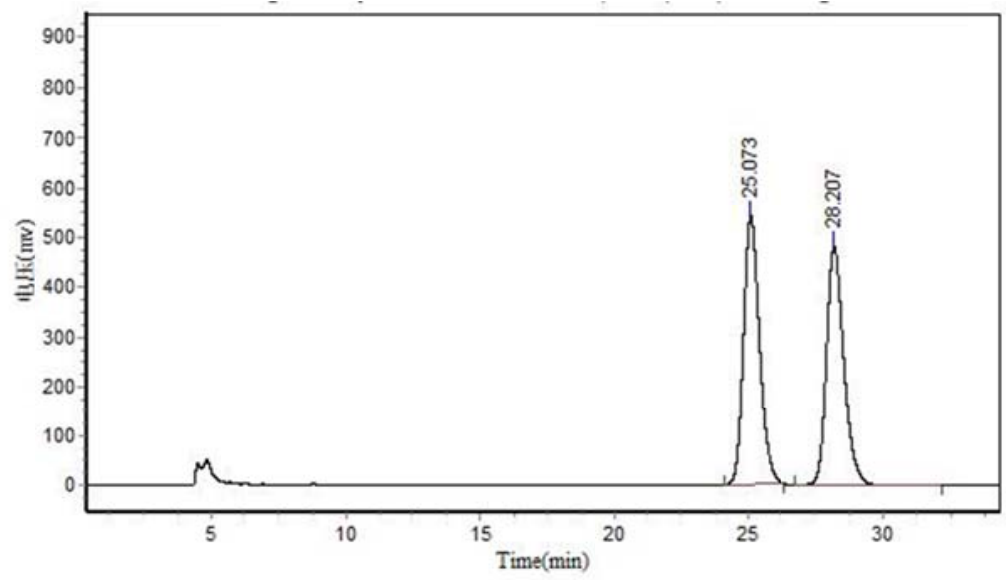

Results

\begin{tabular}{|c|c|c|c|c|c|}
\hline \multicolumn{6}{|c|}{ (1) } \\
\hline Peak No. & Peak ID & Ret Time & Height & Area & Conc. \\
\hline 1 & & 25.073 & 541697.438 & 22517524.000 & 50.7869 \\
\hline 2 & & 28.207 & 482062.719 & 21819736.000 & 49.2131 \\
\hline Total & & & 1023760.156 & 44337260.000 & 100.0000 \\
\hline
\end{tabular}

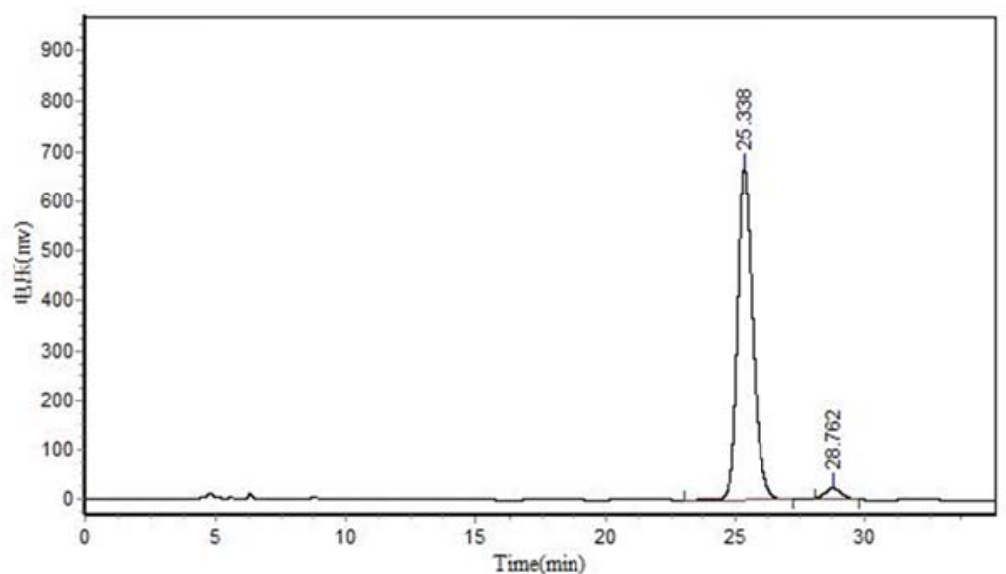

Results

\begin{tabular}{|c|c|c|c|c|c|}
\hline Peak No. & Peak ID & Ret Time & Height & Area & Conc. \\
\hline 1 & & 25.338 & 668640.438 & 27930202.000 & 96.5807 \\
\hline 2 & & 28.762 & 22467.125 & 988814.125 & 3.4193 \\
\hline Total & & & 691107.563 & 28919016.125 & 100.0000 \\
\hline
\end{tabular}


(R)-4-(2-(4-methoxyphenyl)-2-phenylethyl)benzo[e][1,2,3]oxathiazine 2,2-dioxide (3b)

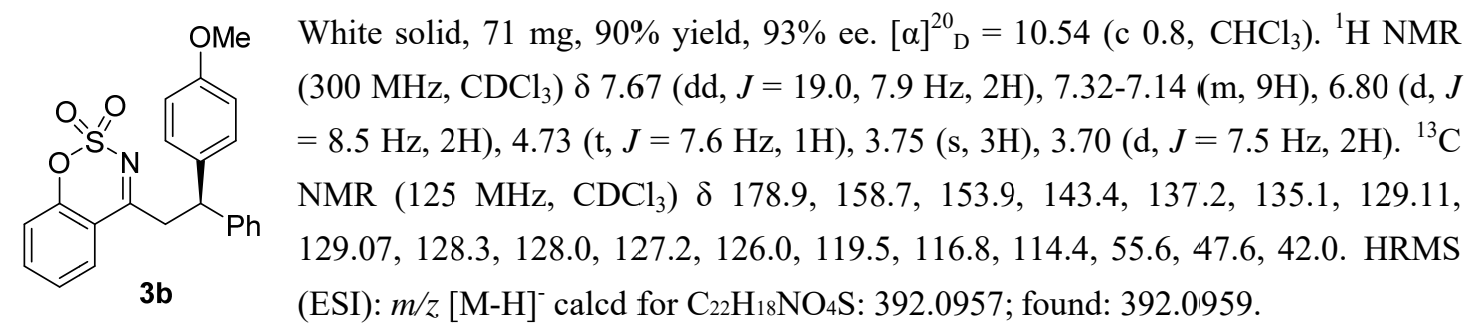

HPLC: Chiralpak AD-H column $(250 \mathrm{~mm})$; detected at $224 \mathrm{~nm}$; hexane/i-propanol = 95/5; flow = 1.0 $\mathrm{mL} / \mathrm{min}$; Retention time: $36.4 \mathrm{~min}$ (major), $41.6 \mathrm{~min} .93 \%$ ee.

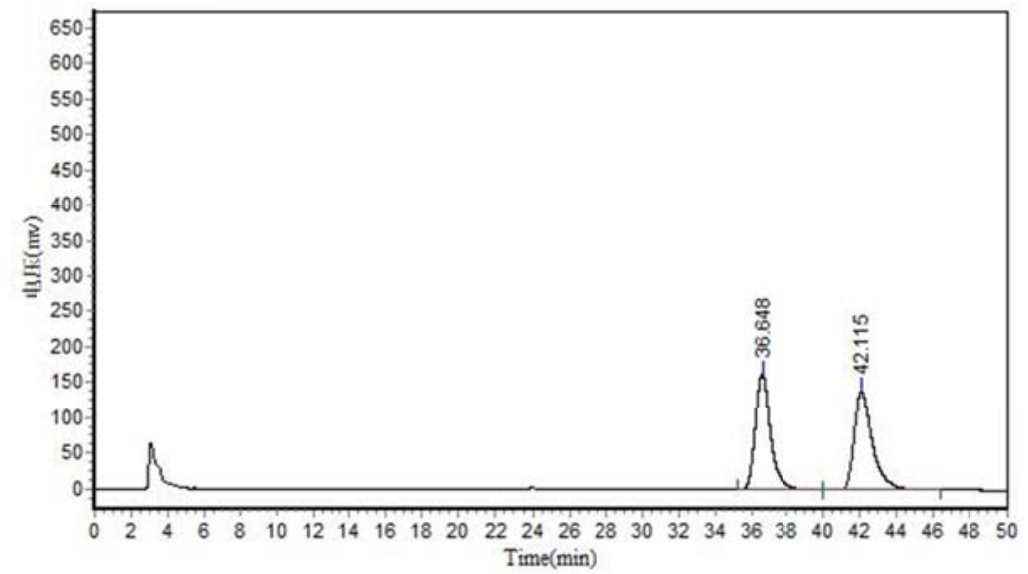

Results

\begin{tabular}{cccccc} 
Peak No. & Peak ID & Ret Time & Height & Area & Conc. \\
\hline 1 & 36.648 & 163031.859 & 9663607.000 & 49.8227 \\
2 & 42.115 & 137787.719 & 9732368.000 & 50.1773 \\
\hline Total & & 300819.578 & 19395975.000 & 100.0000
\end{tabular}

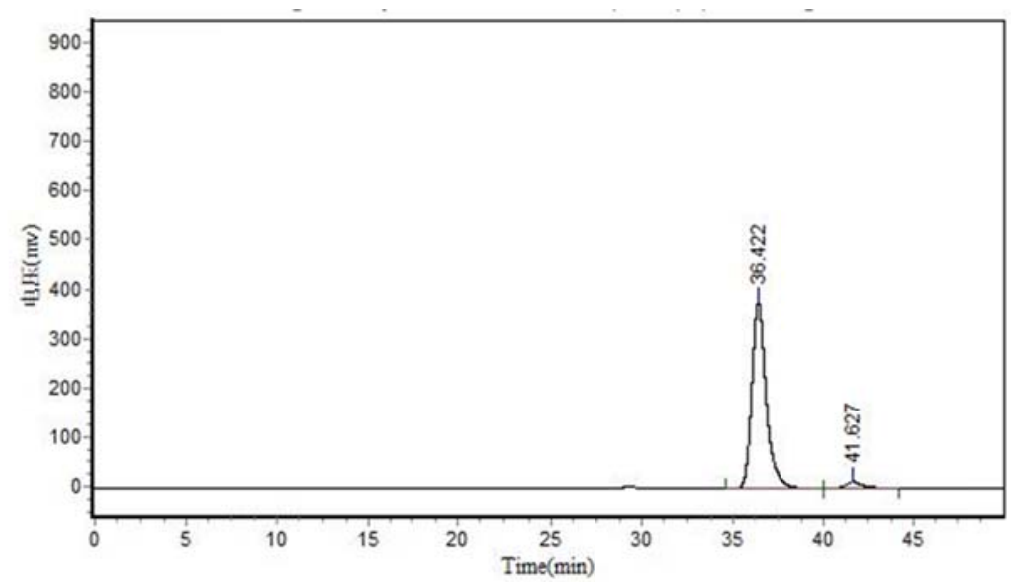

\begin{tabular}{|c|c|c|c|c|c|}
\hline \multicolumn{6}{|c|}{ Results } \\
\hline Peak No. & Peak ID & Ret Time & Height & Area & Conc. \\
\hline 1 & & 36.422 & 375897.531 & 20515006.000 & 96.3174 \\
\hline 2 & & 41.627 & 12591.521 & 784375.188 & 3.6826 \\
\hline Total & & & 388489.052 & 21299381.188 & 100.0000 \\
\hline
\end{tabular}


(R)-4-(2-(4-chlorophenyl)-2-phenylethyl)benzo[e][1,2,3]oxathiazine 2,2-dioxide (3c)

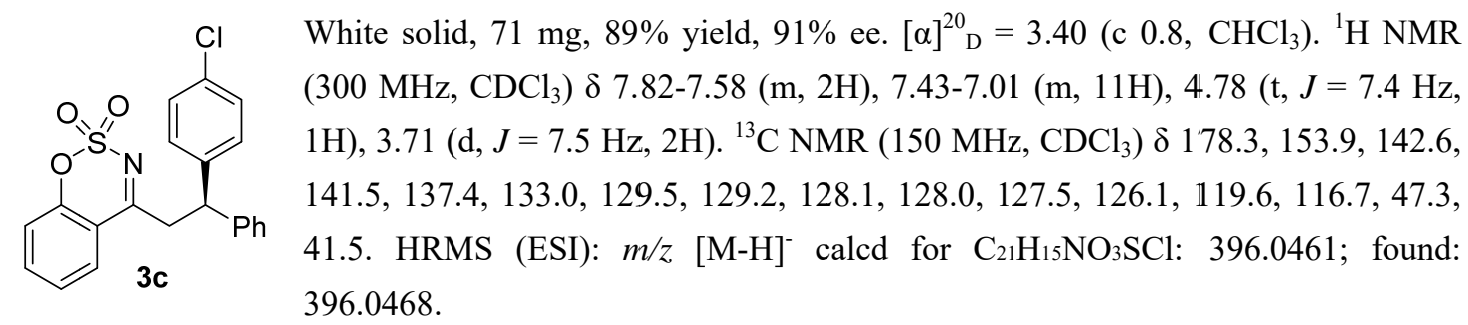

HPLC: Chiralpak IC column $(250 \mathrm{~mm})$; detected at $224 \mathrm{~nm}$; hexane $/$ - -propanol $=85 / 15$; flow $=0.8$ $\mathrm{mL} / \mathrm{min}$; Retention time: $34.3 \mathrm{~min}$ (major), $39.0 \mathrm{~min} .91 \%$ ee.

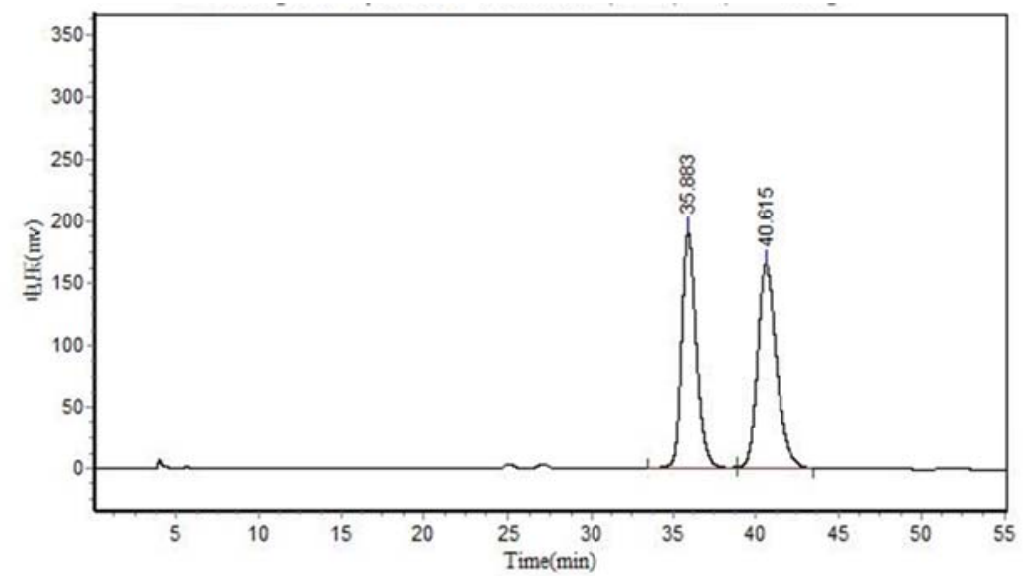

\begin{tabular}{cccccc} 
& \multicolumn{2}{c}{ Results } & Area & Conc. \\
\hline Peak No. & Peak ID & Ret Time & Height & 11847477.000 & 47.0783 \\
2 & 35.883 & 191531.781 & 13317980.000 & 52.9217 \\
\hline Total & 40.615 & 165094.000 & 25165457.000 & 100.0000
\end{tabular}

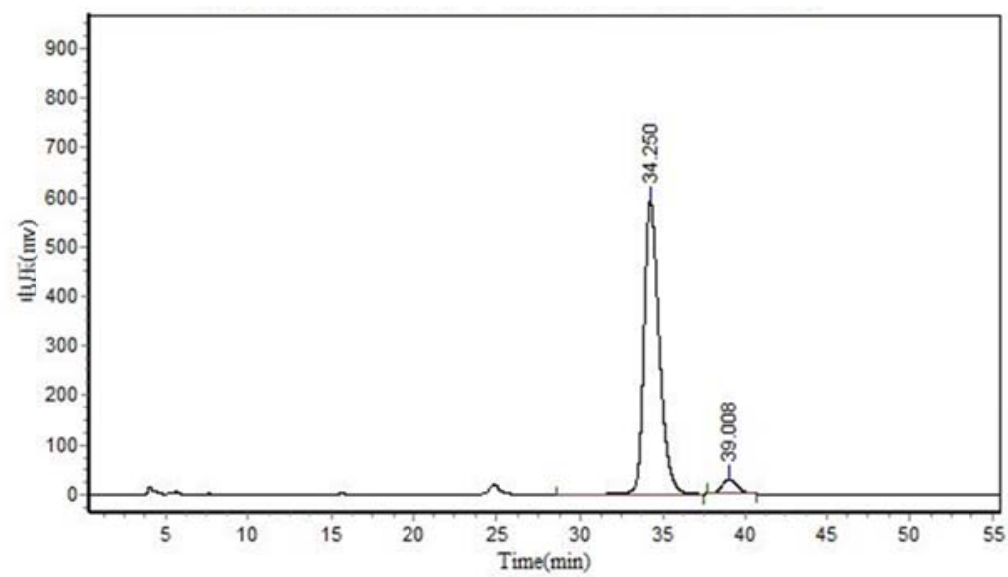

\begin{tabular}{|c|c|c|c|c|c|}
\hline \multicolumn{6}{|c|}{ Results } \\
\hline Peak No. & Peak ID & Ret Time & Height & Area & Conc. \\
\hline 1 & & 34.250 & 592365.875 & 37848380.000 & 95.2943 \\
\hline 2 & & 39.008 & 28159.246 & 1868996.875 & 4.7057 \\
\hline Total & & & 620525.121 & 39717376.875 & 100.0000 \\
\hline
\end{tabular}


(R)-4-(2-phenyl-2-(o-tolyl)ethyl)benzo[e][1,2,3]oxathiazine 2,2-dioxide (3d)<smiles>Cc1ccccc1C(CC1=NS(=O)(=O)Oc2ccccc21)c1ccccc1</smiles>

White solid, $72 \mathrm{mg}, 96 \%$ yield, $99 \%$ ee. $[\alpha]^{20}{ }_{\mathrm{D}}=-127.98\left(\mathrm{c} 1.0, \mathrm{CHCl}_{3}\right) .{ }^{1} \mathrm{H}$ NMR $\left(300 \mathrm{MHz}, \mathrm{CDCl}_{3}\right) \delta$ 7.68-7.62 (m, 2H), 7.33-7.13 (m, 11H), $4.96(\mathrm{t}, J=$ $7.4 \mathrm{~Hz}, 1 \mathrm{H}), 3.73$ (d, $J=7.5 \mathrm{~Hz}, 2 \mathrm{H}), 2.26$ (s, 3H). ${ }^{13} \mathrm{C} \mathrm{NMR}\left(125 \mathrm{MHz}, \mathrm{CDCl}_{3}\right)$ $\delta 179.0,153.9,142.6,140.8,137.2,136.9,131.4,129.0,128.3,128.2,127.2$, 127.1, 126.5, 126.4, 126.0, 119.5, 116.9, 44.3, 42.1, 20.2. HRMS (ESI): $\mathrm{m} / \mathrm{z}$ [M-H] $]^{-}$calcd for $\mathrm{C}_{22} \mathrm{H}_{18} \mathrm{NO}_{3} \mathrm{~S}$ : 376.1007 ; found: 376.1007 .

HPLC: Chiralpak AY-H column (250 mm); detected at $224 \mathrm{~nm}$; hexane/i-propanol = 85/15; flow = 0.7 $\mathrm{mL} / \mathrm{min}$; Retention time: $27.4 \mathrm{~min}, 32.1 \mathrm{~min}$ (major). 99\% ee.

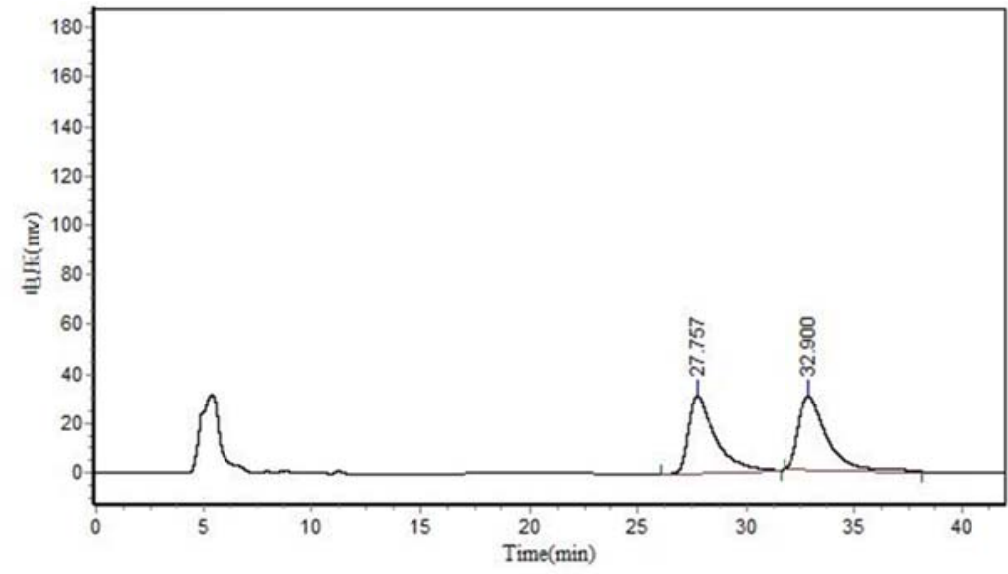

Results

\begin{tabular}{|c|c|c|c|c|c|}
\hline Peak No. & Peak ID & Ret Time & Height & Area & Conc. \\
\hline 1 & & 27.757 & 31453.678 & 2734849.500 & 49.8310 \\
\hline 2 & & 32.900 & 30129.199 & 2753394.750 & 50.1690 \\
\hline Total & & & 61582.877 & 5488244.250 & 100.0000 \\
\hline
\end{tabular}

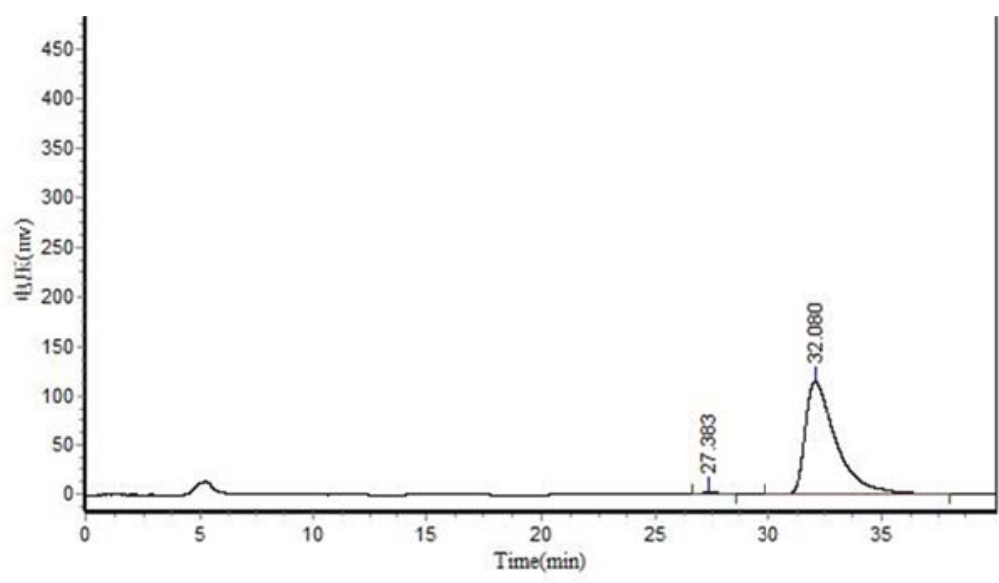

Results

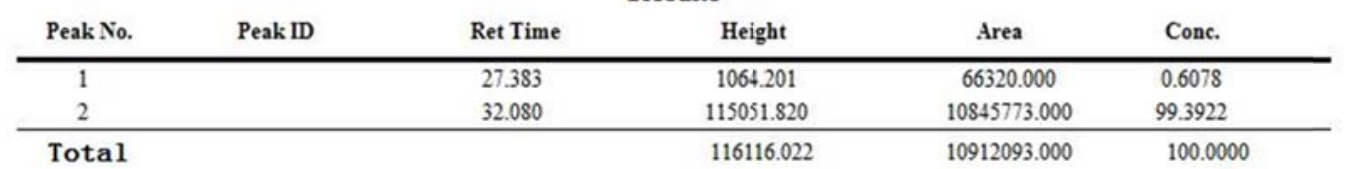


(R)-4-(2-(2-bromophenyl)-2-phenylethyl)benzo[e][1,2,3]oxathiazine 2,2-dioxide (3e)<smiles>O=S1(=O)N=C(CC(c2ccccc2)c2ccccc2Br)c2ccccc2O1</smiles>

White solid, $84 \mathrm{mg}, 95 \%$ yield, $99 \%$ ee. $[\alpha]^{20}{ }_{\mathrm{D}}=2.00\left(\mathrm{c} 1.0, \mathrm{CHCl}_{3}\right) .{ }^{1} \mathrm{H}$ NMR $\left(300 \mathrm{MHz}, \mathrm{CDCl}_{3}\right) \delta 7.78(\mathrm{~d}, J=7.9 \mathrm{~Hz}, 1 \mathrm{H}), 7.70-7.64(\mathrm{~m}, 1 \mathrm{H}), 7.54$ (d, $J=7.8$ $\mathrm{Hz}, 1 \mathrm{H}), 7.42-7.16(\mathrm{~m}, 9 \mathrm{H}), 7.09(\mathrm{~d}, J=3.7 \mathrm{~Hz}, 1 \mathrm{H}), 5.25(\mathbf{t}, J=7.4 \mathrm{~Hz}, 1 \mathrm{H})$, 3.88-3.58 (m, 2H). ${ }^{13} \mathrm{C}$ NMR (125 MHz, $\left.\mathrm{CDCl}_{3}\right) \delta 177.0,153.0,141.0,140.4$, $136.2,132.8,128.1,127.8,127.4,127.2,127.1,126.4,125.1,124.2,118.6,115.6$, 45.9, 40.5. HRMS (ESI): $\mathrm{m} / z$ [M-H] calcd for $\mathrm{C}_{21} \mathrm{H}_{15} \mathrm{NO}_{3} \mathrm{SBr}$ : 439.9956; found: 439.9963.

HPLC: Chiralpak AD-3 column $(250 \mathrm{~mm})$; detected at $224 \mathrm{~nm}$; hexane $/ \mathrm{i}$-propanol $=95 / 5$; flow $=0.7$ $\mathrm{mL} / \mathrm{min}$; Retention time: $39.6 \mathrm{~min}$ (major), $44.5 \mathrm{~min} .99 \%$ ee.

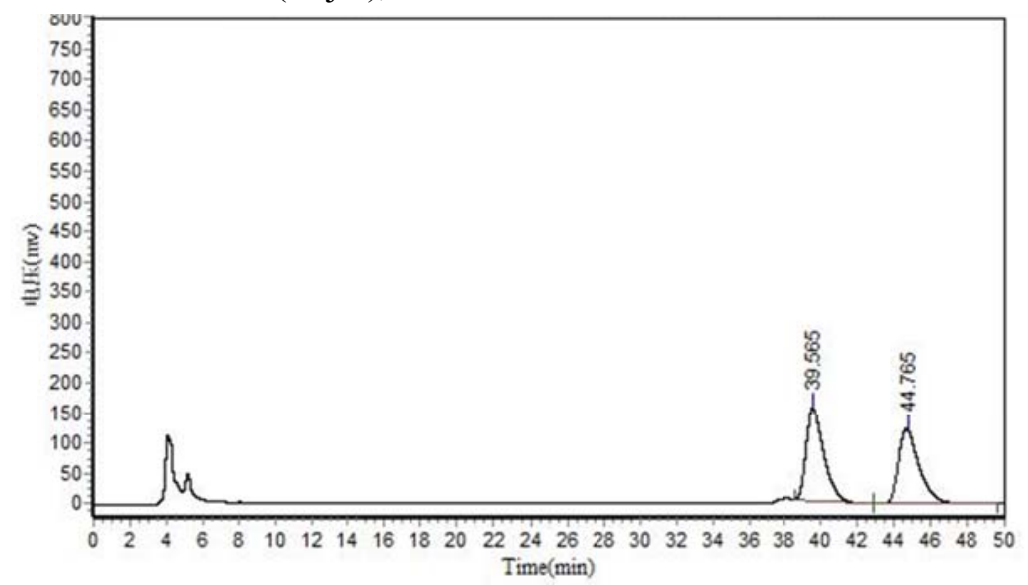

\section{Results}

\begin{tabular}{cccccc} 
Peak No. & Peak ID & Ret Time & Height & Area & Conc. \\
\hline 1 & 39.565 & 157563.969 & 11212930.000 & 53.0402 \\
2 & 44.765 & 123776.367 & 9927522.000 & 46.9598 \\
\hline Total & & 281340.336 & 21140452.000 & 100.0000
\end{tabular}

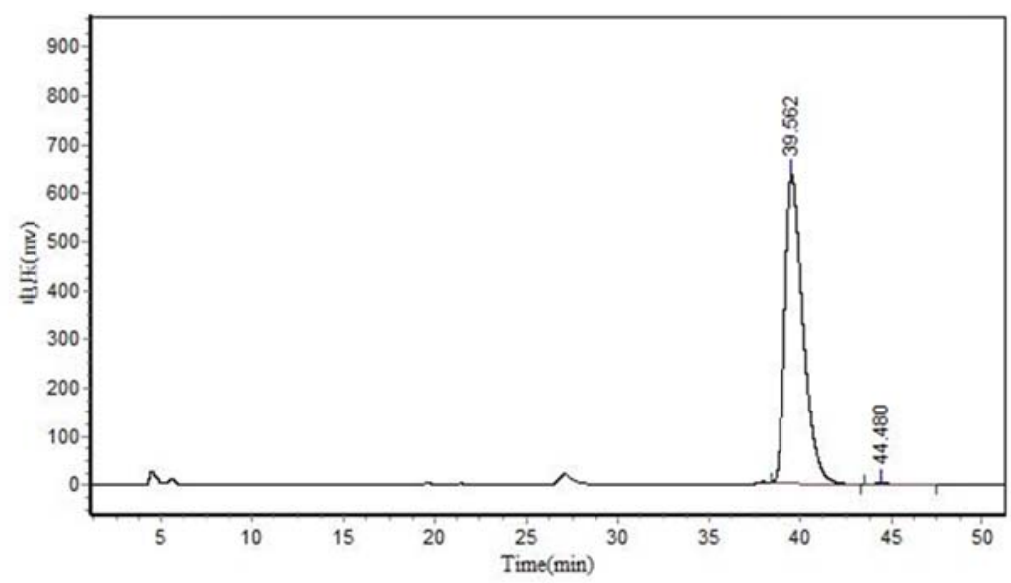

Results

\begin{tabular}{|c|c|c|c|c|c|}
\hline \multicolumn{6}{|c|}{200} \\
\hline Peak No. & Peak ID & Ret Time & Height & Area & Conc. \\
\hline 1 & & 39.562 & 632561.375 & 44032080.000 & 99.6892 \\
\hline 2 & & 44.480 & 1760.845 & 137286.531 & 0.3108 \\
\hline Total & & & 634322.220 & 44169366.531 & 100.0000 \\
\hline
\end{tabular}


(R)-4-(2-(2-fluorophenyl)-2-phenylethyl)benzo[e][1,2,3] oxathiazine 2,2-dioxide (3f)<smiles>O=S1(=O)N=C(CC(c2ccccc2)c2ccccc2F)c2ccccc2O1</smiles>

$3 f$

White solid, $68 \mathrm{mg}, 89 \%$ yield, $85 \%$ ee. $[\alpha]^{20}{ }_{\mathrm{D}}=-8.53$ (c $\left.1.0, \mathrm{CHCl}_{3}\right) .{ }^{1} \mathrm{H}$ NMR (300 MHz, $\left.\mathrm{CDCl}_{3}\right) \delta 7.80(\mathrm{~d}, J=7.9 \mathrm{~Hz}, 1 \mathrm{H}), 7.70-7.65(\mathrm{~m}, 1 \mathrm{H})$, 7.39-7.16 (m, 9H), 7.13-6.93 (m, 2H), $5.00(\mathrm{t}, J=7.5 \mathrm{~Hz}, 1 \mathrm{H}), 3.79$ (d, $J=7.6$ $\mathrm{Hz}, 2 \mathrm{H}) .{ }^{13} \mathrm{C} \mathrm{NMR}\left(125 \mathrm{MHz}, \mathrm{CDCl}_{3}\right) \delta 178.2,160.9\left(\mathrm{~d}, J_{\mathrm{CF}}=245 \mathrm{~Hz}\right), 153.9$, $141.6,137.2,129.8\left(\mathrm{~d}, J_{\mathrm{CF}}=13.8 \mathrm{~Hz}\right), 129.6\left(\mathrm{~d}, J_{\mathrm{CF}}=3.8 \mathrm{~Hz}\right), 129.12,129.06$, 128.1, 127.5, 126.1, $124.8\left(\mathrm{~d}, J_{\mathrm{CF}}=3.8 \mathrm{~Hz}\right), 119.6,116.3\left(\mathrm{~d}, J_{\mathrm{CF}}=21.3 \mathrm{~Hz}\right), 42.4,40.6$. HRMS (ESI): $\mathrm{m} / \mathrm{z}[\mathrm{M}-\mathrm{H}]^{-}$calcd for $\mathrm{C}_{21} \mathrm{H}_{15} \mathrm{NO}_{3} \mathrm{SF}: 380.0757$; found: 380.0754 .

HPLC: Chiralpak IC column $(250 \mathrm{~mm})$; detected at $224 \mathrm{~nm}$; hexane $/$ - -propanol $=85 / 15$; flow $=0.8$ $\mathrm{mL} / \mathrm{min}$; Retention time: $38.5 \mathrm{~min}$ (major), $45.1 \mathrm{~min} .85 \%$ ee.

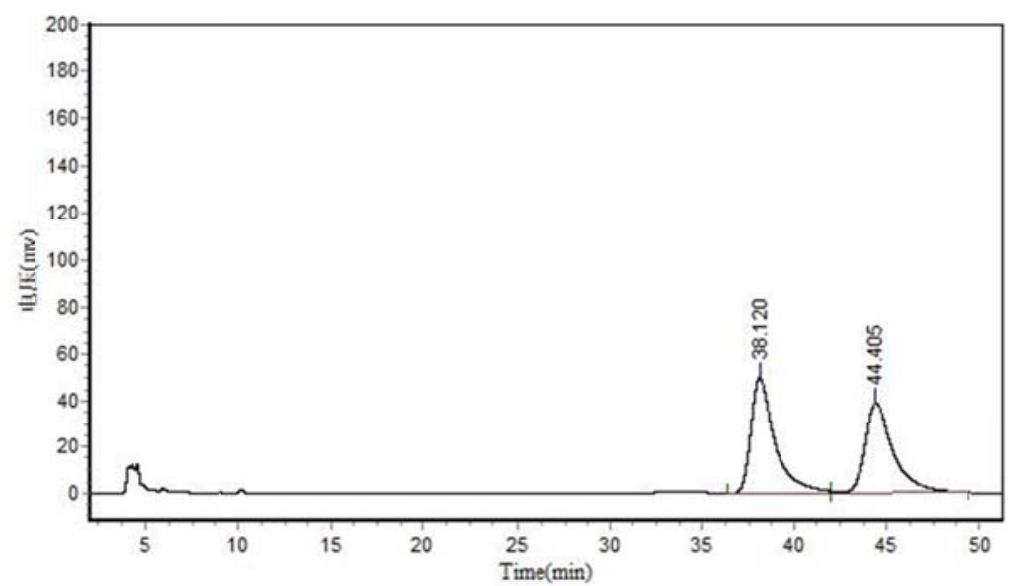

\begin{tabular}{cccccc} 
& \multicolumn{2}{c}{ Results } & Area & Conc. \\
\hline Peak No. & Peak ID & Ret Time & Height & 4530980.000 & 52.5626 \\
2 & 38.120 & 49739.223 & 4089175.000 & 47.4374 \\
\hline Total & 44.405 & 38704.566 & $\$ 620155.000$ & 100.0000
\end{tabular}

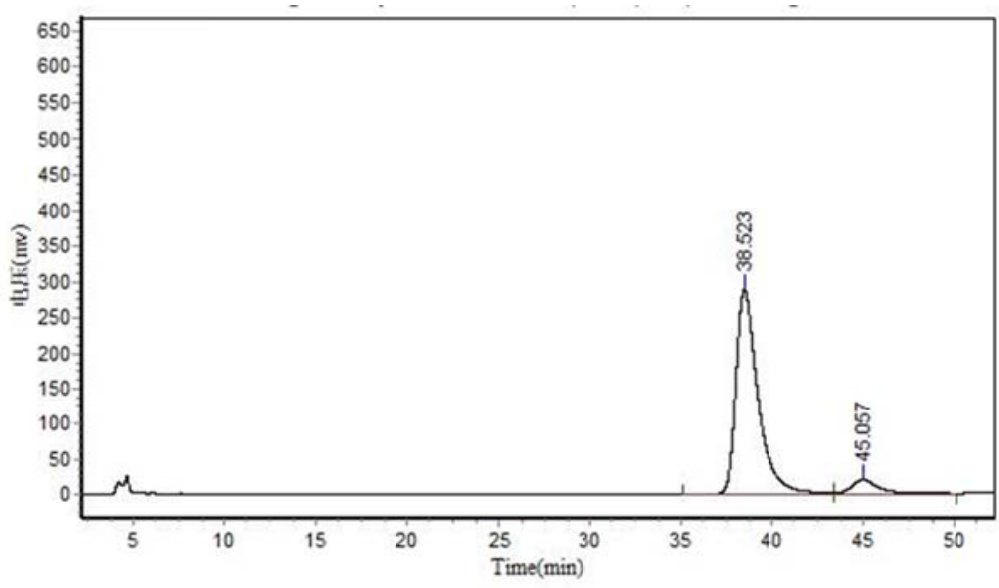

Results

\begin{tabular}{|c|c|c|c|c|c|}
\hline \multicolumn{6}{|c|}{ Results } \\
\hline Peak No. & Peak ID & Ret Time & Height & Area & Conc. \\
\hline 1 & & 38.523 & 287449.938 & 24472030.000 & 92.6213 \\
\hline 2 & & 45.057 & 19599.770 & 1949557.750 & 7.3787 \\
\hline Total & & & 307049.707 & 26421587.750 & 100.0000 \\
\hline
\end{tabular}


(R)-4-(2-(naphthalen-1-yl)-2-phenylethyl)benzo[e][1,2,3]oxathiazine 2,2-dioxide (3g)<smiles>O=S1(=O)N=C(CC(c2ccccc2)c2cccc3ccccc23)c2ccccc2O1</smiles>

White solid, $81 \mathrm{mg}, 98 \%$ yield, $99 \%$ ee. $[\alpha]^{20}=-87.53\left(\right.$ c $\left.1.0, \mathrm{CHCl}_{3}\right) .{ }^{1} \mathrm{H}$ NMR $\left(300 \mathrm{MHz}, \mathrm{CDCl}_{3}\right) \delta$ 8.16-7.99 (m, 1H), 7.91-7.79 (m, 1H), 7.79-7.70 (m, 1H), 7.64-7.59 (m, 2H), 7.53-7.39 (m, 4H), 7.34-7.18 (m, 6H), $7.14(\mathrm{~d}, J=6.8$ $\mathrm{Hz}, 1 \mathrm{H}), 5.69-5.46$ (t, $J=7.1 \mathrm{~Hz}, 1 \mathrm{H}), 3.94-3.76(\mathrm{~m}, 2 \mathrm{H}) .{ }^{13} \mathrm{C}$ NMR $(125 \mathrm{MHz}$, $\left.\mathrm{CDCl}_{3}\right) \delta 179.0,153.8,142.8,138.7,137.2,134.5,131.7,129.2,129.1,128.23$, $128.20,128.1,127.3,126.8,126.1,126.0,125.5,124.7,123.9,119.5,116.9,44.1,42.0$. HRMS (ESI): $\mathrm{m} / \mathrm{z}$ [M-H] $]^{-}$calcd for $\mathrm{C}_{25} \mathrm{H}_{18} \mathrm{NO}_{3} \mathrm{~S}: 412.1007$; found: 412.1010 .

HPLC: Chiralpak AD-H column $(250 \mathrm{~mm})$; detected at $224 \mathrm{~nm}$; hexane $/ i$-propanol = 95/5; flow $=0.7$ $\mathrm{mL} / \mathrm{min}$; Retention time: $23.6 \mathrm{~min}$ (major), $30.4 \mathrm{~min} .99 \%$ ee.

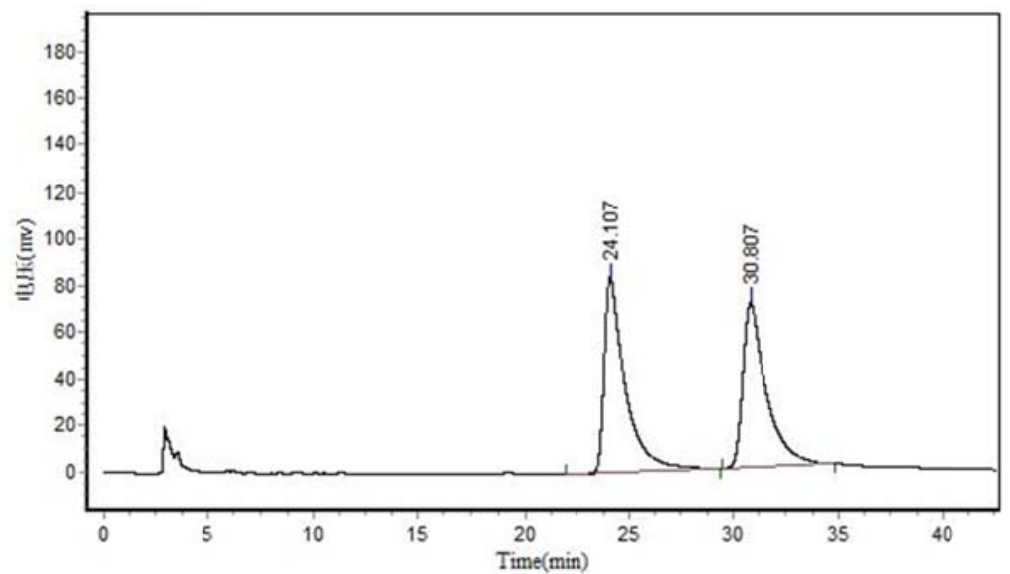

\begin{tabular}{|c|c|c|c|c|c|}
\hline \multicolumn{6}{|c|}{ Results } \\
\hline Peak No. & Peak ID & Ret Time & Height & Area & Conc. \\
\hline 1 & & 24.107 & $\$ 4462.500$ & 6316089.000 & 49.7665 \\
\hline 2 & & 30.807 & 72804.930 & 6375358.500 & 50.2335 \\
\hline Total & & & 157267.430 & 12691447.500 & 100.0000 \\
\hline
\end{tabular}

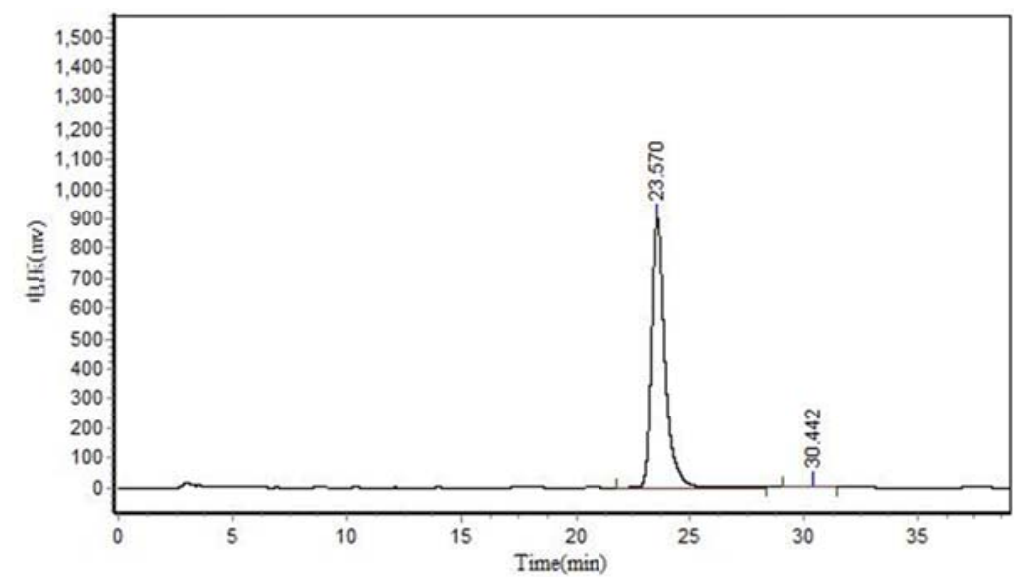

Results

\begin{tabular}{|c|c|c|c|c|c|}
\hline Peak No. & Peak ID & Ret Time & Height & Area & Conc. \\
\hline 1 & & 23.570 & 900629.875 & 36663632.000 & 99.6883 \\
\hline 2 & & 30.442 & 2123.066 & 114637.406 & 0.3117 \\
\hline Total & & & 902752.941 & 36778269.406 & 100.0000 \\
\hline
\end{tabular}


(R)-4-(2-(naphthalen-2-yl)-2-phenylethyl)benzo[e][1,2,3]oxathiazine 2,2-dioxide (3h)<smiles>O=S1(=O)Oc2ccccc2C(CC(c2ccccc2)c2ccc3ccccc3c2)=[SH]1</smiles>

White solid, $74 \mathrm{mg}, 90 \%$ yield, $91 \%$ ee. $[\alpha]^{20}{ }_{\mathrm{D}}=-27.10\left(\mathrm{c} 1.0, \mathrm{CHCl}_{3}\right) .{ }^{1} \mathrm{H} \mathrm{NMR}$ $\left(300 \mathrm{MHz}, \mathrm{CDCl}_{3}\right) \delta$ 7.83-7.65 (m, 5H), 7.62-7.57 (m, 1H), 7.49-7.37 (m, 2H), 7.37-7.10 (m, 8H), $4.95(\mathrm{t}, J=7.3 \mathrm{~Hz}, 1 \mathrm{H}), 3.81(\mathrm{~d}, J=7.4 \mathrm{~Hz}, 2 \mathrm{H}) \cdot{ }^{13} \mathrm{C}$ NMR $\left(125 \mathrm{MHz}, \mathrm{CDCl}_{3}\right) \delta 177.8,152.8,141.8,139.5,136.2,132.7,131.6,128.1,127.9$, $127.3,127.2,127.1,126.9,126.3,125.7,125.6,125.21,125.18,125.0,118.5$, 115.8, 47.2, 40.5. HRMS (ESI): $\mathrm{m} / \mathrm{z}[\mathrm{M}-\mathrm{H}]^{-}$calcd for $\mathrm{C}_{25} \mathrm{H}_{18} \mathrm{NO}_{3} \mathrm{~S}: 412.1007$; found: 412.1011 .

HPLC: Chiralpak AD-H column $(250 \mathrm{~mm})$; detected at $224 \mathrm{~nm}$; hexane $/ i$-propanol $=85 / 15$; flow $=0.7$ $\mathrm{mL} / \mathrm{min}$; Retention time: $23.1 \mathrm{~min}$ (major), $27.4 \mathrm{~min} .91 \%$ ee.

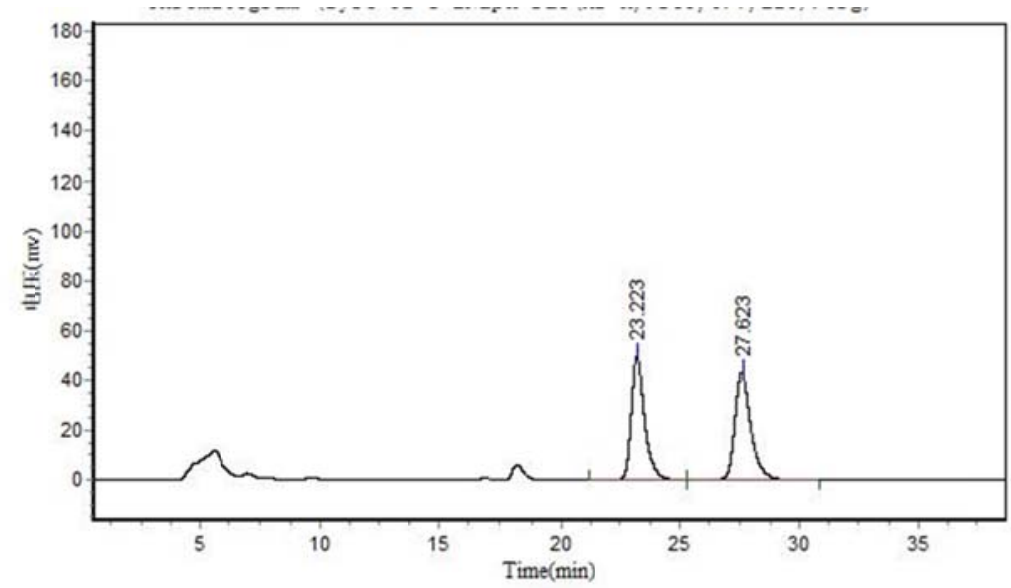

Results

\begin{tabular}{|c|c|c|c|c|c|}
\hline \\
\hline Peak No. & Peak ID & Ret Time & Height & Area & Conc. \\
\hline 1 & & 23.223 & 49836.664 & 1921951.750 & 48.8094 \\
\hline 2 & & 27.623 & 43333.070 & 2015719.000 & 51.1906 \\
\hline Total & & & 93169.734 & 3937670.750 & 100.0000 \\
\hline
\end{tabular}

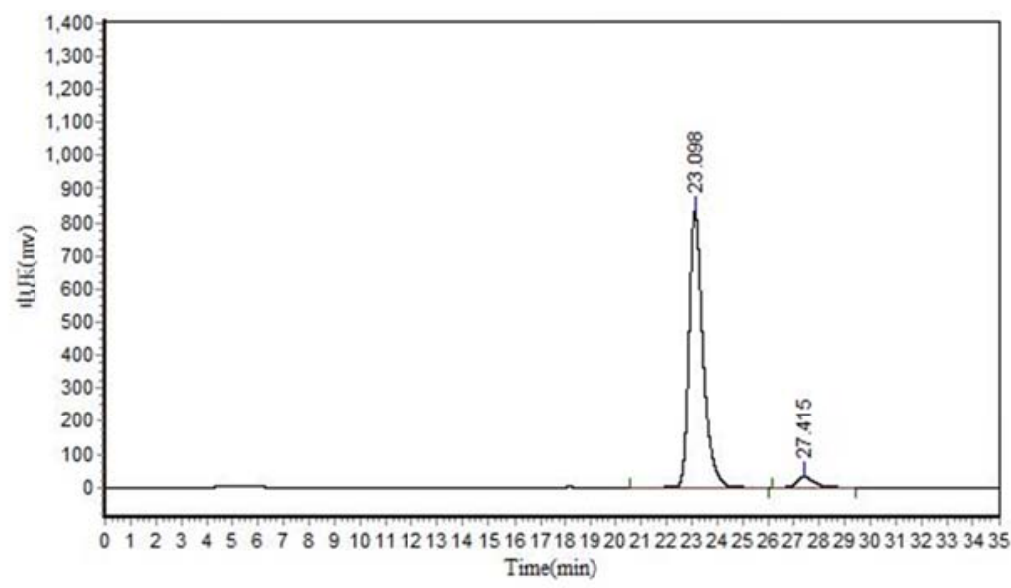

\begin{tabular}{|c|c|c|c|c|c|}
\hline \multicolumn{6}{|c|}{ Results } \\
\hline Peak No. & Peak ID & Ret Time & Height & Area & Conc. \\
\hline$\overline{1}$ & & 23.098 & 836899.563 & 31708012.000 & 95.5370 \\
\hline 2 & & 27.415 & 31651.045 & 1481233.625 & 4.4630 \\
\hline Total & & & $\$ 6 \$ 550.607$ & 33189245.625 & 100.0000 \\
\hline
\end{tabular}


(R)-4-(2-phenyl-2-(m-tolyl)ethyl)benzo[e][1,2,3]oxathiazine 2,2-dioxide (3i)

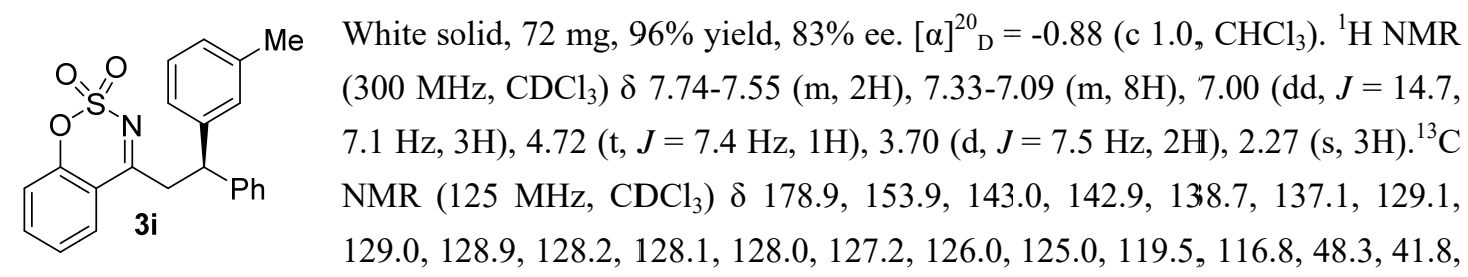

21.8. HRMS (ESI): $m / z$ [M-H] calcd for $\mathrm{C}_{22} \mathrm{H}_{18} \mathrm{NO}_{3} \mathrm{~S}: 376.1007$; found: 376.1002 .

HPLC: Chiralpak AD-H column $(250 \mathrm{~mm})$; detected at $224 \mathrm{~nm}$; hexane $/$ - propanol $=95 / 5$; flow $=0.7$ $\mathrm{mL} / \mathrm{min}$; Retention time: $24.5 \mathrm{~min}$ (major), $26.6 \mathrm{~min}$. 83\% ee.
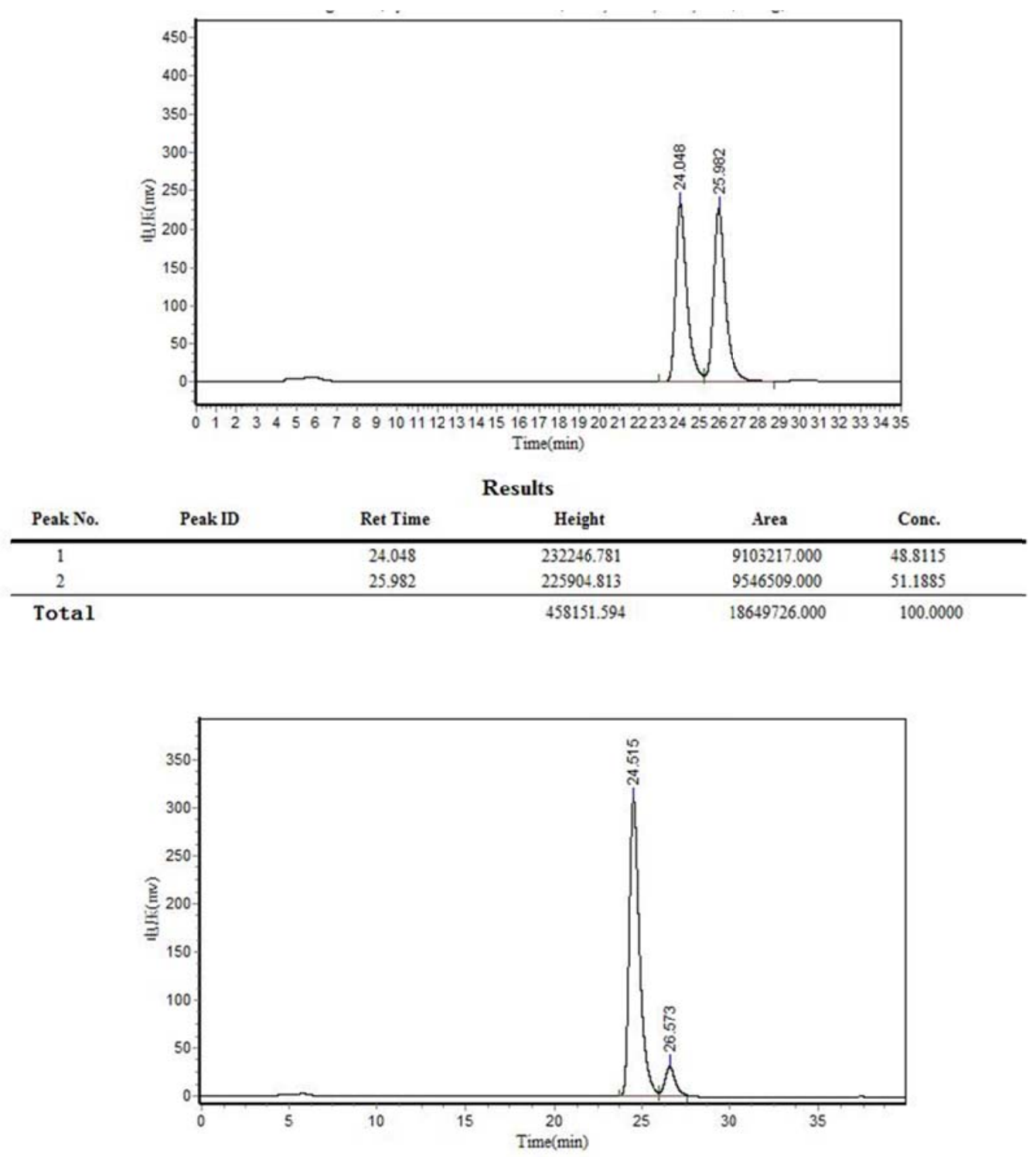

Results

\begin{tabular}{cccccc}
\multirow{2}{*}{ Peak No. } & Peak ID & Ret Time & Height & Area & Conc. \\
\hline 1 & 24.515 & 309803.656 & 12679226.000 & 91.2983 \\
2 & 26.573 & 30261.352 & 1208466.000 & 8.7017 \\
\hline Total & & 340065.008 & 13887692.000 & 100.0000
\end{tabular}


(R)-3-ethyl-3-(3-methoxyphenyl)-2,3-dihydrobenzo[d]isothiazole 1,1-dioxide (3j)<smiles>COc1cccc(C(CC2=NS(=O)(=O)Oc3ccccc32)c2ccccc2)c1</smiles>

White solid, $72 \mathrm{mg}, 92 \%$ yield, $84 \%$ ee. $[\alpha]^{20}{ }_{\mathrm{D}}=5.03\left(\mathrm{c} 1.0, \mathrm{CHCl}_{3}\right) .{ }^{1} \mathrm{H} \mathrm{NMR}$ $\left(300 \mathrm{MHz}, \mathrm{CDCl}_{3}\right) \delta 7.68(\mathrm{dd}, J=17.4,7.8 \mathrm{~Hz}, 2 \mathrm{H}), 7.44-7.08(\mathrm{~m}, 8 \mathrm{H})$, 6.94-6.63 (m, 3H), 4.77 (t, $J=7.4 \mathrm{~Hz}, 1 \mathrm{H}), 3.75$ (s, 3H), 3.73 (d, $J=7.6 \mathrm{~Hz}$, 2H). ${ }^{13} \mathrm{C}$ NMR $\left(125 \mathrm{MHz}, \mathrm{CDCl}_{3}\right) \delta 178.4,159.8,153.5,144.3,142.5,136.8$,

$129.7,128.7,127.9,127.7,126.9,125.7,120.1,119.2,116.5,113.9,112.0$, 55.2, 47.9, 41.3. HRMS (ESI): $\mathrm{m} / \mathrm{z}$ [M-H] ${ }^{-}$calcd for $\mathrm{C}_{22} \mathrm{H}_{18} \mathrm{NO}_{4} \mathrm{~S}: 392.0957$; found: 392.0958 .

HPLC: Chiralpak IC column $(250 \mathrm{~mm})$; detected at $224 \mathrm{~nm}$; hexane/i-propanol $=85 / 15$; flow $=0.7$ $\mathrm{mL} / \mathrm{min}$; Retention time: $50.3 \mathrm{~min}, 75.5 \mathrm{~min}$ (major). 84\% ee.

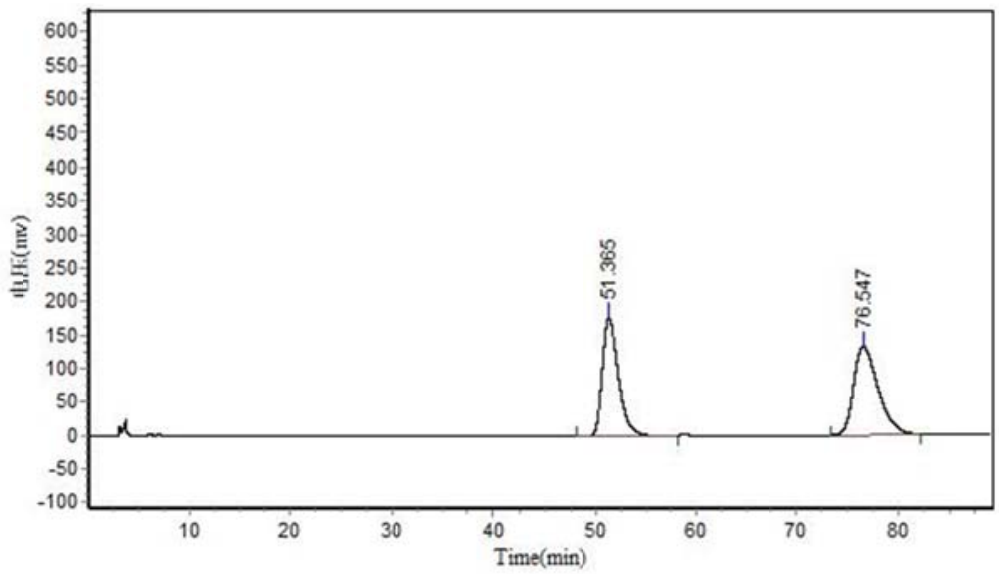

\begin{tabular}{|c|c|c|c|c|c|}
\hline \multicolumn{6}{|c|}{ Results } \\
\hline Peak No. & Peak ID & Ret Time & Height & Area & Conc. \\
\hline 1 & & 51.365 & 176085.078 & 19357770.000 & 46.5915 \\
\hline 2 & & 76.547 & 132754.188 & 22190062.000 & 53.4085 \\
\hline Total & & & $30 \$ 839.266$ & 41547832.000 & 100.0000 \\
\hline
\end{tabular}

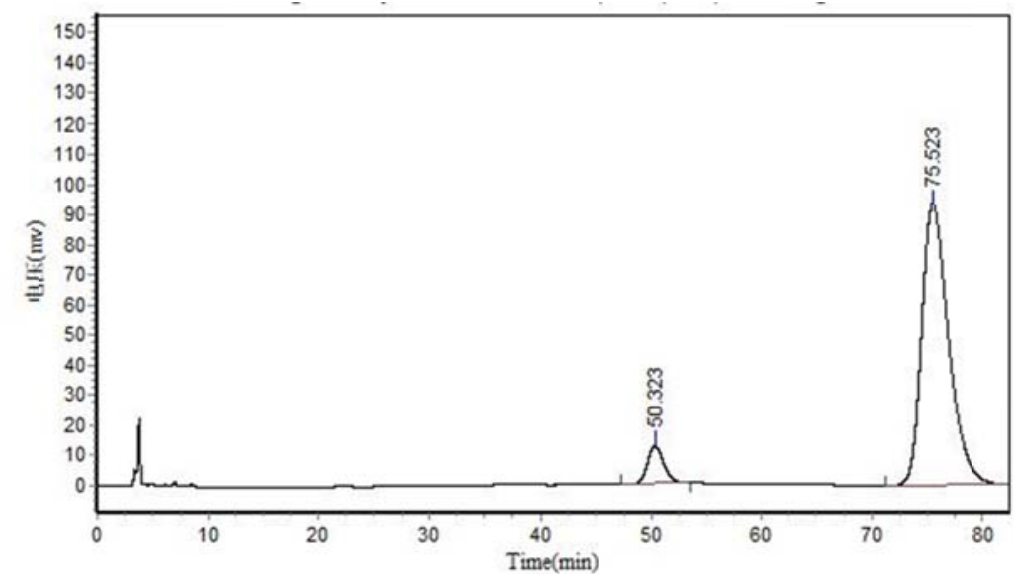

\begin{tabular}{|c|c|c|c|c|c|}
\hline \multicolumn{6}{|c|}{ Results } \\
\hline Peak No. & Peak ID & Ret Time & Height & Area & Conc. \\
\hline 1 & & 50.323 & 12856.569 & 1416722.375 & 8.2130 \\
\hline 2 & & 75.523 & 93597.570 & 15833040.000 & 91.7870 \\
\hline Total & & & 106454.140 & 17249762.375 & 100.0000 \\
\hline
\end{tabular}


(R)-4-(2-(3,4-dimethoxyphenyl)-2-phenylethyl)benzo[e][1,2,3]oxathiazine 2,2-dioxide (3k)

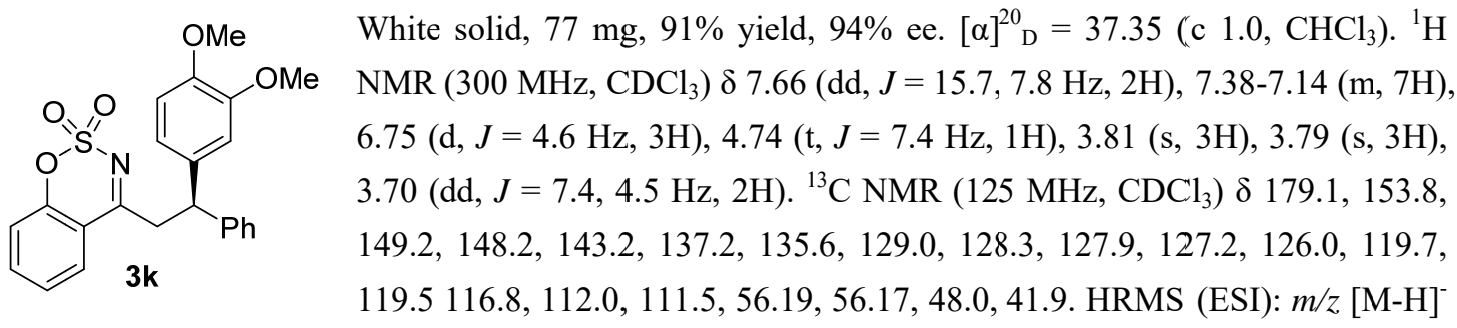
calcd for $\mathrm{C}_{23} \mathrm{H}_{20} \mathrm{NO}_{5} \mathrm{~S}: 422.1062$; found: 422.1060 .

HPLC: Chiralpak AD-H column $(250 \mathrm{~mm})$; detected at $224 \mathrm{~nm}$; hexane/i-propanol $=80 / 20$; flow $=0.7$ $\mathrm{mL} / \mathrm{min}$; Retention time: $20.4 \mathrm{~min}$ (major), $30.0 \mathrm{~min}$. 94\% ee.

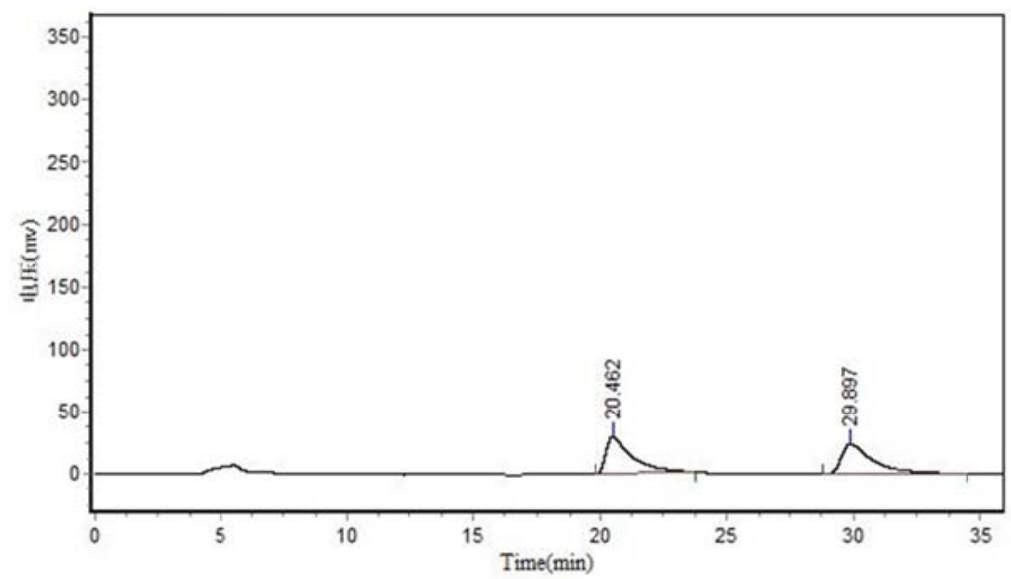

\begin{tabular}{|c|c|c|c|c|c|}
\hline \multicolumn{6}{|c|}{ Results } \\
\hline Peak No. & Peak ID & Ret Time & Height & Area & Conc. \\
\hline 1 & & 20.462 & 30091.244 & 2175865.000 & 50.1845 \\
\hline 2 & & 29.897 & 24537.277 & 2159866.000 & 49.8155 \\
\hline Total & & & 54628.521 & 4335731.000 & 100.0000 \\
\hline
\end{tabular}

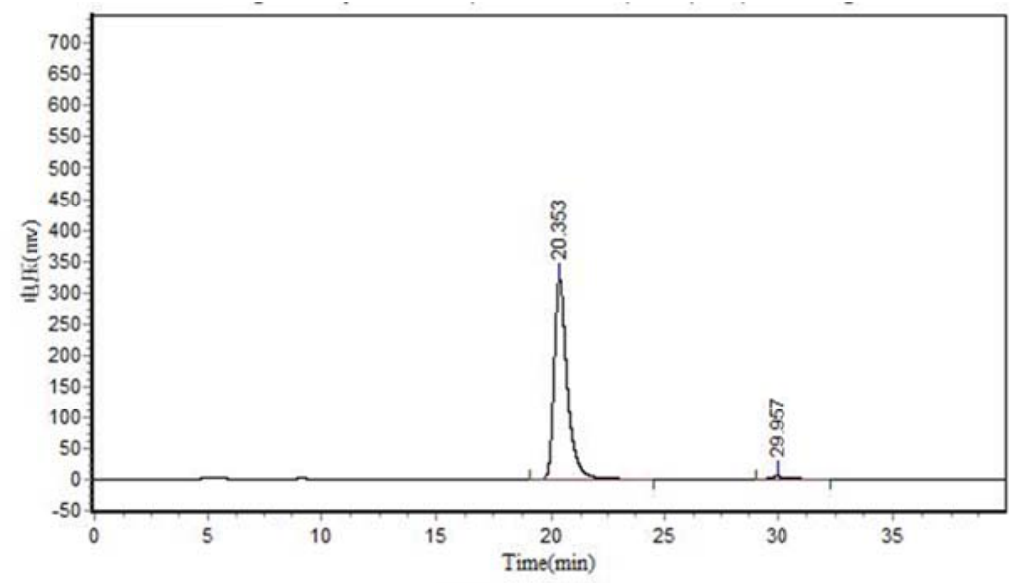

Results

\begin{tabular}{|c|c|c|c|c|c|}
\hline \multicolumn{6}{|c|}{ sec } \\
\hline Peak No. & Peak ID & Ret Time & Height & Area & Conc. \\
\hline 1 & & 20.353 & 324807.250 & 13278501.000 & 97.0347 \\
\hline 2 & & 29.957 & 5115.366 & 405784.500 & 2.9653 \\
\hline Total & & & 329922.616 & 13684285.500 & 100.0000 \\
\hline
\end{tabular}


(R)-6-methyl-4-(2-(naphthalen-1-yl)-2-phenylethyl)benzo[e][1,2,3]oxathiazine 2,2-dioxide (3I)<smiles></smiles>

White solid, $84 \mathrm{mg}, 98 \%$ yield, $98 \%$ ee. $[\alpha]^{20}{ }_{\mathrm{D}}=-73.93\left(\mathrm{c} 1.0, \mathrm{CHCl}_{3}\right) .{ }^{1} \mathrm{H}$ NMR (300 MHz, $\left.\mathrm{CDCl}_{3}\right) \delta$ 8.19-7.99 (m, 1H), 7.90-7.63 (m, 2H), 7.51-7.35 (m, $5 \mathrm{H}), 7.34-7.19(\mathrm{~m}, 5 \mathrm{H}), 7.12(\mathrm{dd}, J=18.9,7.6 \mathrm{~Hz}, 2 \mathrm{H}), 5.56(\mathrm{t}, J=6.0,1 \mathrm{H})$, 3.92-3.75 (m, 2H), $2.28(\mathrm{~s}, 3 \mathrm{H}) .{ }^{13} \mathrm{C} \mathrm{NMR}\left(125 \mathrm{MHz}, \mathrm{CDCl}_{3}\right) \delta$ 179.2, 151.8, $142.91138 .8,137.9,136.0,134.5,131.7,129.2,129.1,128.2,128.1,127.2$, $126.8,126.1,125.5,124.8,124.0,119.1,116.7,44.3,41.9,21.1$. HRMS (ESI): $m / z$ [M-H] ${ }^{-}$calcd for $\mathrm{C}_{26} \mathrm{H}_{21} \mathrm{NO}_{3} \mathrm{~S}: 426.1169$; found: 426.1175 .

HPLC: Chiralpak AD-H column $(250 \mathrm{~mm})$; detected at $224 \mathrm{~nm}$; hexane $/ \mathrm{i}$-propanol $=95 / 5$; flow $=1.0$ $\mathrm{mL} / \mathrm{min}$; Retention time: $18.6 \mathrm{~min}$ (major), $22.8 \mathrm{~min} .98 \%$ ee.

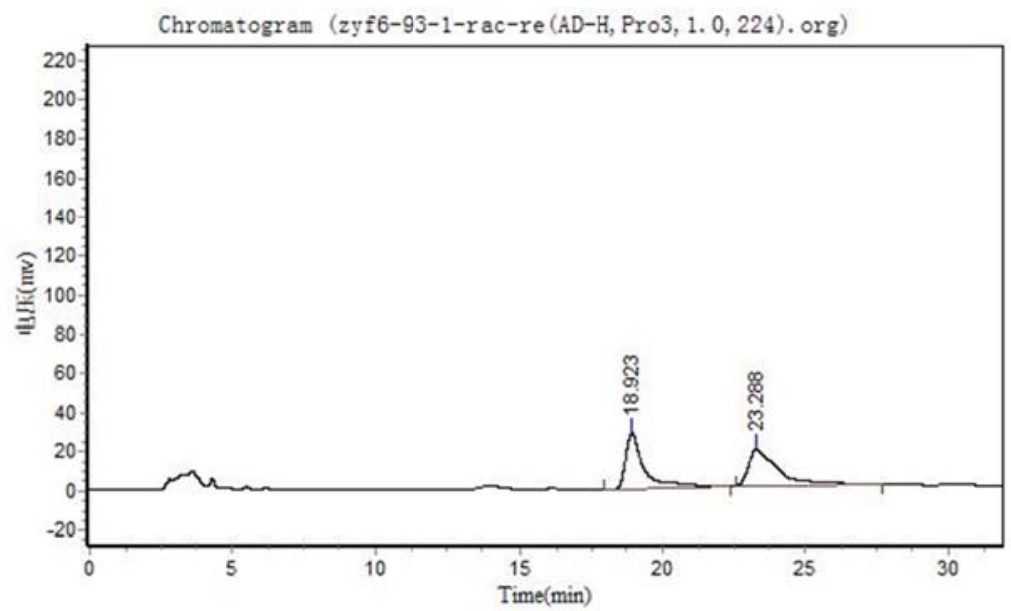

\begin{tabular}{|c|c|c|c|c|c|}
\hline \multicolumn{6}{|c|}{ Results } \\
\hline Peak No. & Peak ID & Ret Time & Height & Area & Conc. \\
\hline 1 & & 18.923 & 28634.553 & 1330819.500 & 50.0564 \\
\hline 2 & & 23.288 & 18530.742 & 1327819.375 & 49.9436 \\
\hline Total & & & 47165.295 & 2658638.875 & 100.0000 \\
\hline
\end{tabular}

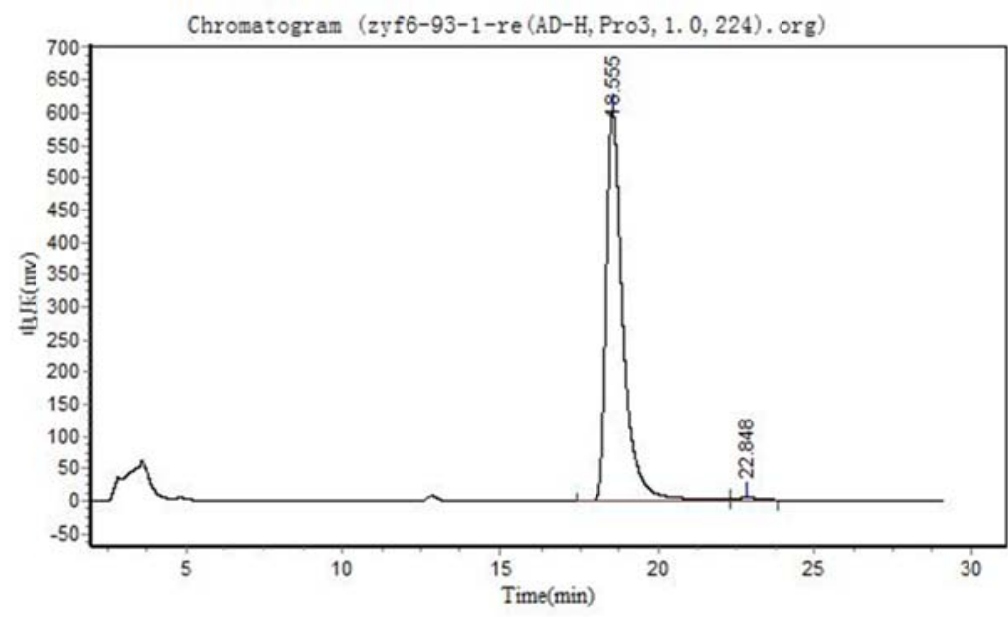

Results

\begin{tabular}{cccccc} 
Peak No. & Peak ID & Ret Time & Height & Area & Conc. \\
\hline 1 & 18.555 & 604682.500 & 22641412.000 & 99.0199 \\
2 & 22.848 & 4533.357 & 224100.891 & 0.9801 \\
\hline Total & & 609215.857 & 22865512.891 & 100.0000
\end{tabular}


(R)-6-methyl-4-(2-phenyl-2-(p-tolyl)ethyl)benzo[e][1,2,3]oxathiazine 2,2-dioxide (3m)

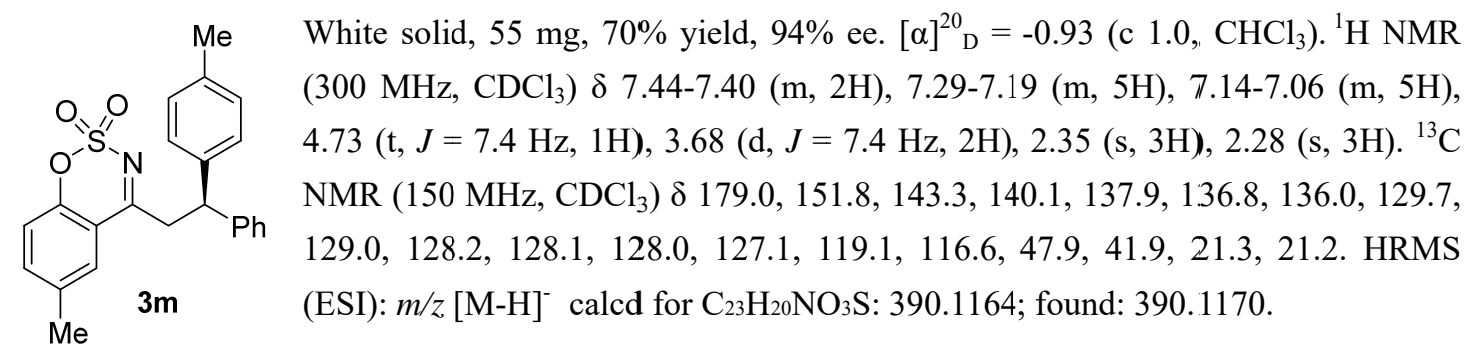

HPLC: Chiralpak IC column $(250 \mathrm{~mm})$; detected at $224 \mathrm{~nm}$; hexane $/$ - -propanol $=85 / 15$; flow $=1.0$ $\mathrm{mL} / \mathrm{min}$; Retention time: $29.7 \mathrm{~min}$ (major), $33.8 \mathrm{~min} .94 \%$ ee.

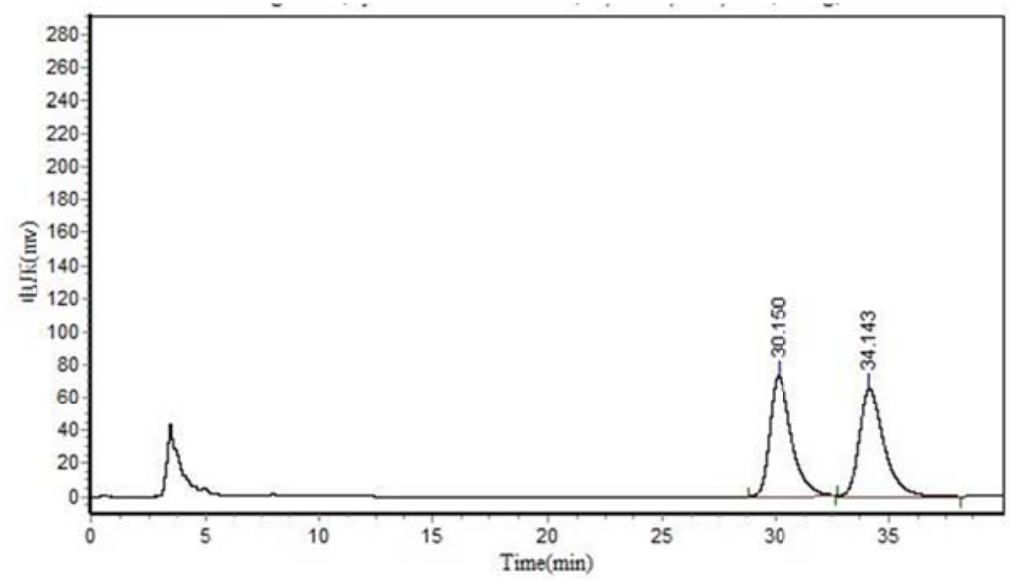

\begin{tabular}{|c|c|c|c|c|c|}
\hline \multicolumn{6}{|c|}{ Results } \\
\hline Peak No. & Peak ID & Ret Time & Height & Area & Conc. \\
\hline 1 & & 30.150 & 73130.422 & 4835304.500 & 49.7323 \\
\hline 2 & & 34.143 & 65600.586 & 4887352.000 & 50.2677 \\
\hline Total & & & 138731.008 & 9722656.500 & 100.0000 \\
\hline
\end{tabular}

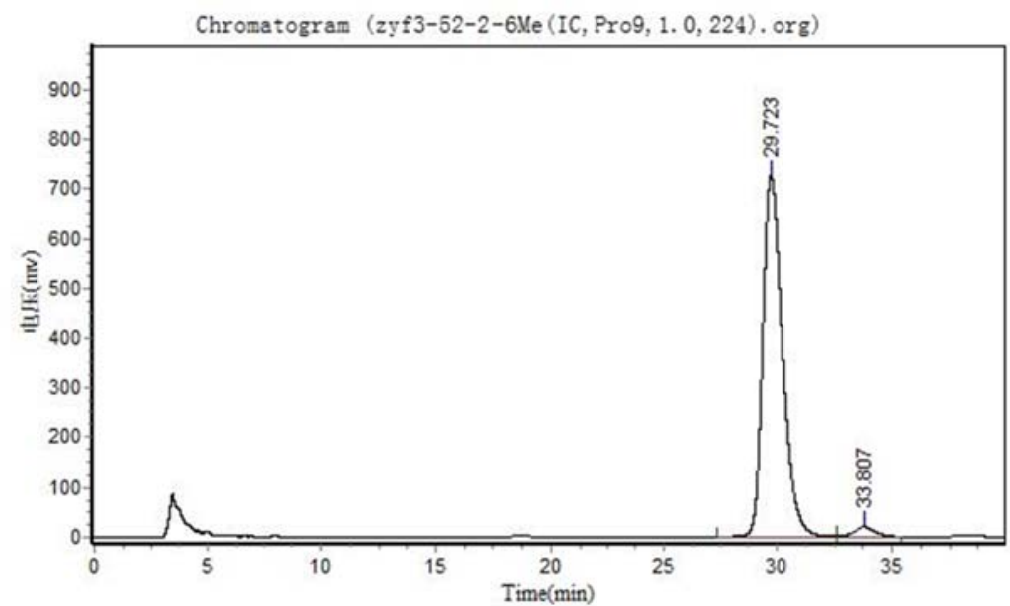

Results

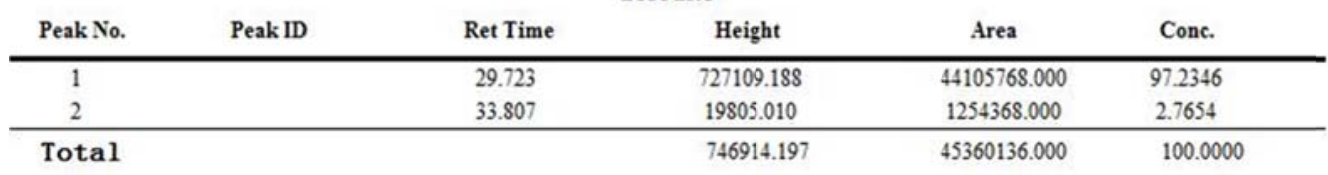


(R)-6-chloro-4-(2-phenyl-2-(p-tolyl)ethyl)benzo[e][1,2,3]oxathiazine 2,2-dioxide (3n)

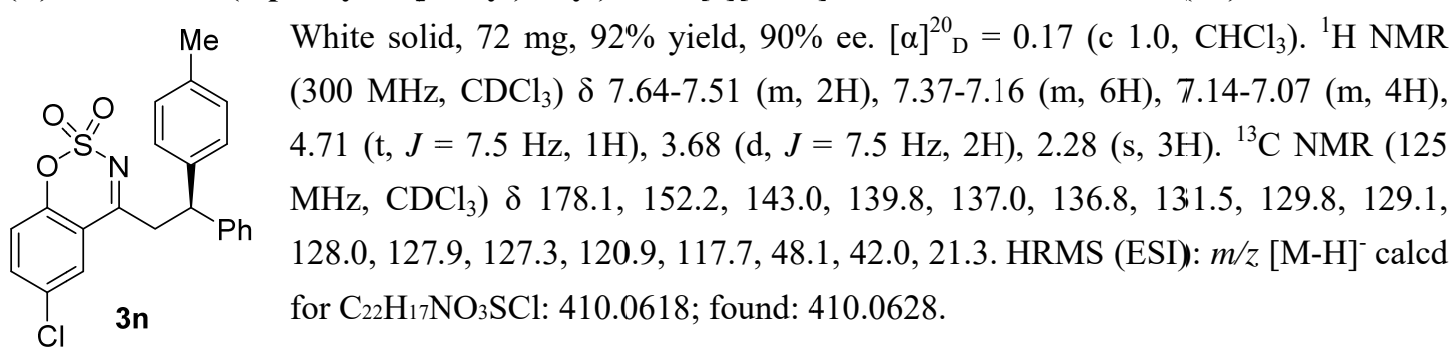

HPLC: Chiralpak IC column $(250 \mathrm{~mm})$; detected at $224 \mathrm{~nm}$; hexane/i-propanol $=85 / 15$; flow $=0.7$ $\mathrm{mL} / \mathrm{min}$; Retention time: $27.2 \mathrm{~min}$ (major), $32.8 \mathrm{~min}$. 90\% ee.

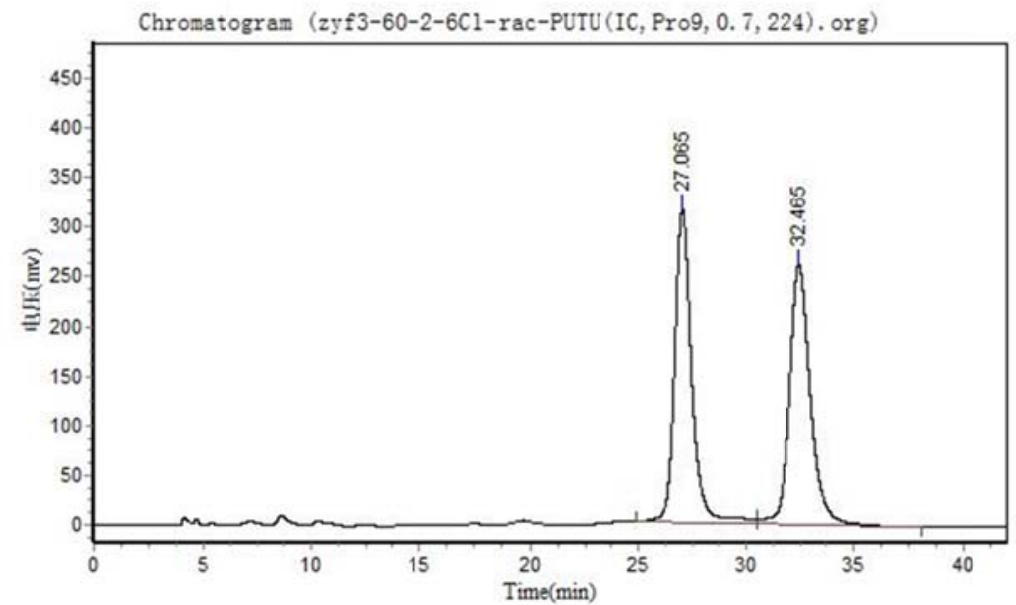

\begin{tabular}{cccccc} 
& \multicolumn{2}{c}{ Results } & Area & Conc. \\
\hline 1 & Peak ID & Ret Time & Height & 18241284.000 & 50.3502 \\
2 & 27.065 & 317049.813 & 17987572.000 & 49.6498 \\
\hline Total & 32.465 & 262296.656 & 36228856.000 & 100.0000
\end{tabular}

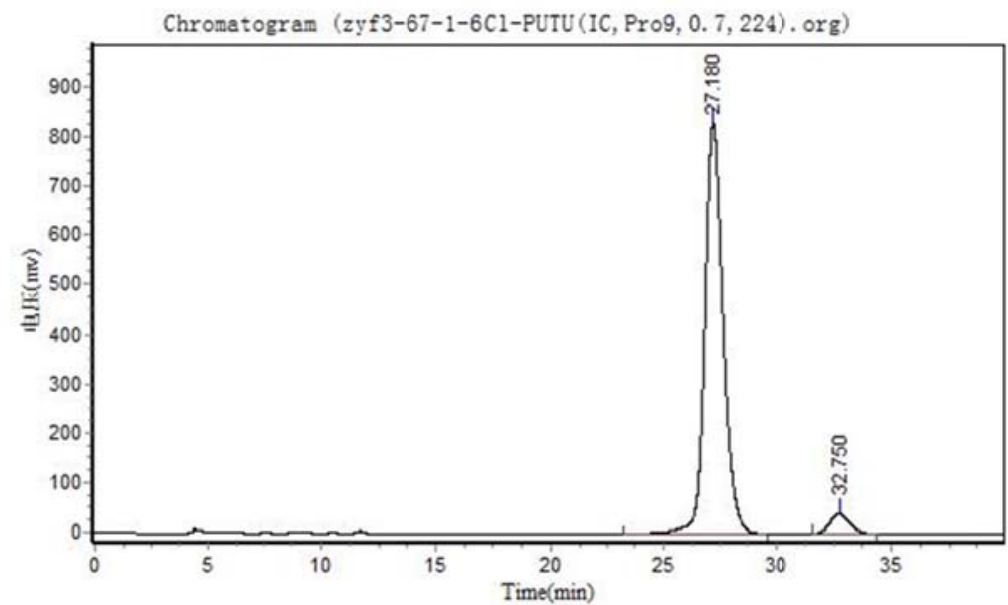

\begin{tabular}{|c|c|c|c|c|c|}
\hline \multicolumn{6}{|c|}{ Results } \\
\hline Peak No. & Peak ID & Ret Time & Height & Area & Conc. \\
\hline 1 & & 27.180 & 829627.063 & 46530608.000 & 94.8557 \\
\hline 2 & & 32.750 & 41268.141 & 2523497.250 & 5.1443 \\
\hline Total & & & 870895.203 & 49054105.250 & 100.0000 \\
\hline
\end{tabular}


(S)-4-(2-(3-chlorophenyl)-2-phenylethyl)benzo[e][1,2,3]oxathiazine 2,2-dioxide (3o)

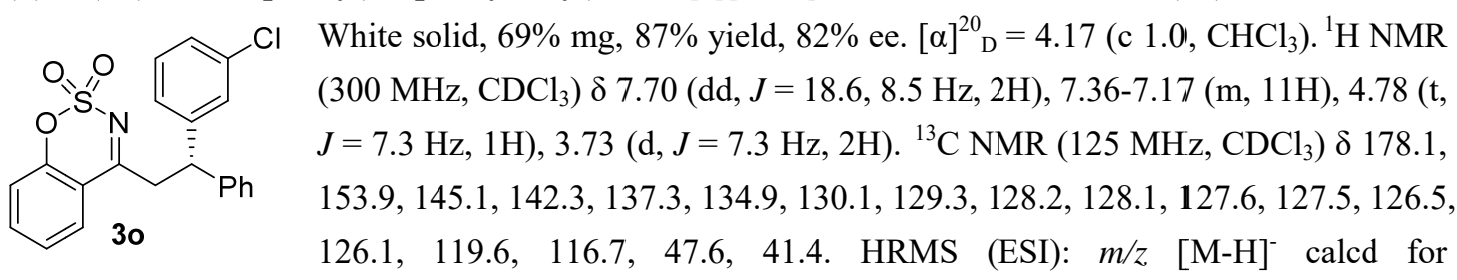
$\mathrm{C}_{21} \mathrm{H}_{15} \mathrm{NO}_{3} \mathrm{SCl}$ : 396.0461 ; found: 396.0465 .

HPLC: Chiralpak IC column $(250 \mathrm{~mm})$; detected at $224 \mathrm{~nm}$; hexane $/$ - -propanol $=85 / 15$; flow $=0.8$ $\mathrm{mL} / \mathrm{min}$; Retention time: $33.2 \mathrm{~min}, 36.0 \mathrm{~min}$ (major). $82 \%$ ee.

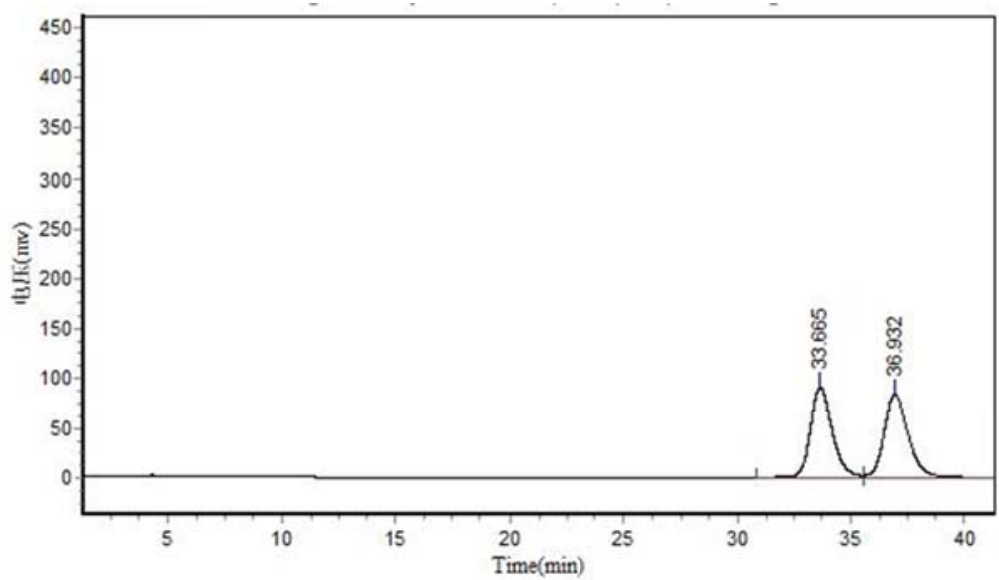

\begin{tabular}{cccccc} 
& \multicolumn{2}{c}{ Results } & Area & Conc. \\
\hline Peak No. & Peak ID & Ret Time & Height & 6011124.000 & 49.7445 \\
2 & 33.665 & 90881.609 & 6072874.500 & 50.2555 \\
\hline Total & 36.932 & 83556.750 & 12083998.500 & 100.0000
\end{tabular}

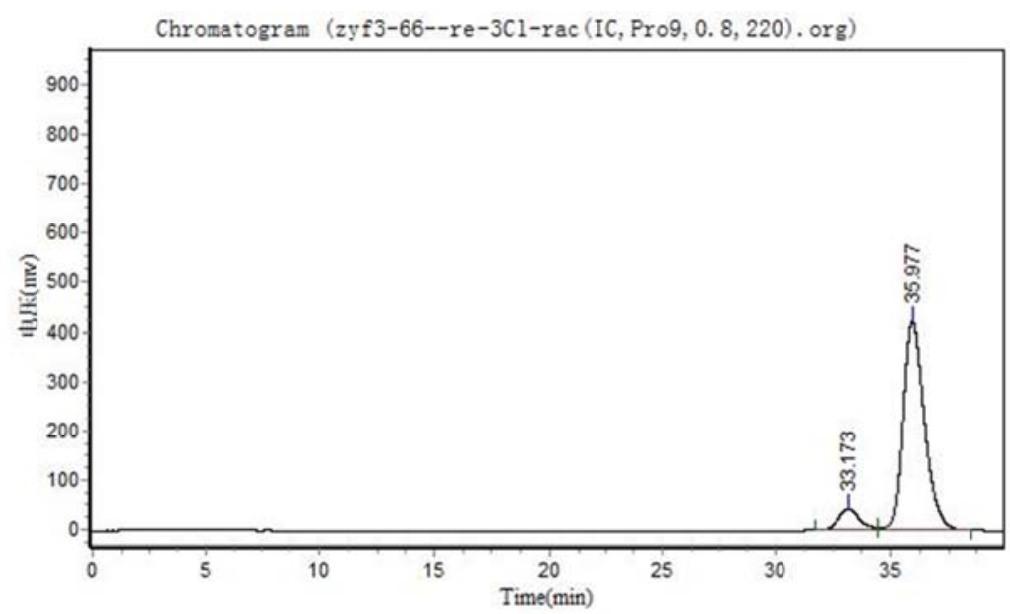

Results

\begin{tabular}{|c|c|c|c|c|c|}
\hline \multicolumn{6}{|c|}{ Kesuits } \\
\hline Peak No. & Peak ID & Ret Time & Height & Area & Conc. \\
\hline 1 & & 33.173 & 43813.879 & 2810313.250 & 9.2494 \\
\hline 2 & & 35.977 & 422232.563 & 27573428.000 & 90.7506 \\
\hline Total & & & 466046.441 & 30383741.250 & 100.0000 \\
\hline
\end{tabular}


(S)-4-(2-phenyl-2-(m-tolyl)ethyl)benzo[e][1,2,3]oxathiazine 2,2-dioxide (3i)<smiles>Cc1cccc(C(CC2=NS(=O)(=O)Oc3ccccc32)c2ccccc2)c1</smiles>

White solid, $75 \mathrm{mg}, 99 \%$ yield, $98 \%$ ee. $[\alpha]^{20}{ }_{\mathrm{D}}=-0.92\left(\mathrm{c} 1.0, \mathrm{CHCl}_{3}\right) .{ }^{1} \mathrm{H} \mathrm{NMR}$ $\left(300 \mathrm{MHz}, \mathrm{CDCl}_{3}\right) \delta$ 7.76-7.57 (m, 2H), 7.36-7.11 (m, 8H), 7.01 (dd, $J=13.1$, $6.9 \mathrm{~Hz}, 3 \mathrm{H}), 4.73(\mathrm{t}, J=7.4 \mathrm{~Hz}, 1 \mathrm{H}), 3.71(\mathrm{~d}, J=7.5 \mathrm{~Hz}, 2 \mathrm{H}), 2.28(\mathrm{~s}, 3 \mathrm{H}) .{ }^{13} \mathrm{C}$ NMR $\left(125 \mathrm{MHz}, \mathrm{CDCl}_{3}\right) \delta 178.9,153.8,143.0,142.9,138.7,137.1,128.3$, 128.1, 128.0, 127.2, 126.0, 125.0, 119.5, 116.8, 48.3, 41.8, 21.8. HRMS (ESI): $\mathrm{m} / \mathrm{z}$ [M-H] $]^{-}$calcd for $\mathrm{C}_{22} \mathrm{H}_{18} \mathrm{NO}_{3} \mathrm{~S}$ : 376.1013; found: 376.1006 .

HPLC: Chiralpak AD-H column $(250 \mathrm{~mm})$; detected at $224 \mathrm{~nm}$; hexane/i-propanol = 95/5; flow $=0.7$ $\mathrm{mL} / \mathrm{min}$; Retention time: $24.4 \mathrm{~min}, 26.3 \mathrm{~min}$ (major). $88 \%$ ee.

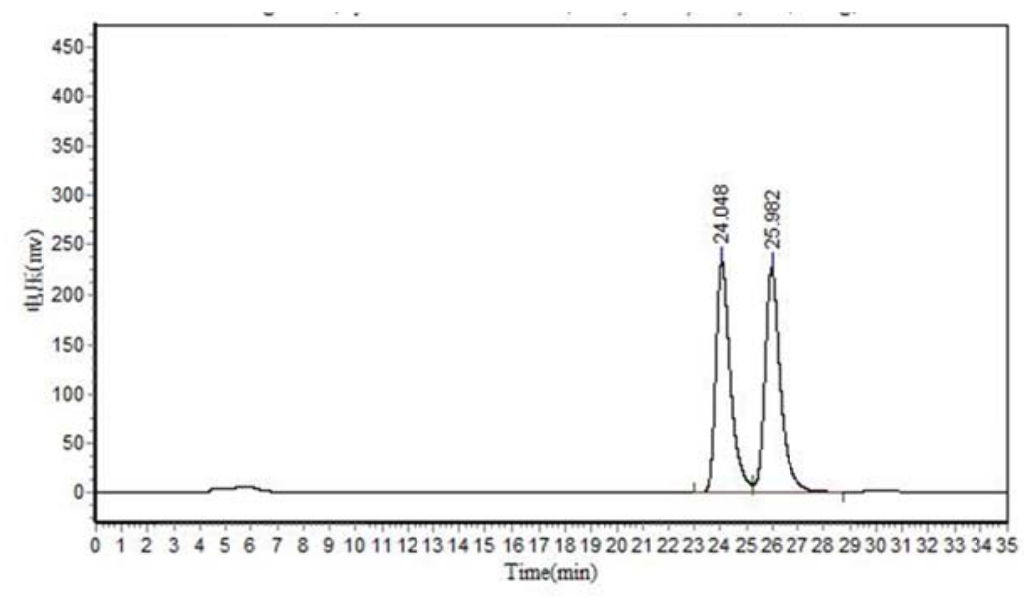

\begin{tabular}{cccccc} 
& & \multicolumn{2}{c}{ Results } & Area & Conc. \\
\hline 1 & Peak ID & Ret Time & Height & 9103217.000 & 48.8115 \\
2 & 24.048 & 232246.781 & 9546509.000 & 51.1885 \\
\hline Total & 25.982 & 225904.813 & 18649726.000 & 100.0000
\end{tabular}

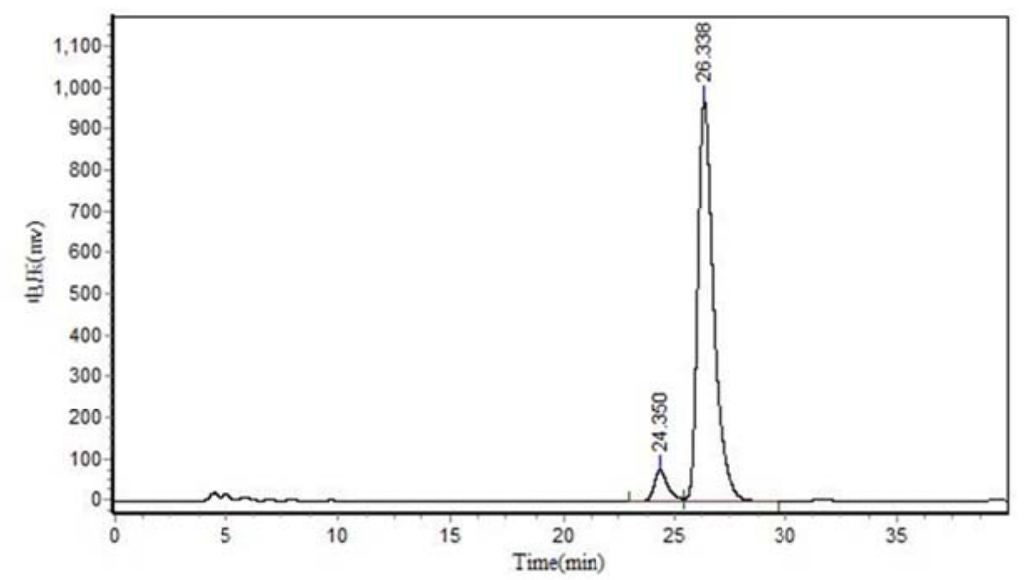

\begin{tabular}{|c|c|c|c|c|c|}
\hline \multicolumn{6}{|c|}{ Results } \\
\hline Peak No. & Peak ID & Ret Time & Height & Area & Conc. \\
\hline 1 & & 24.350 & 74105.430 & 3181472.750 & 6.2337 \\
\hline 2 & & 26.338 & 970357.375 & 47854952.000 & 93.7663 \\
\hline Total & & & 1044462.805 & 51036424.750 & 100.0000 \\
\hline
\end{tabular}




\section{(S)-4-(2-phenyl-2-(p-tolyl)ethyl)benzo[e][1,2,3]oxathiazine 2,2-dioxide (3a')}

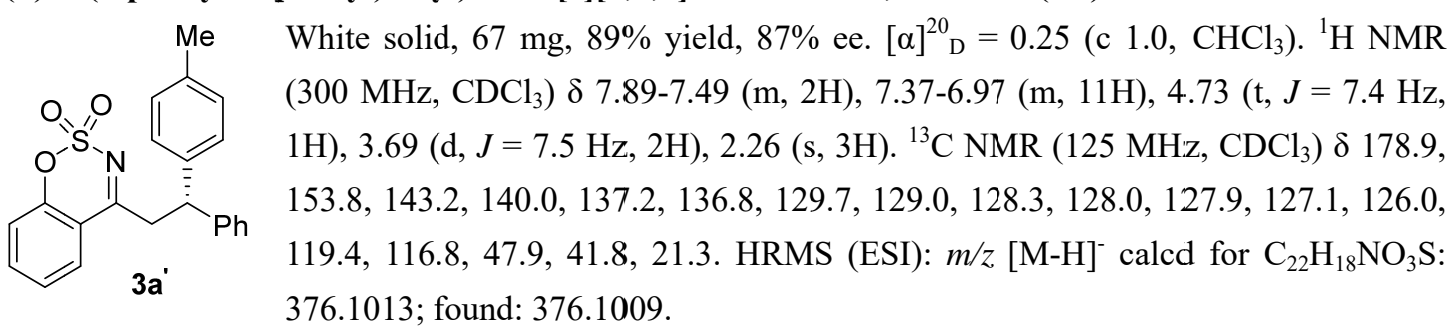

HPLC: Chiralpak IC column $(250 \mathrm{~mm})$; detected at $224 \mathrm{~nm}$; hexane $/$ - -propanol $=80 / 20$; flow $=0.7$ $\mathrm{mL} / \mathrm{min}$; Retention time: $25.6 \mathrm{~min}, 28.8 \mathrm{~min}$ (major). $87 \%$ ee.

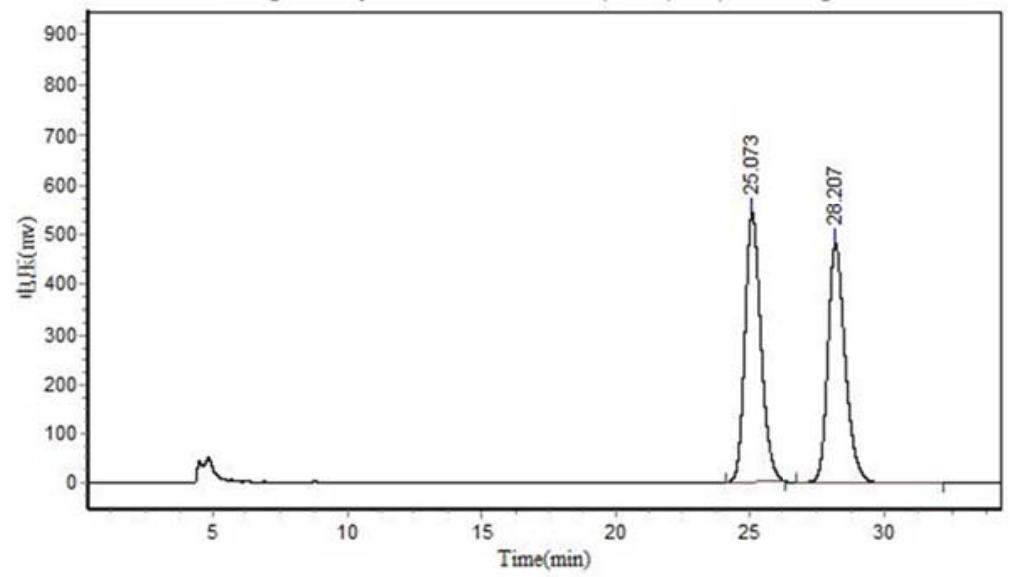

\begin{tabular}{|c|c|c|c|c|c|}
\hline \multicolumn{6}{|c|}{ Results } \\
\hline Peak No. & Peak ID & Ret Time & Height & Area & Conc. \\
\hline 1 & & 25.073 & 541697.438 & 22517524.000 & 50.7869 \\
\hline 2 & & 28.207 & 482062.719 & 21819736.000 & 49.2131 \\
\hline Total & & & 1023760.156 & 44337260.000 & 100.0000 \\
\hline
\end{tabular}

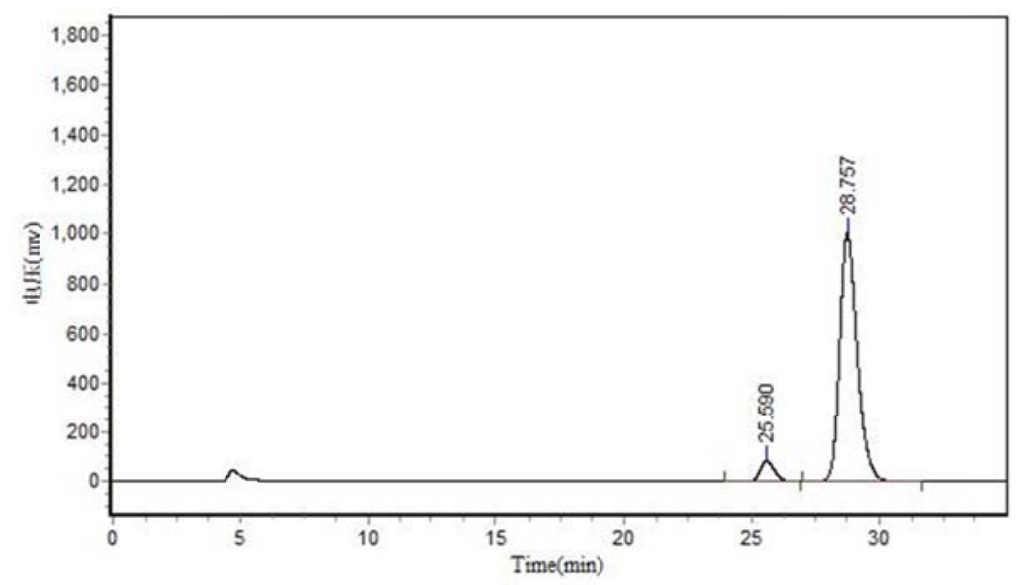

\begin{tabular}{|c|c|c|c|c|c|}
\hline \multicolumn{6}{|c|}{ Results } \\
\hline Peak No. & Peak ID & Ret Time & Height & Area & Conc. \\
\hline$\frac{1}{1}$ & & 25.590 & 83165.461 & 3279034.750 & 6.4107 \\
\hline 2 & & 28.757 & 1000805.000 & 47869968.000 & 93.5892 \\
\hline Total & & & 1083970.461 & 51149002.750 & 100.0000 \\
\hline
\end{tabular}


(R)-4-(2-(naphthalen-1-yl)-2-(p-tolyl)ethyl)benzo[e][1,2,3]oxathiazine 2,2-dioxide (3p)<smiles>Cc1ccc(C(CC2=NS(=O)(=O)Oc3ccccc32)c2cccc3ccccc23)cc1</smiles>

White solid, $85 \mathrm{mg}, 99 \%$ yield, $99 \%$ ee. $[\alpha]^{20}{ }_{\mathrm{D}}=-69.03\left(\mathrm{c} 1.0, \mathrm{CHCl}_{3}\right) .{ }^{1} \mathrm{H}$ NMR (300 MHz, $\left.\mathrm{CDCl}_{3}\right) \delta$ 8.27-7.96 (m, 1H), 7.89-7.71 (m, 2H), $7.63(\mathrm{dd}$, $J=13.6,7.8 \mathrm{~Hz}, 2 \mathrm{H}), 7.46(\mathrm{~d}, J=4.5 \mathrm{~Hz}, 4 \mathrm{H}), 7.31-7.11(\mathrm{~m}, 4 \mathrm{H}), 7.04$ (d, $J=7.5 \mathrm{~Hz}, 2 \mathrm{H}), 5.56(\mathrm{t}, J=7.1 \mathrm{~Hz}, 1 \mathrm{H}), 3.93-3.75(\mathrm{~m}, 2 \mathrm{H}), 2.25(\mathrm{~s}, 3 \mathrm{H})$. ${ }^{13} \mathrm{C}$ NMR $\left(125 \mathrm{MHz}, \mathrm{CDCl}_{3}\right) \delta 179.1,153.7,139.8,138.9,137.1,136.8$, $134.4,131.6,129.7,129.2,128.2,128.1,128.0,126.7,126.01,125.96,125.5,124.6,123.9,119.4$, 116.8, 43.6, 42.0, 21.3. HRMS (ESI): $\mathrm{m} / \mathrm{z}$ [M-H] ${ }^{-}$calcd for $\mathrm{C}_{26} \mathrm{H}_{21} \mathrm{NO}_{3} \mathrm{~S}: 426.1169$; found: 426.1177.

HPLC: Chiralpak AD-H column $(250 \mathrm{~mm})$; detected at $224 \mathrm{~nm}$; hexane $/ \mathrm{i}$-propanol = 95/5; flow = 1.0 $\mathrm{mL} / \mathrm{min}$; Retention time: $21.4 \mathrm{~min}$ (major), $26.2 \mathrm{~min} .99 \%$ ee.

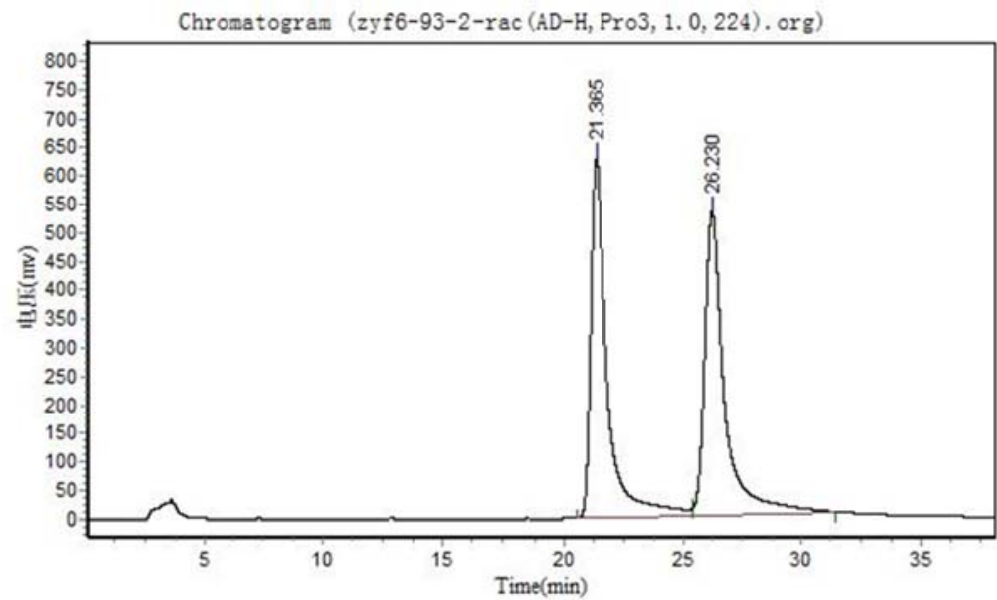

\begin{tabular}{|c|c|c|c|c|c|}
\hline \multicolumn{6}{|c|}{ Results } \\
\hline Peak No. & Peak ID & Ret Time & Height & Area & Conc. \\
\hline 1 & & 21.365 & 629430.375 & 30042450.000 & 49.4979 \\
\hline 2 & & 26.230 & 531109.750 & 30651976.000 & 50.5021 \\
\hline Total & & & 1160540.125 & 60694426.000 & 100.0000 \\
\hline
\end{tabular}

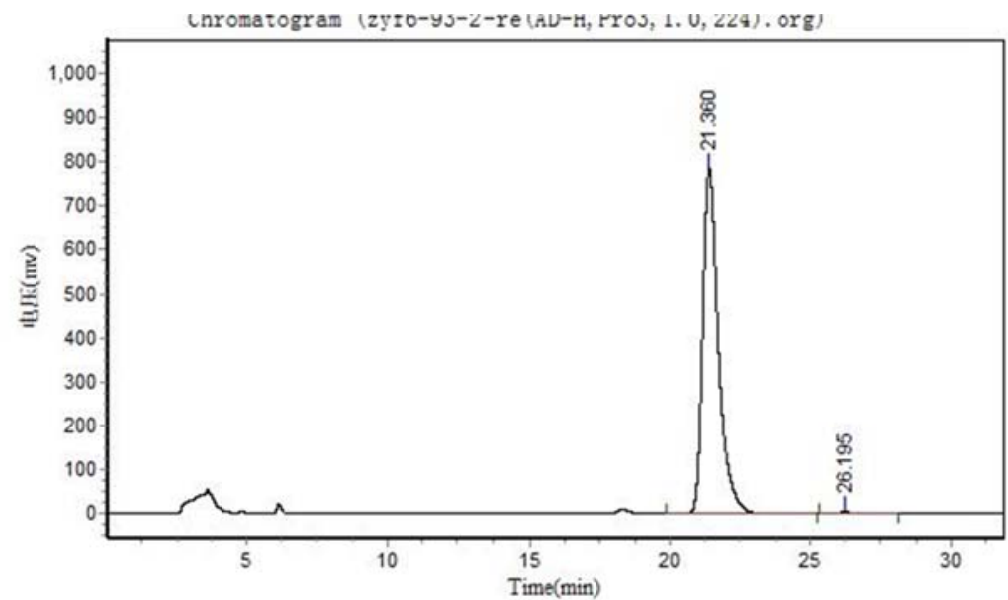

\begin{tabular}{|c|c|c|c|c|c|}
\hline \multicolumn{6}{|c|}{ Results } \\
\hline Peak No. & Peak ID & Ret Time & Height & Area & Conc. \\
\hline 1 & & 21.360 & 784647.563 & 30647918.000 & $99.340 \mathrm{~S}$ \\
\hline 2 & & 26.195 & 4437.576 & 203379.656 & 0.6592 \\
\hline Total & & & 789085.138 & 30851297.656 & 100.0000 \\
\hline
\end{tabular}


(S)-4-(2-(2-fluorophenyl)-2-phenylethyl)benzo[e][1,2,3]oxathiazine 2,2-dioxide (3f')<smiles>O=S1(=O)N=C(CC(c2ccccc2)c2ccccc2F)c2ccccc2O1</smiles>

White solid, $71 \mathrm{mg}, 93 \%$ yield, $86 \%$ ee. $[\alpha]^{20}{ }_{\mathrm{D}}=8.65\left(\mathrm{c} 1.0, \mathrm{CHCl}_{3}\right) .{ }^{1} \mathrm{H} \mathrm{NMR}$ $\left(300 \mathrm{MHz}, \mathrm{CDCl}_{3}\right) \delta 7.80(\mathrm{~d}, J=8.0 \mathrm{~Hz}, 1 \mathrm{H}), 7.69-7.64(\mathrm{~m}, 1 \mathrm{H}), 7.39-7.15(\mathrm{~m}$, 9H), 7.13-6.93 (m, 2H), 5.00 (t, $J=7.6 \mathrm{~Hz}, 1 \mathrm{H}), 3.78$ (d, $J=7.6 \mathrm{~Hz}, 2 \mathrm{H}) .{ }^{13} \mathrm{C}$ NMR $\left(125 \mathrm{MHz}, \mathrm{CDCl}_{3}\right) \delta 178.3,160.9\left(\mathrm{~d}, J_{\mathrm{CF}}=243.8 \mathrm{~Hz}\right), 153.9,141.6,137.2$, $129.8\left(\mathrm{~d}, J_{\mathrm{CF}}=13.8 \mathrm{~Hz}\right), 129.5\left(\mathrm{~d}, J_{\mathrm{CF}}=5.0 \mathrm{~Hz}\right), 129.1,129.0,128.14,128.11$, 127.4, 126.1, $124.8\left(\mathrm{~d}, J_{\mathrm{CF}}=3.8 \mathrm{~Hz}\right), 119.6,116.6,116.2\left(\mathrm{~d}, J_{\mathrm{CF}}=21.3 \mathrm{~Hz}\right), 42.3$, 40.6. HRMS (ESI): $\mathrm{m} / \mathrm{z}$ [M-H] $]^{-}$calcd for $\mathrm{C}_{21} \mathrm{H}_{16} \mathrm{NO}_{3} \mathrm{SF}$ : 380.0762 ; found: 380.0753 .

HPLC: Chiralpak IC column $(250 \mathrm{~mm})$; detected at $224 \mathrm{~nm}$; hexane $/ \mathrm{i}$-propanol $=85 / 15$; flow $=0.8$ $\mathrm{mL} / \mathrm{min}$; Retention time: $38.0 \mathrm{~min}, 43.2 \mathrm{~min}$ (major). $86 \%$ ee.

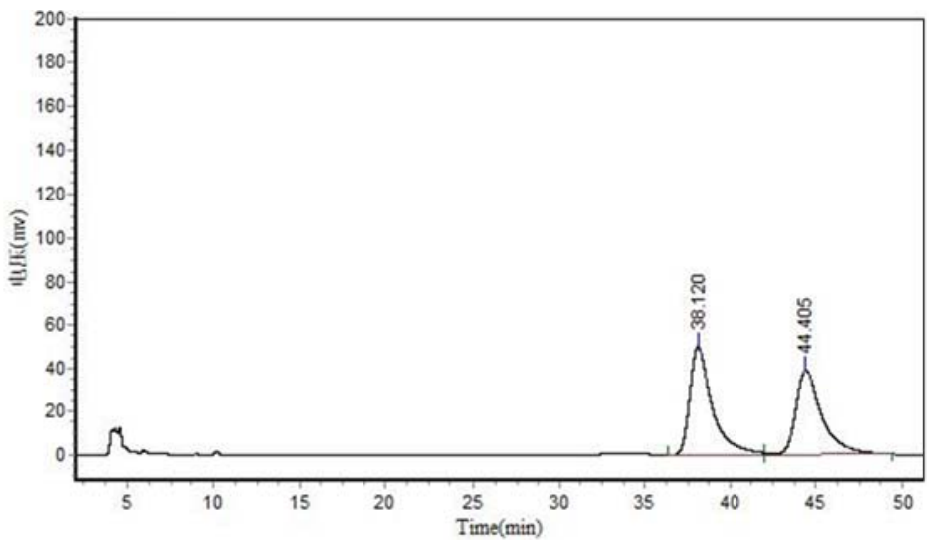

\begin{tabular}{|c|c|c|c|c|c|}
\hline \multicolumn{6}{|c|}{ Results } \\
\hline Peak No. & Peak ID & Ret Time & Height & Area & Conc. \\
\hline 1 & & 38.120 & 49739.223 & 4530980.000 & 52.5626 \\
\hline 2 & & 44.405 & 38704.566 & 4089175.000 & 47.4374 \\
\hline Total & & & $\$ 8443.789$ & $\$ 620155.000$ & $100.000 \mathrm{C}$ \\
\hline
\end{tabular}

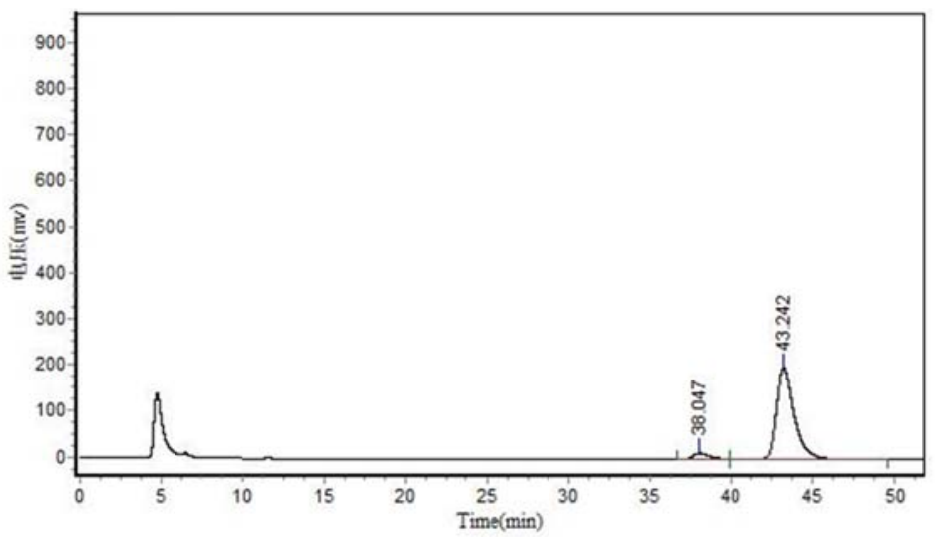

\begin{tabular}{|c|c|c|c|c|c|}
\hline \multicolumn{6}{|c|}{ Results } \\
\hline Peak No. & Peak ID & Ret Time & Height & Area & Conc. \\
\hline 1 & & 38.047 & 14525.836 & 1164728.250 & 6.9373 \\
\hline 2 & & 43.242 & 199867.094 & 15624587.000 & 93.0627 \\
\hline
\end{tabular}


(S)-4-(2-(2-fluorophenyl)-2-(o-tolyl)ethyl)benzo[e][1,2,3]oxathiazine 2,2-dioxide (3q)<smiles>Cc1ccccc1C(CC1=NS(=O)(=O)Oc2ccccc21)c1ccccc1F</smiles>

White solid, $78 \mathrm{mg}, 99 \%$ yield, $99 \%$ ee. $[\alpha]^{20}{ }_{\mathrm{D}}=-72.70\left(\mathrm{c} 1.0, \mathrm{CHCl}_{3}\right) .{ }^{1} \mathrm{H}$ NMR $\left(300 \mathrm{MHz}, \mathrm{CDCl}_{3}\right) \delta 7.79(\mathrm{~d}, J=7.8 \mathrm{~Hz}, 1 \mathrm{H}), 7.70-7.65(\mathrm{~m}, 1 \mathrm{H}), 7.34$ (dd, $J=11.3,7.6 \mathrm{~Hz}, 2 \mathrm{H}), 7.29-6.93(\mathrm{~m}, 8 \mathrm{H}), 5.19(\mathrm{t}, J=7.5 \mathrm{~Hz}, 1 \mathrm{H}), 3.74(\mathrm{~d}$, $J=7.1 \mathrm{~Hz}, 2 \mathrm{H}), 2.26(\mathrm{~s}, 3 \mathrm{H}) .{ }^{13} \mathrm{C} \mathrm{NMR}\left(125 \mathrm{MHz}, \mathrm{CDCl}_{3}\right) \delta 178.4,160.9\left(J_{\mathrm{CF}}\right.$ $=237.5 \mathrm{~Hz}), 153.8,139.4,137.2,136.8,131.3,129.4\left(J_{\mathrm{CF}}=4.1 \mathrm{~Hz}\right), 128.9\left(J_{\mathrm{CF}}\right.$ $=8.3 \mathrm{~Hz}), 128.1,127.4,126.8,126.5,126.1,124.8\left(J_{\mathrm{CF}}=3.3 \mathrm{~Hz}\right), 119.5,116.6,116.0,115.8,41.0$, 37.6, 19.8. HRMS (ESI): $\mathrm{m} / \mathrm{z}$ [M-H] ${ }^{-}$calcd for $\mathrm{C}_{22} \mathrm{H}_{18} \mathrm{NO}_{3} \mathrm{SF}$ : 394.0919; found: 394.0926 .

HPLC: Chiralpak IC column $(250 \mathrm{~mm})$; detected at $224 \mathrm{~nm}$; hexane $/ \mathrm{i}$-propanol $=85 / 15$; flow $=1.0$ $\mathrm{mL} / \mathrm{min}$; Retention time: $19.7 \mathrm{~min}, 21.4$ min (major). 99\% ee.

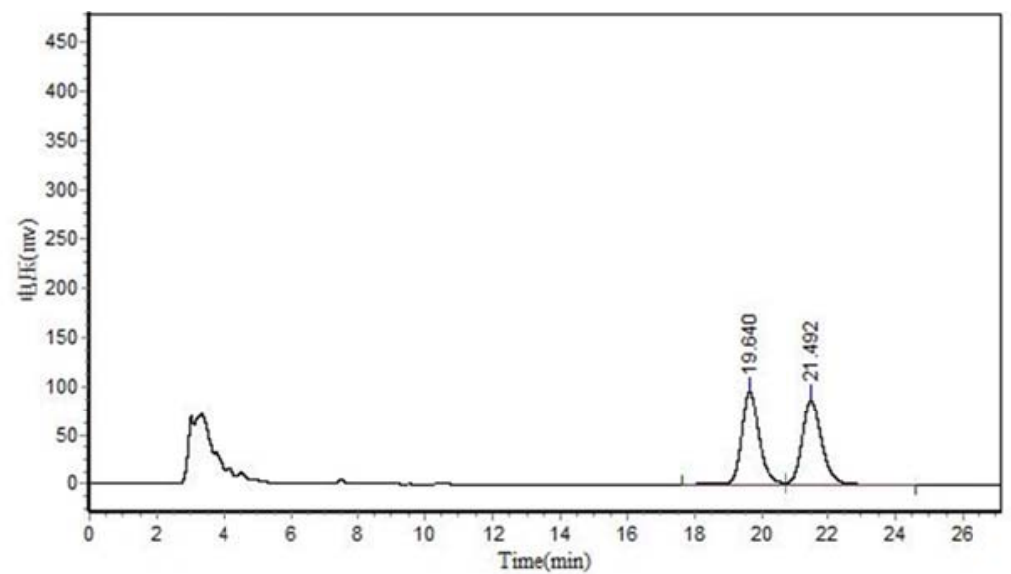

\begin{tabular}{|c|c|c|c|c|c|}
\hline \multicolumn{6}{|c|}{ Results } \\
\hline Peak No. & Peak ID & Ret Time & Height & Area & Conc. \\
\hline 1 & & 19.640 & 94308.219 & 3396961.750 & 49.9548 \\
\hline 2 & & 21.492 & 85673.789 & 3403109.250 & 50.0452 \\
\hline Total & & & 179982.008 & 6800071.000 & 100.0000 \\
\hline
\end{tabular}

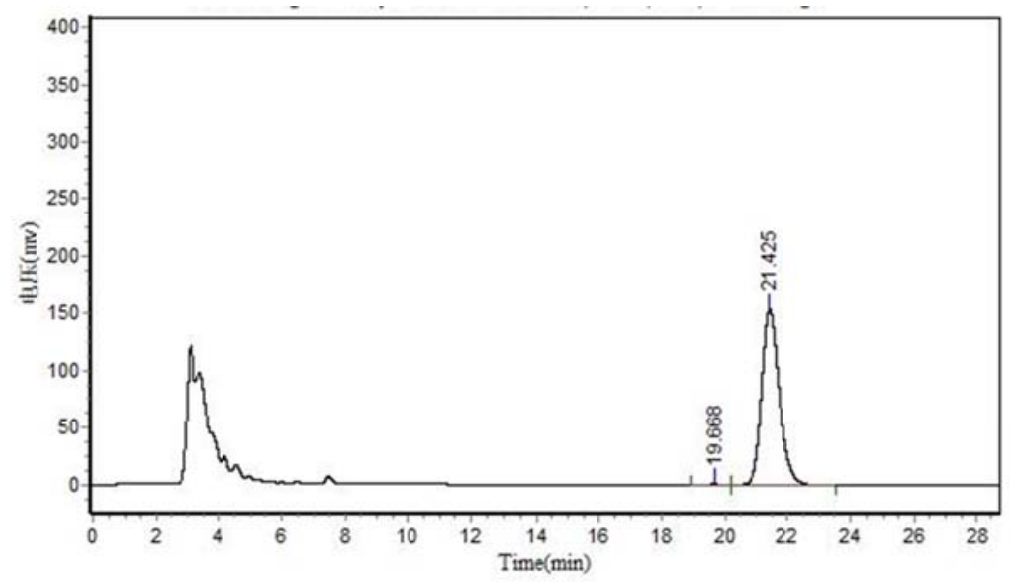

Results

\begin{tabular}{|c|c|c|c|c|c|}
\hline \multicolumn{6}{|c|}{ centa } \\
\hline Peak No. & Peak ID & Ret Time & Height & Area & Conc. \\
\hline 1 & & 19.668 & 1065.443 & 39642.719 & 0.6446 \\
\hline 2 & & 21.425 & 154929.063 & 6110429.500 & 99.3554 \\
\hline Total & & & 155994.506 & 6150072.219 & 100.0000 \\
\hline
\end{tabular}


(R)-4-(4-methoxyphenyl)-4-((R)-2-phenyl-2-(p-tolyl)ethyl)-3,4-dihydrobenzo[e][1,2,3]oxathiazine

\section{2,2-dioxide (4a)}

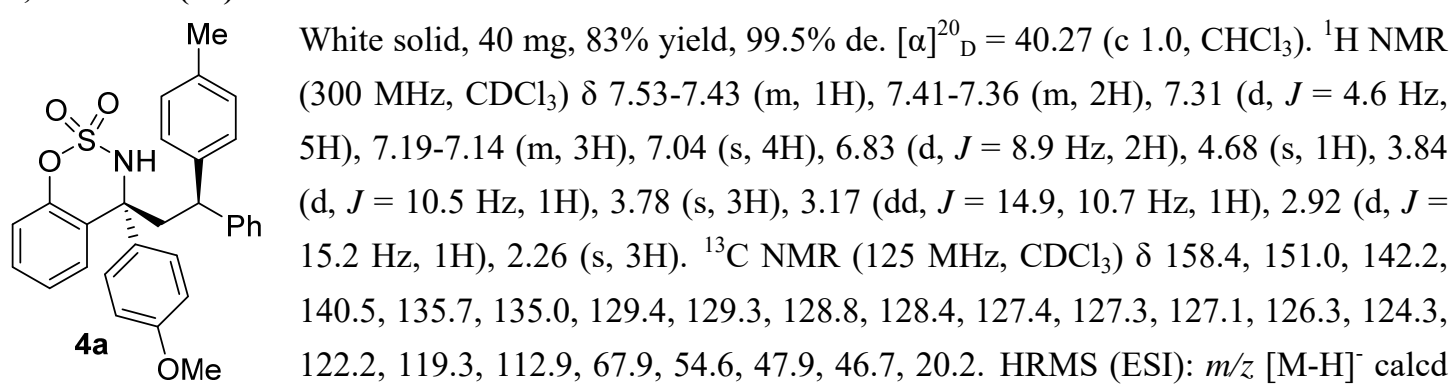
for $\mathrm{C}_{29} \mathrm{H}_{26} \mathrm{NO}_{4} \mathrm{~S}$ : 484.1583 ; found: 484.1585 .

HPLC: Chiralpak IC column $(250 \mathrm{~mm})$; detected at $224 \mathrm{~nm}$; hexane/i-propanol = 70/30; flow $=1.0$ $\mathrm{mL} / \mathrm{min}$; Retention time: $9.4 \mathrm{~min}, 12.3 \mathrm{~min}, 18.7 \mathrm{~min}, 28.6 \mathrm{~min}$ (major). $99.5 \%$ de for $\mathbf{4 a}$.

The reaction of $( \pm)$-3a with $p$-methoxyphenylboronic acid $\mathbf{2} \mathbf{b}$ was conducted under rhodium catalysis with $\mathbf{L} 7(<10 \%$ ee) as ligand to afford 4a. The expected 1,2-addition could take place with concomitant formation of four stereoisomers. The corresponding HPLC spectrum of 4a is shown in Figure 1a.

The reaction of $(R)-\mathbf{3 a}(93 \%$ ee) with $p$-methoxyphenylboronic acid $\mathbf{2 b}$ was conducted under rhodium catalysis with L7 ( $<10 \%$ ee) as ligand. The corresponding HPLC spectrum of $4 \mathbf{a}$ is shown in Figure $1 \mathbf{b}$.

The reaction of $(R)-\mathbf{3 a}(93 \%$ ee) with $p$-methoxyphenylboronic acid $\mathbf{2 b}$ was conducted under rhodium catalysis by using optically pure $\mathbf{L} \mathbf{1}$ as a ligand. The corresponding HPLC spectrum of $\mathbf{4 a}$ is shown in Figure 1c.

The reaction of $(R)-\mathbf{3 a}(93 \%$ ee) with $p$-methoxyphenylboronic acid $\mathbf{2 b}$ was conducted under rhodium catalysis by using optically pure $\mathbf{L} 7$ as a ligand. The corresponding HPLC spectrum of $\mathbf{4 a}$ is shown in Figure 1d. 


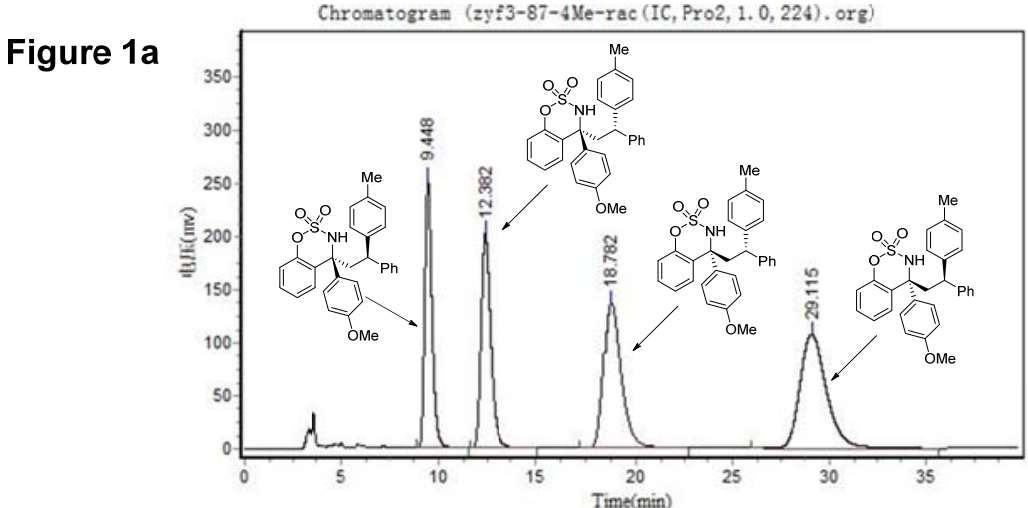

\begin{tabular}{|c|c|c|c|c|c|}
\hline \multicolumn{6}{|c|}{ Results } \\
\hline Peak No. & Peak ID & Ret Time & Height & Area & Conc. \\
\hline 1 & & 9.448 & 252007.578 & 6479004.500 & 20.1947 \\
\hline 2 & & 12.382 & 201910.500 & 7115981.000 & 22.1802 \\
\hline 3 & & 18.782 & 137811.547 & $8368 \$ 45.500$ & 26.0853 \\
\hline 4 & & 29.115 & 107207.484 & 10118790.000 & 31.5398 \\
\hline Total & & & 698937.109 & 32082621.000 & 100.0000 \\
\hline
\end{tabular}

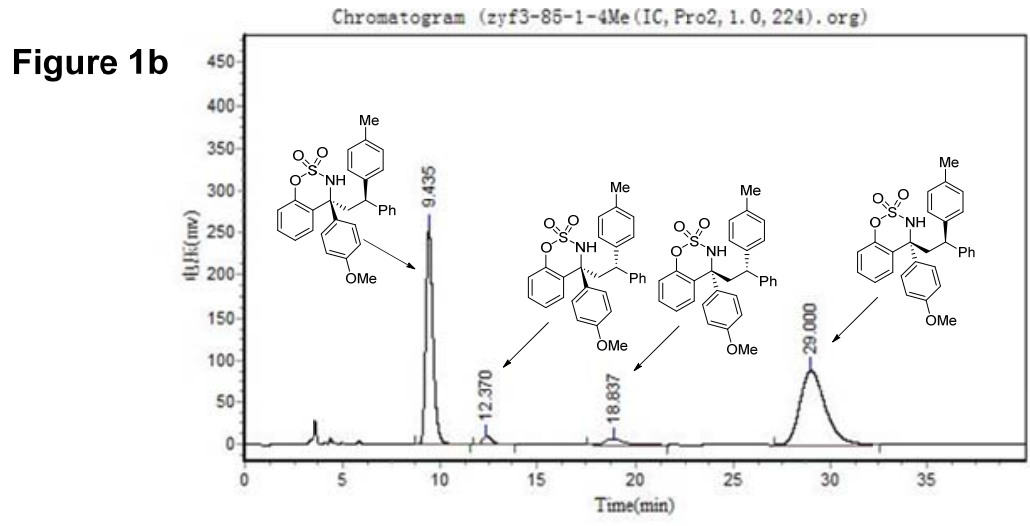

\begin{tabular}{|c|c|c|c|c|c|}
\hline \multicolumn{6}{|c|}{ Results } \\
\hline Peak No. & Peak ID & Ret Time & Height & Area & Conc. \\
\hline 1 & & 9.435 & 255698.797 & 6550177.000 & 42.6581 \\
\hline 2 & & 12.370 & 10119.371 & 356044.500 & 2.3187 \\
\hline 3 & & 18.837 & 7184.857 & 455186.344 & 2.9644 \\
\hline 4 & & 29.000 & $\$ 8427.578$ & 7993659.500 & 52.0588 \\
\hline Total & & & 361430.603 & 15355067,344 & 100.0000 \\
\hline
\end{tabular}

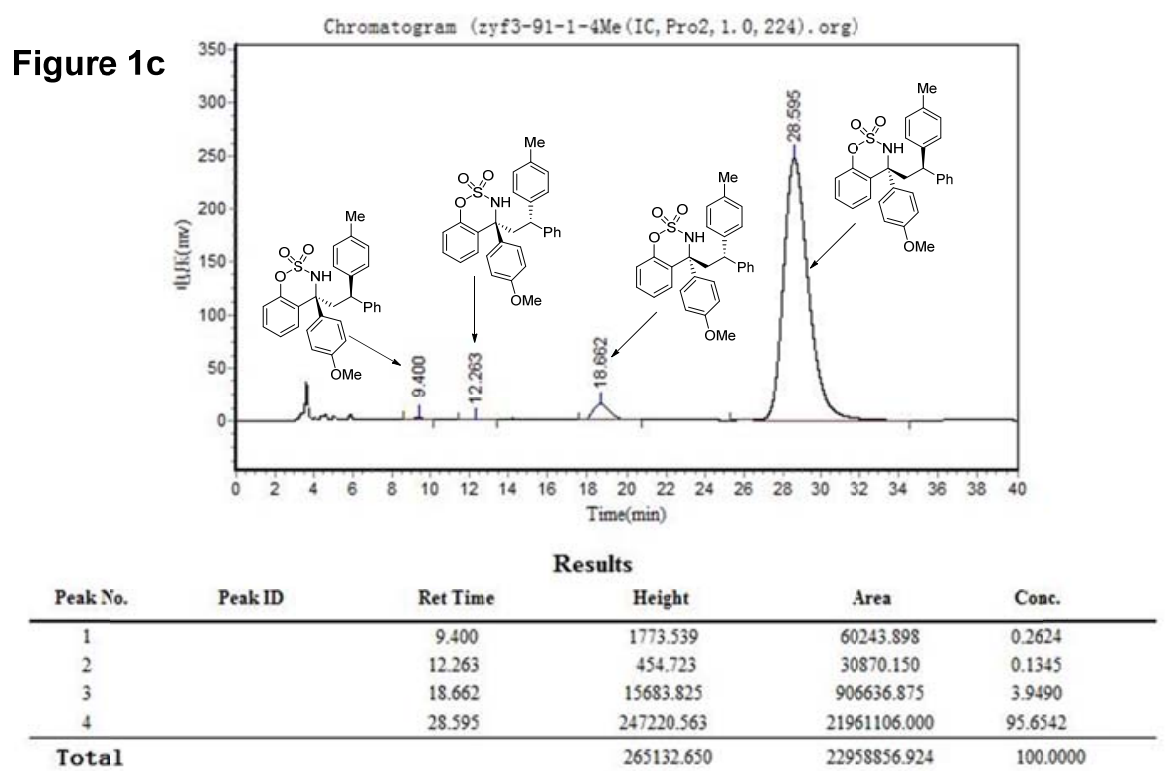



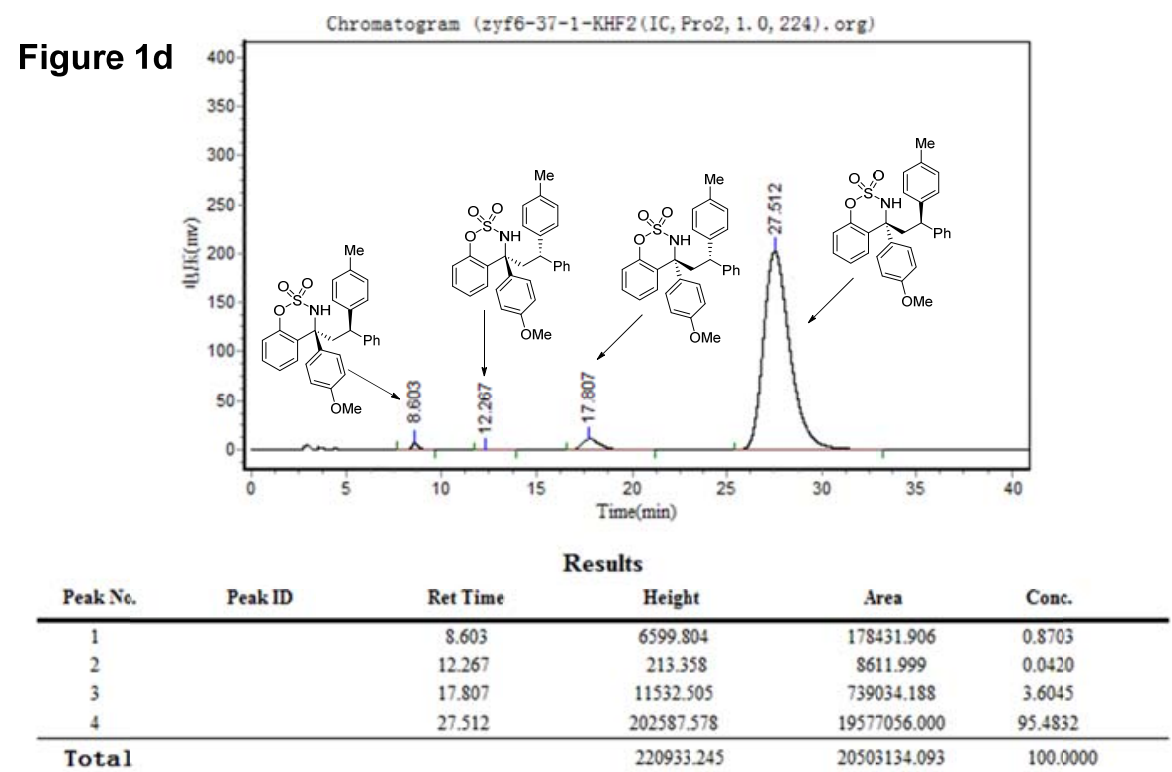

(R)-4-(4-methoxyphenyl)-4-((R)-2-phenyl-2-(o-tolyl)ethyl)-3,4-dihydrobenzo[e][1,2,3]oxathiazine

\section{2,2-dioxide (4b)}<smiles>COc1ccc(C(NC2(c3ccccc3)NS(=O)(=O)Oc3ccccc32)c2ccccc2C)cc1</smiles>

White solid, $33 \mathrm{mg}, 68 \%$ yield, $99 \%$ ee. $[\alpha]^{20}{ }_{\mathrm{D}}=-34.92\left(\mathrm{c} 1.0, \mathrm{CHCl}_{3}\right) .{ }^{1} \mathrm{H}$ NMR $\left(300 \mathrm{MHz}, \mathrm{CDCl}_{3}\right) \delta 7.47(\mathrm{~d}, J=8.0 \mathrm{~Hz}, 2 \mathrm{H}), 7.39-7.34(\mathrm{~m}, 2 \mathrm{H})$, 7.32-6.98 (m, 11H), $6.83(\mathrm{~d}, J=8.8 \mathrm{~Hz}, 2 \mathrm{H}), 4.66(\mathrm{~s}, 1 \mathrm{H}), 4.08(\mathrm{~d}, J=7.6 \mathrm{~Hz}$, $1 \mathrm{H}), 3.78(\mathrm{~s}, 3 \mathrm{H}), 3.23(\mathrm{dd}, J=15.0,10.3 \mathrm{~Hz}, 1 \mathrm{H}), 2.86$ (dd, $J=15.0,2.7 \mathrm{~Hz}$, $1 \mathrm{H}), 1.94(\mathrm{~s}, 3 \mathrm{H}) .{ }^{13} \mathrm{C}$ NMR $\left(125 \mathrm{MHz}, \mathrm{CDCl}_{3}\right) \delta 158.4,151.1,141.7,141.4$, $135.1,134.9,130.1,129.5,129.2,128.5,127.6,127.2,127.0,126.1,125.9$, 125.2, 124.2, 122.2, 119.3, 112.9, 68.1, 54.6, 48.9, 42.6, 18.9. HRMS (ESI): $\mathrm{m} / \mathrm{z}[\mathrm{M}-\mathrm{H}]^{-}$calcd for $\mathrm{C}_{29} \mathrm{H}_{26} \mathrm{NO}_{4} \mathrm{~S}: 484.1583$; found: 484.1587.

HPLC: Chiralpak AD-H column $(250 \mathrm{~mm})$; detected at $224 \mathrm{~nm}$; hexane $/ \mathrm{i}$-propanol $=90 / 10$; flow $=0.7$ $\mathrm{mL} / \mathrm{min}$; Retention time: $10.8 \mathrm{~min}, 13.9$ min (major). 99\% ee.

The reaction of $( \pm)$-3d with $p$-methoxyphenylboronic acid $\mathbf{2 b}$ was conducted under rhodium catalysis by using $\mathbf{L} 7(<10 \%$ ee) as ligand to afford $\mathbf{4 b}$. The expected 1,2-addition could take place with concomitant formation of four stereoisomers. The corresponding HPLC spectrum of $\mathbf{4 b}$ is shown in Figure 2a.

The reaction of $(R)$-3d $(99 \%$ ee) with $p$-methoxyphenylboronic acid $\mathbf{2 b}$ was conducted under rhodium catalysis by using $\mathbf{L} 7(<10 \%$ ee) as a ligand. The corresponding HPLC spectrum is shown in Figure 2b.

The reaction of $(R)$-3d ( $99 \%$ ee) with $p$-methoxyphenylboronic acid $\mathbf{2 b}$ was conducted under rhodium catalysis by using optically pure $\mathbf{L} \mathbf{1}$ as a ligand. The corresponding HPLC spectrum is shown in Figure 2c. 


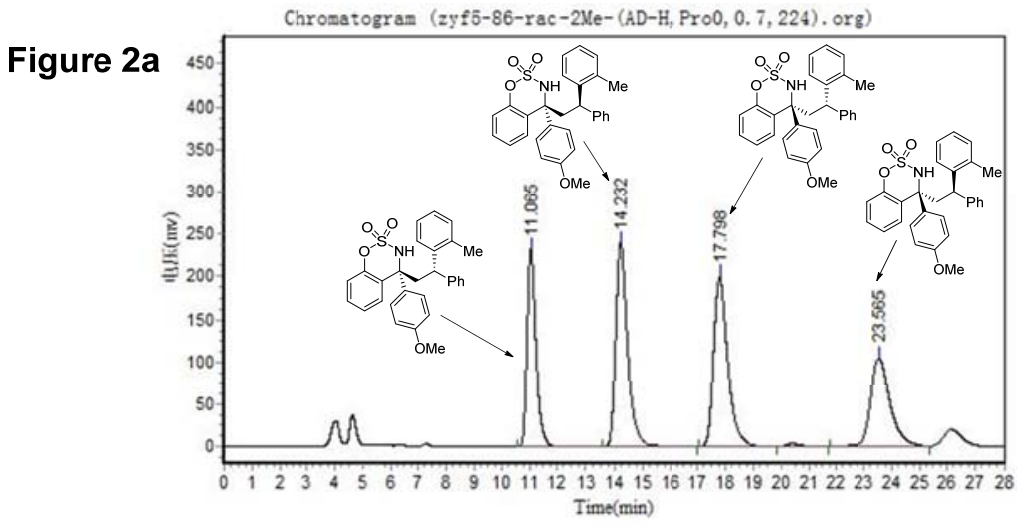

\begin{tabular}{|c|c|c|c|c|c|}
\hline \multicolumn{6}{|c|}{ Results } \\
\hline Peak No. & Peak ID & Ret Time & Height & Area & Conc. \\
\hline 1 & & 11.065 & 232093.625 & 5031964.000 & 20.6251 \\
\hline 2 & & 14.232 & 238679.781 & 7139911.500 & 29.2653 \\
\hline 3 & & 17.798 & 198441.406 & 7068951.500 & 28.9744 \\
\hline 4 & & 23.565 & 104912.781 & 5156401.000 & 21.1352 \\
\hline Total & & & 774127.594 & 24397228.000 & 100.0000 \\
\hline
\end{tabular}

Figure $2 b$

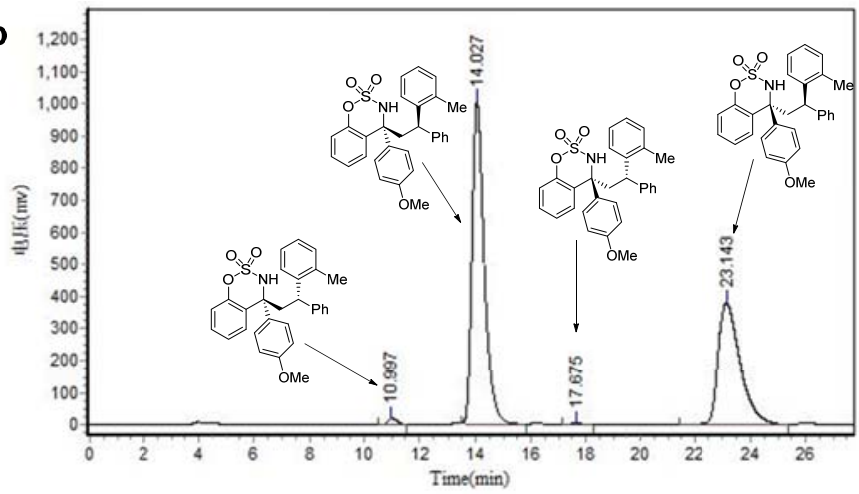

\begin{tabular}{|c|c|c|c|c|c|}
\hline \multicolumn{6}{|c|}{ Results } \\
\hline Peak No. & Peak ID & Ret Time & Height & Area & Conc. \\
\hline 1 & & 10.997 & 21736.084 & 514775.000 & 0.9841 \\
\hline 2 & & 14.027 & 1001987.813 & 30708116.000 & 58.7020 \\
\hline 3 & & 17.675 & 3729.694 & 119321.406 & 0.2281 \\
\hline 4 & & 23.143 & 378754.000 & 20969624.000 & 40.0858 \\
\hline Total & & & 1406207.590 & 52311836.406 & 100.0000 \\
\hline
\end{tabular}

Figure 2c

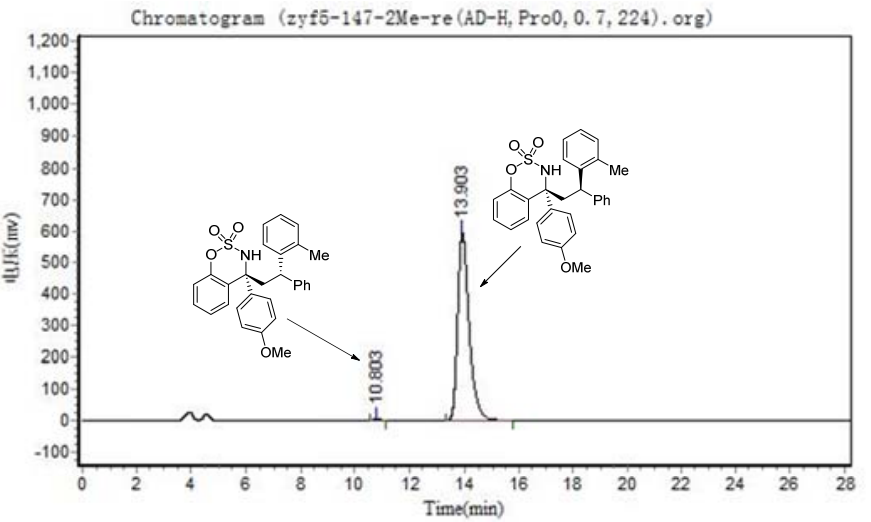

\begin{tabular}{|c|c|c|c|c|c|}
\hline \multicolumn{6}{|c|}{ Results } \\
\hline Peak No. & Peak ID & Ret Time & Height & Area & Conc. \\
\hline 1 & & 10.803 & 5402.475 & 94439.453 & 0.5334 \\
\hline 2 & & 13.903 & 596081.375 & 17609554.000 & 99.4666 \\
\hline Total & & & 601483.850 & 17703993.453 & 100.0000 \\
\hline
\end{tabular}


(R)-4-((R)-2-(2-bromophenyl)-2-phenylethyl)-4-(4-methoxyphenyl)-3,4-dihydrobenzo[e][1,2,3]oxa thiazine 2,2-dioxide (4c)

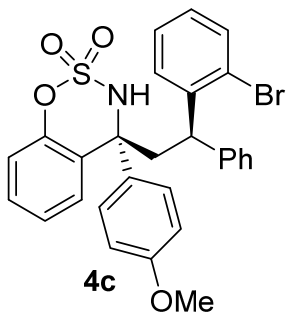

White solid, $34 \mathrm{mg}, 62 \%$ yield. $[\alpha]^{20}{ }_{\mathrm{D}}=-20.92$ (c 1.0, $\left.\mathrm{CHCl}_{3}\right) .{ }^{1} \mathrm{H}$ NMR $(300$ $\left.\mathrm{MHz}, \mathrm{CDCl}_{3}\right) \delta$ 7.59-7.28 (m, 13H), $7.22(\mathrm{~d}, J=8.2 \mathrm{~Hz}, 1 \mathrm{H}), 7.07-7.02(\mathrm{~m}, 1 \mathrm{H})$, $6.86(\mathrm{~d}, J=8.6 \mathrm{~Hz}, 2 \mathrm{H}), 4.78(\mathrm{~s}, 1 \mathrm{H}), 4.58(\mathrm{~d}, J=9.7 \mathrm{~Hz}, 1 \mathrm{H}), 3.79(\mathrm{~s}, 3 \mathrm{H})$, 3.29 (dd, $J=14.7,10.2 \mathrm{~Hz}, 1 \mathrm{H}), 2.89$ (d, $J=14.9 \mathrm{~Hz}, 1 \mathrm{H}) .{ }^{13} \mathrm{C}$ NMR (125 MHz, $\left.\mathrm{CDCl}_{3}\right) \delta 158.4,150.8,142.4,140.7,134.8,132.5,129.4,129.1,128.5,127.6$, $127.5,127.35,127.29,127.0,124.4,123.7,122.0,119.2,112.9,67.9,54.5,48.0$, 45.1. HRMS (ESI): $\mathrm{m} / \mathrm{z}$ [M-H] ${ }^{-}$calcd for $\mathrm{C}_{28} \mathrm{H}_{23} \mathrm{NO}_{4} \mathrm{SBr}$ : 548.0531; found: 548.0543.

The racemic stereoisomers of 1,2-adduct $\mathbf{4 c}$ were inseparable in HPLC. Hence, the optical purity of $\mathbf{4 c}$ was determined by its cyclized product $\mathbf{5 b}(99 \%$ ee). 
(R)-3-(2-phenyl-2-(p-tolyl)ethyl)benzo[d]isothiazole 1,1-dioxide (7)

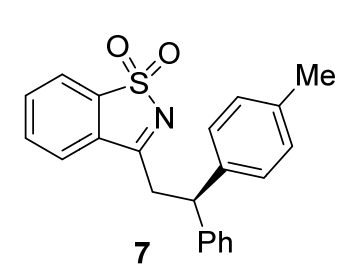

White solid, $17 \mathrm{mg}, 24 \%$ yield, $75 \%$ ee. $[\alpha]^{20}=-2.42\left(\mathrm{c} 1.0, \mathrm{CHCl}_{3}\right) .{ }^{1} \mathrm{H}$ NMR (300 MHz, $\left.\mathrm{CDCl}_{3}\right) \delta 7.86(\mathrm{~d}, J=6.9 \mathrm{~Hz}, 1 \mathrm{H}), 7.71-7.61(\mathrm{~m}, 2 \mathrm{H}), 7.54$ $(\mathrm{d}, J=7.7 \mathrm{~Hz}, 1 \mathrm{H}), 7.27(\mathrm{~s}, 4 \mathrm{H}), 7.17(\mathrm{~d}, J=6.6 \mathrm{~Hz}, 3 \mathrm{H}), 7.09$ (d, $J=7.7 \mathrm{~Hz}$, $2 \mathrm{H}), 4.80(\mathrm{t}, J=7.6 \mathrm{~Hz}, 1 \mathrm{H}), 3.67$ (d, $J=7.5 \mathrm{~Hz}, 2 \mathrm{H}), 2.28(\mathrm{~s}, 3 \mathrm{H}) .{ }^{13} \mathrm{C} \mathrm{NMR}$ $\left(125 \mathrm{MHz}, \mathrm{CDCl}_{3}\right) \delta 176.2,144.4,141.2,141.1,137.9,135.0,134.7,132.6$, $130.8,130.1,129.1,128.9,128.2,125.2,123.8,48.7,38.6,22.3$. HRMS (ESI): $\mathrm{m} / \mathrm{z}[\mathrm{M}-\mathrm{H}]^{-}$calcd for $\mathrm{C}_{22} \mathrm{H}_{18} \mathrm{NO}_{2} \mathrm{~S}: 360.1058$; found: 360.1057 .

HPLC: Chiralpak AS-H column $(250 \mathrm{~mm})$; detected at $224 \mathrm{~nm}$; hexane $/$ - -propanol = 70/30; flow = 0.6 $\mathrm{mL} / \mathrm{min}$; Retention time: $44.0 \mathrm{~min}, 52.9 \mathrm{~min}$ (major). 75\% ee.

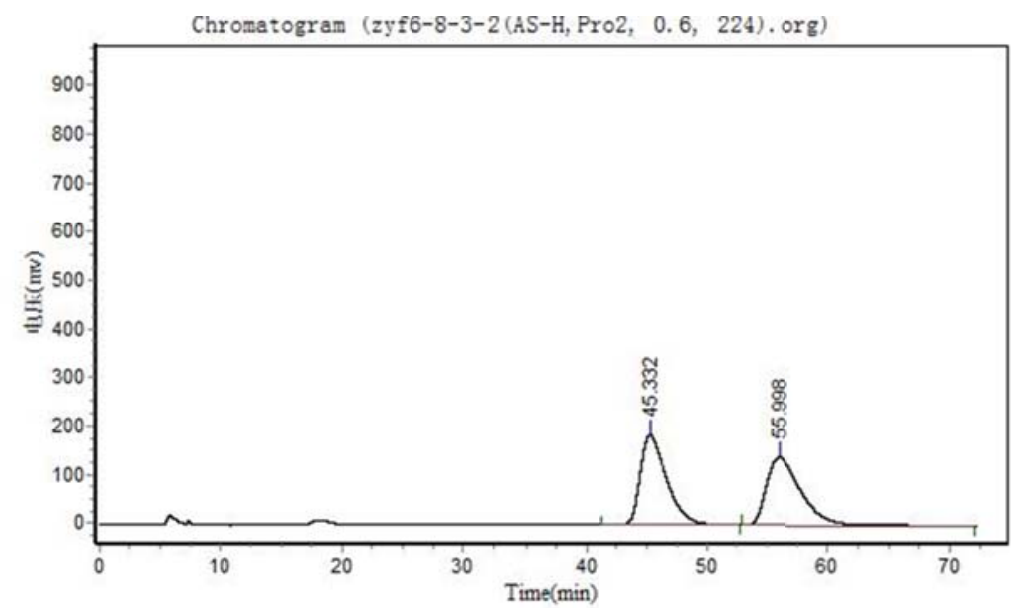

Results

\begin{tabular}{cccccc} 
Peak No. & Peak ID & Ret Time & Height & Area & Conc. \\
\hline 1 & 45.332 & 184526.578 & 28474694.000 & 49.9199 \\
2 & 55.998 & 139933.641 & 28566064.000 & 50.0801 \\
\hline Total & & 324460.219 & 57040758.000 & 100.0000
\end{tabular}

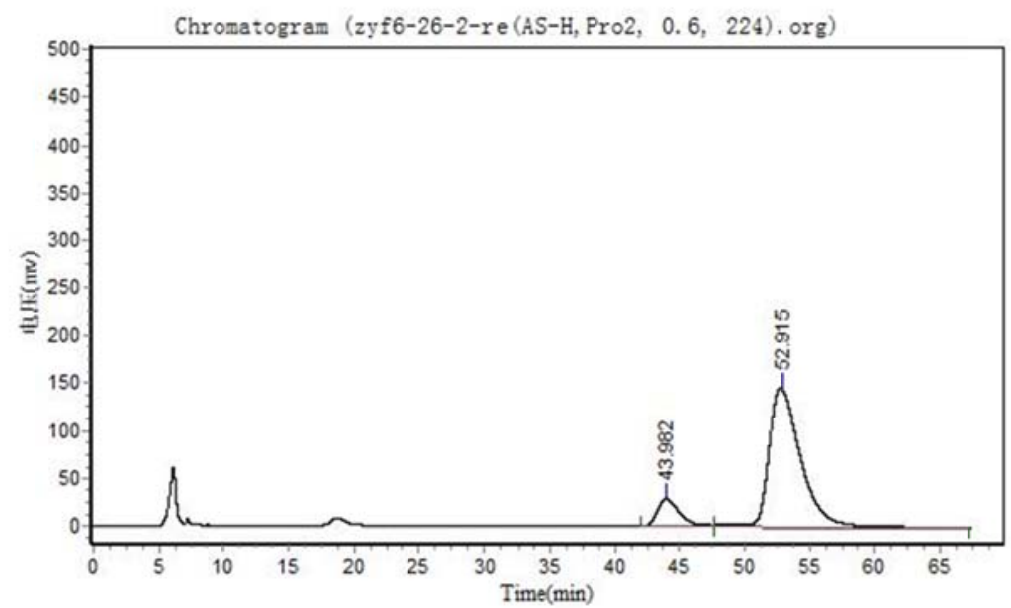

Results

\begin{tabular}{|c|c|c|c|c|c|}
\hline Peak No. & Peak ID & Ret Time & Height & Area & Conc. \\
\hline 1 & & 43.982 & 28690.211 & 3532331.250 & 12.3615 \\
\hline 2 & & 52.915 & 146064.688 & 25042842.000 & 87.6385 \\
\hline Total & & & 174754.898 & 28575173.250 & 100.0000 \\
\hline
\end{tabular}


(R)-3-((R)-2-phenyl-2-(p-tolyl)ethyl)-3-(p-tolyl)-2,3-dihydrobenzo[d]isothiazole 1,1-dioxide (8a)

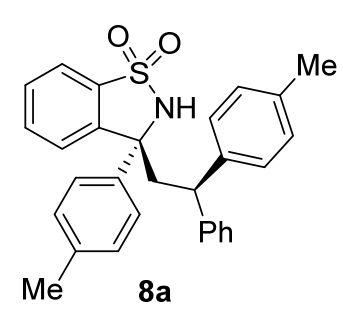

White solid, $85 \mathrm{mg}, 94 \%$ yield, $95 \%$ de. $[\alpha]^{20}{ }_{\mathrm{D}}=-49.12\left(\mathrm{c} 1.0, \mathrm{CHCl}_{3}\right) .{ }^{1} \mathrm{H}$ NMR (300 MHz, $\left.\mathrm{CDCl}_{3}\right) \delta$ 7.80-7.66 (m, 1H), 7.57-7.37 (m, 4H), 7.33-7.15 (m, 8H), 7.07-6.96 (m, 4H), $4.52(\mathrm{~s}, 1 \mathrm{H}), 4.08(\mathrm{dd}, J=9.6,3.5 \mathrm{~Hz}, 1 \mathrm{H})$, 3.25-3.09 (m, 2H), 2.36 (s, 3H), 2.29 (s, 3H). ${ }^{13} \mathrm{C}$ NMR $\left(125 \mathrm{MHz}, \mathrm{CDCl}_{3}\right) \delta$ 144.3, 142.8, 139.3, 138.1, 137.2, 136.3, 133.1, 132.4, 129.3, 129.1, 128.5, 128.2, 127.3, 126.6, 125.9, 125.3, 123.8, 120.6, 68.0, 46.3, 45.5, 20.4, 20.3. HRMS (ESI): $m / z$ [M-H] ${ }^{-}$calcd for $\mathrm{C}_{29} \mathrm{H}_{26} \mathrm{NO}_{2} \mathrm{~S}: 452.1684$; found: 452.1681 .

HPLC: Chiralpak AD-H column $(250 \mathrm{~mm})$; detected at $224 \mathrm{~nm}$; hexane/i-propanol = 70/30; flow = 0.7 $\mathrm{mL} / \mathrm{min}$; Retention time: $13.0 \mathrm{~min}, 16.1 \mathrm{~min}$ (major), $29.7 \mathrm{~min}, 32.2 \mathrm{~min}$. 95\% de for $\mathbf{8 a}$.

The reaction was carried out with ketimine $\mathbf{6}$ and 2.0 equiv of arylboronic acid 2a in the presence of 5.0 $\mathrm{mol} \%$ of $[\mathrm{Rh}(\mathrm{COD}) \mathrm{Cl}], \mathrm{K}_{2} \mathrm{HPO}_{4}(1.5 \mathrm{M}, 0.5$ equiv) in $1.0 \mathrm{~mL}$ toluene at $\mathrm{rt}$ for $12 \mathrm{~h}$ to afford 8 with four stereoisomers. The corresponding HPLC spectrum of $\mathbf{8}$ is shown in Figure 3a.

The reaction of $(R)-7$ (75\% ee) with $p$-tolylboronic acid 2 a was conducted under rhodium catalysis by using $\mathbf{L} 7(<10 \%$ ee) as a ligand to afford $\mathbf{8}$. The corresponding HPLC spectrum of $\mathbf{8}$ is shown in Figure $3 \mathbf{b}$.

The reaction of ketimine 6 and $p$-tolylboronic acid 2a (3 equiv) was performed under rhodium catalysis with optically pure $\mathbf{L} 7$ as a ligand for $24 \mathrm{~h}$. The corresponding HPLC spectrum of $\mathbf{8}$ is shown in Figure 3c.

The reaction of ketimine $\mathbf{6}$ and $p$-tolylboronic acid $\mathbf{2 a}$ (2 equiv) was performed under rhodium catalysis with optically pure $\mathbf{L} 7$ as a ligand for 12 h. The corresponding HPLC spectrum of $\mathbf{8}$ is shown in Figure 3d.

The reaction of $(R)-7$ (75\% ee) and p-tolylboronic acid $\mathbf{2 a}$ (2 equiv) was performed under rhodium catalysis with optically pure $\mathbf{L} 7$ as a ligand for $12 \mathrm{~h}$. The corresponding HPLC spectrum of $\mathbf{8}$ is shown in Figure 3e. 


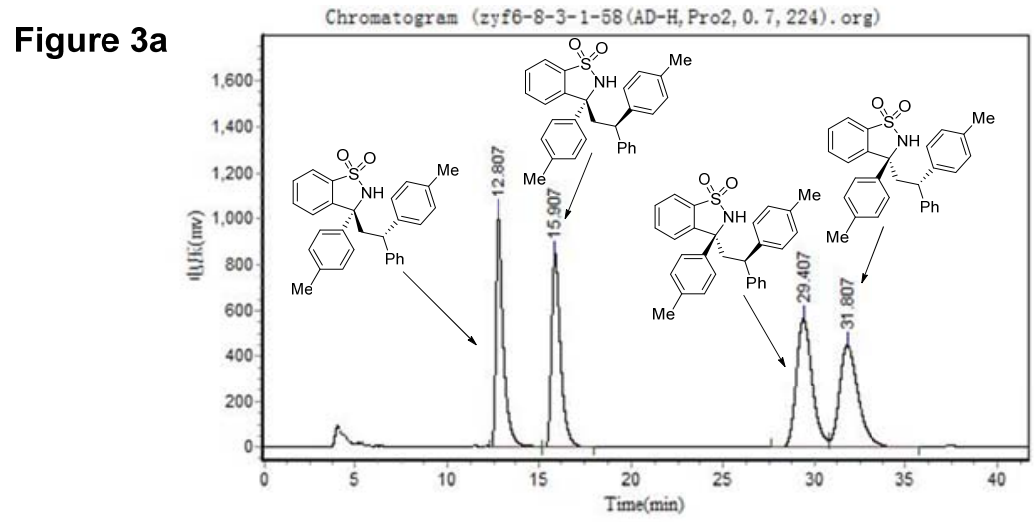

\begin{tabular}{|c|c|c|c|c|c|}
\hline \multicolumn{6}{|c|}{ Results } \\
\hline Peak No. & Peak ID & Ret Time & Height & Area & Conc. \\
\hline 1 & & 12.807 & 1027435.688 & 31298430.000 & 24.7519 \\
\hline 2 & & 15.907 & $\$ 46725.063$ & 28857786.000 & 22.8217 \\
\hline 3 & & 29.407 & 565003.063 & 33988048.000 & 26.8789 \\
\hline 4 & & 31.807 & 451574.656 & 32304400.000 & 25.5474 \\
\hline Total & & & 2890738.469 & 126448664.000 & 100.0000 \\
\hline
\end{tabular}
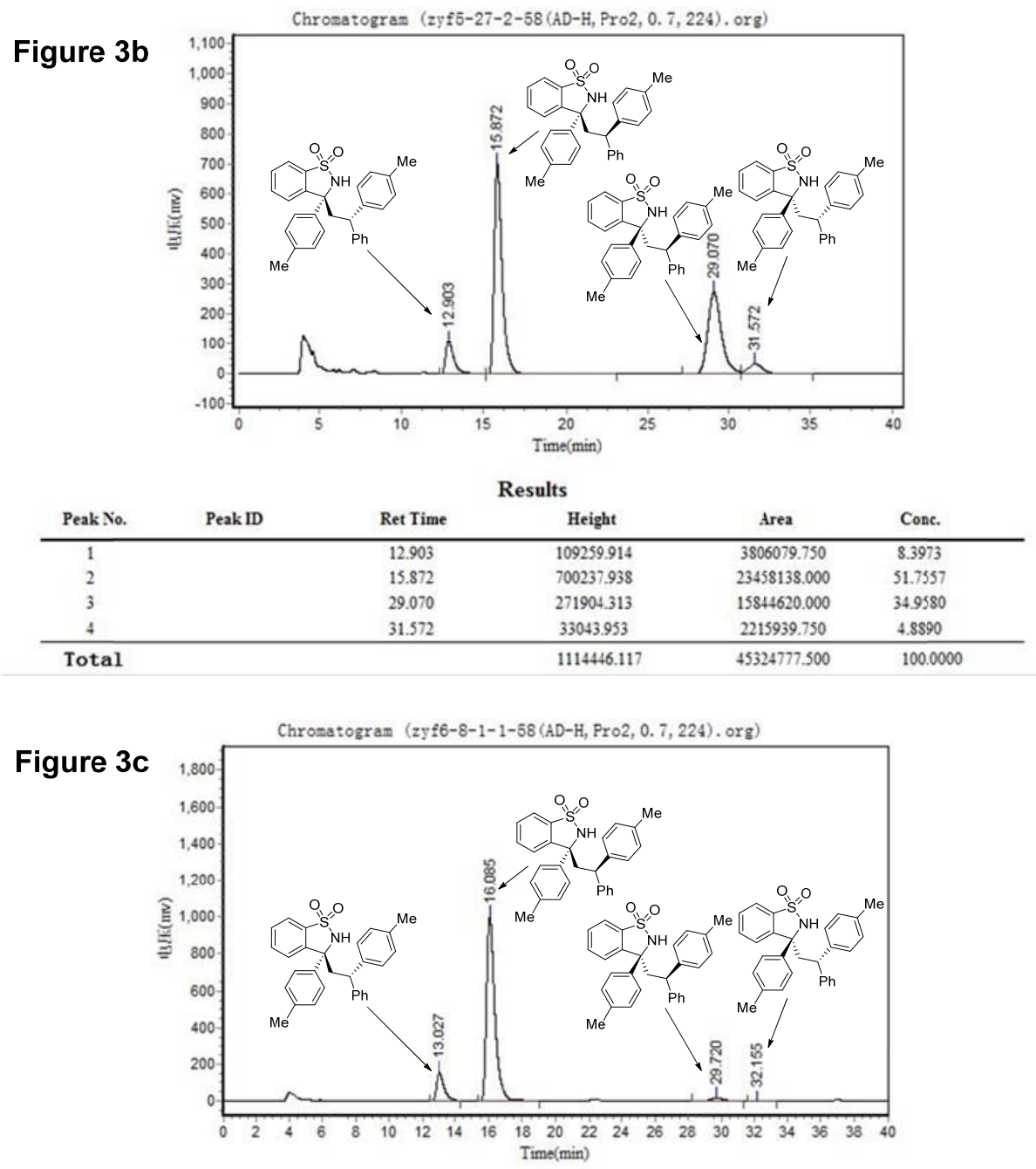

\begin{tabular}{|c|c|c|c|c|c|}
\hline \multicolumn{6}{|c|}{ Results } \\
\hline Peak No. & Peak ID & Ret Time & Height & Area & Conc. \\
\hline 1 & & 13.027 & 158971.391 & 5295488.000 & 12.7854 \\
\hline 2 & & 16.085 & 1002982.563 & 35081696.000 & 84,7010 \\
\hline 3 & & 29.720 & 17706.895 & 962004.500 & 2.3227 \\
\hline 4 & & 32.155 & 1711.141 & 79086.102 & 0.1909 \\
\hline Total & & & 1181371.989 & 41418274.602 & 100.0000 \\
\hline
\end{tabular}




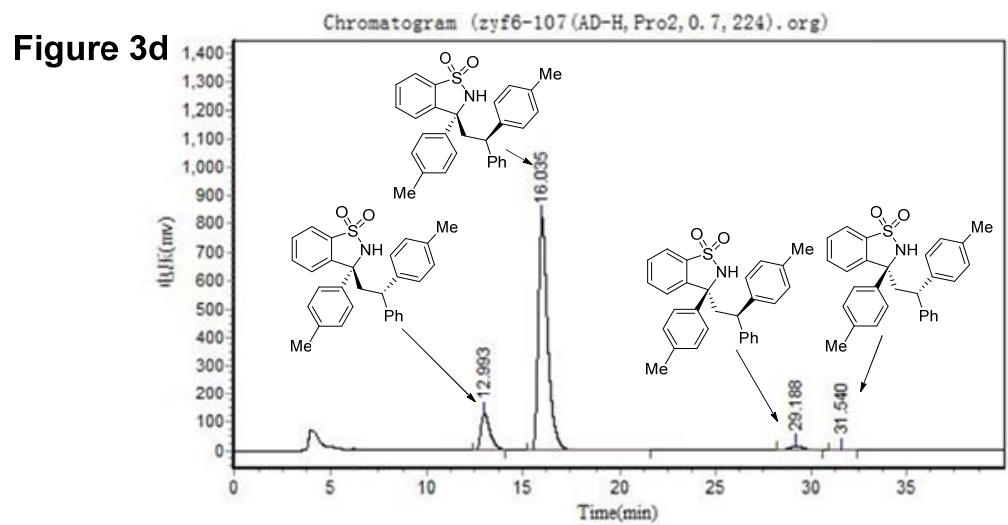

\begin{tabular}{|c|c|c|c|c|c|}
\hline \multicolumn{6}{|c|}{ Results } \\
\hline Peak No. & Peak ID & Ret Time & Height & Area & Conc. \\
\hline 1 & & 12.993 & 130090.031 & 4267338.500 & 12.9356 \\
\hline 2 & & 16.035 & $\$ 16362.875$ & 27896474.000 & 84.5629 \\
\hline 3 & & 29.188 & 14582.870 & 760051.938 & 2.3040 \\
\hline 4 & & 31.540 & 1431.541 & 65167.004 & 0.1975 \\
\hline Total & & & 962467.317 & 32989031.441 & 100.0000 \\
\hline
\end{tabular}

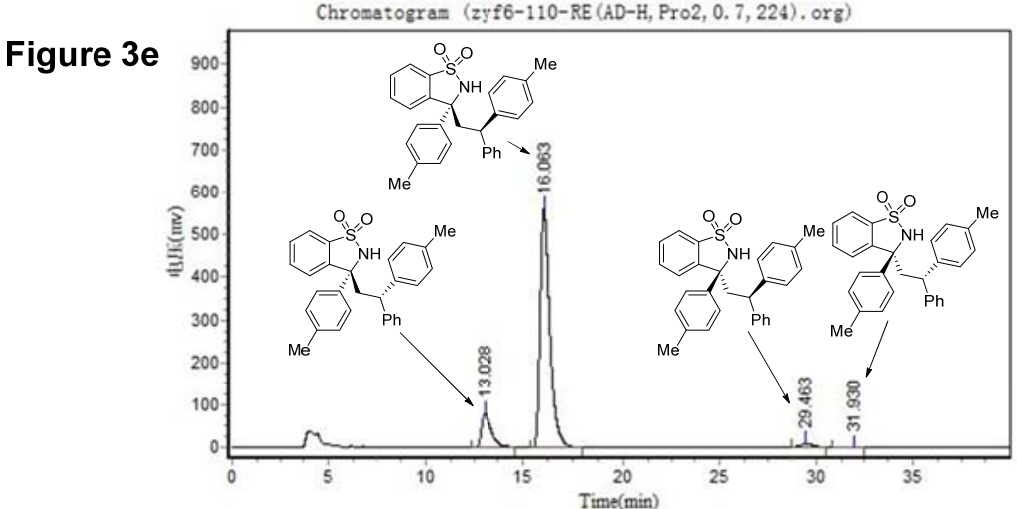

\begin{tabular}{|c|c|c|c|c|c|}
\hline \multicolumn{6}{|c|}{ Results } \\
\hline Peak No. & Peak ID & Ret Time & Height & Area & Conc. \\
\hline 1 & & 13.028 & 80081.680 & 2838056.750 & 12.5330 \\
\hline 2 & & 16.063 & 560585.250 & 19325396.000 & 85.3417 \\
\hline 3 & & 29.463 & 9141.274 & 444944.438 & 1.9649 \\
\hline 4 & & 31.930 & 915.584 & 36329.797 & 0.1604 \\
\hline Total & & & 650723.789 & 22644726.984 & 100.000 \\
\hline
\end{tabular}


6. Synthetic Transformations of Arylation Products

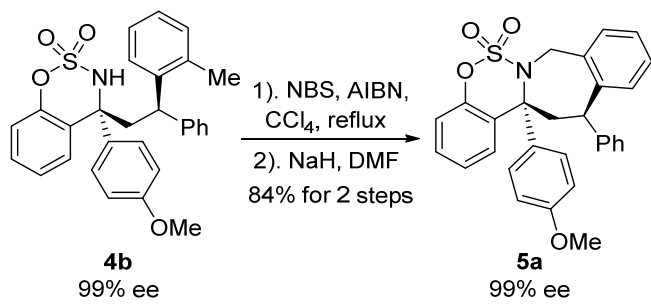

Procedure: under argon atmosphere, a round bottom flask was charged with compound $\mathbf{4 b}(0.2 \mathrm{mmol}$, 1.0 equiv), NBS ( $0.2 \mathrm{mmol}, 1.0$ equiv), AIBN ( $0.02 \mathrm{mmol}, 0.1$ equiv) and $5 \mathrm{~mL}$ of $\mathrm{CCl}_{4}$. The resulting mixture was stirred at refluxing temperature overnight. After cooling to room termperature, saturated aq $\mathrm{Na}_{2} \mathrm{CO}_{3}$ solution was added to quench the reaction and the mixture was then extracted with ethyl acetate $(15 \mathrm{~mL} \times 3)$. The combined organic layers were washed with brine, dried over anhydrous $\mathrm{Na}_{2} \mathrm{SO}_{4}$ and evaporated to dryness to afford the crude bromination product, which was used without further purification in the next cyclization step.

The above obtained crude product in DMF $(1 \mathrm{~mL})$ solution was added to a glass reaction vessel charged with $\mathrm{NaH}\left(0.22 \mathrm{mmol}, 1.1\right.$ equiv) and fresh distilled DMF $(1 \mathrm{~mL})$ at $0{ }^{\circ} \mathrm{C}$ under argon atmosphere. The resulting solution was then stirred at ambient temperature for $3 \mathrm{~h}$. After being quenched with water, the mixture was extracted with ethyl acetate $(15 \mathrm{~mL} \times 3)$. The combined organic phase was then washed subsequently with $1 \mathrm{~N} \mathrm{HCl}$, brine, dried over $\mathrm{Na}_{2} \mathrm{SO}_{4}$ and concentrated. The residue was purified by silica gel flash chromatography using petroleum ether/ethyl acetate as an eluent to afford compound $\mathbf{5 a}$ in $84 \%$ yield for 2 steps.

$(13 R, 14 a R)-14 a-(4-m e t h o x y p h e n y l)-13-p h e n y l-8,13,14,14 a-t e t r a h y d r o b e n z o[e] b e n z o[5,6][1,2,3]$ ox athiazino[3,4-a]azepine 6,6-dioxide (5a)

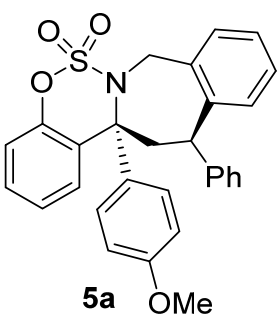

White solid, $81 \mathrm{mg}, 84 \%$ yield, $99 \%$ ee. $[\alpha]^{20}{ }_{\mathrm{D}}=-239.20\left(\mathrm{c} 1.0, \mathrm{CHCl}_{3}\right) .{ }^{1} \mathrm{H}$ NMR $\left(300 \mathrm{MHz}, \mathrm{CDCl}_{3}\right) \delta$ 7.67-7.32 (m, 8H), 7.16-7.08 (m, 7H), $6.86(\mathrm{~d}, J=$ $8.1 \mathrm{~Hz}, 1 \mathrm{H}), 6.50(\mathrm{~d}, J=7.6 \mathrm{~Hz}, 1 \mathrm{H}), 4.67(\mathrm{dd}, J=13.2,7.7 \mathrm{~Hz}, 2 \mathrm{H}), 4.46(\mathrm{~d}, J$ $=15.8 \mathrm{~Hz}, 1 \mathrm{H}), 3.82(\mathrm{~s}, 3 \mathrm{H}), 3.41(\mathrm{dd}, J=14.7,10.9 \mathrm{~Hz}, 1 \mathrm{H}), 3.12(\mathrm{~d}, J=14.7$ $\mathrm{Hz}, 1 \mathrm{H}) .{ }^{13} \mathrm{C}$ NMR $\left(125 \mathrm{MHz}, \mathrm{CDCl}_{3}\right) \delta 158.6,146.6,143.3,142.7,134.4,133.0$, 129.2, 128.4, 128.13, 128.07, 127.8, 126.7, 126.5, 125.9, 125.1, 118.4, 114.1, 72.2, 54.7, 48.7, 44.3, 40.1. HRMS (EI): $\mathrm{m} / \mathrm{z}$ [M] ${ }^{+}$calcd for $\mathrm{C}_{29} \mathrm{H}_{25} \mathrm{NO}_{4} \mathrm{~S}: 483.1504$; found: 483.1503 .

HPLC: Chiralpak IC column $(250 \mathrm{~mm})$; detected at $224 \mathrm{~nm}$; hexane $/ \mathrm{i}$-propanol $=95 / 5$; flow $=0.7$ $\mathrm{mL} / \mathrm{min}$; Retention time: $47.0 \mathrm{~min}, 51.4 \mathrm{~min}$ (major). 99\% ee.

The reaction of $( \pm)$-3d with $p$-methoxyphenylboronic acid $\mathbf{2 b}$ was conducted under rhodium catalysis by using $\mathbf{L} 7(<10 \%$ ee) as a ligand. The expected 1,2-addition could take place with concomitant formation of four stereoisomers of $\mathbf{4 b}$, which was then converted to $\mathbf{5 a}$ with four stereoisomers through the steps mentioned above. The corresponding HPLC spectrum of 5a is shown in Figure 4a .

The reaction of $(R)$-3d ( $99 \%$ ee) with $p$-methoxyphenylboronic acid $\mathbf{2 b}$ was conducted under rhodium catalysis by using optically pure $\mathbf{L} \mathbf{1}$ as a ligand to afford $\mathbf{4 b}$. From 1,2-adduct $\mathbf{4 b}$ with expected high diastereomeric excess, $\mathbf{5 a}$ could be obtained as a sole stereoisomer through the steps mentioned above. The corresponding HPLC spectrum of $\mathbf{5 a}$ is shown in Figure $\mathbf{4 b}$. 


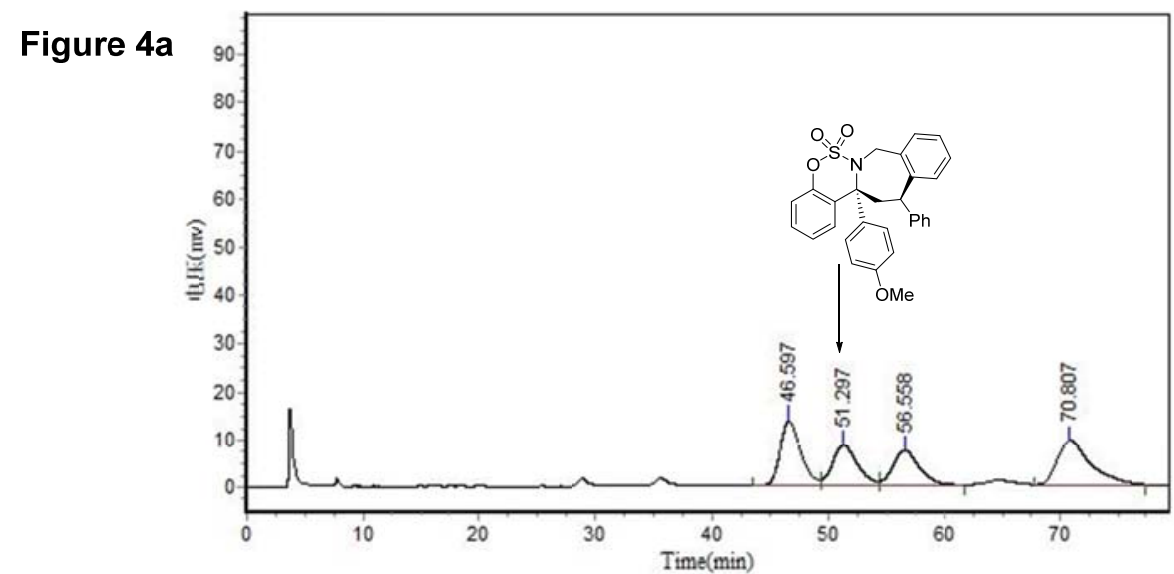

\begin{tabular}{|c|c|c|c|c|c|}
\hline \multicolumn{6}{|c|}{ Results } \\
\hline Peak No. & Peak ID & Ret Time & Height & Area & Conc. \\
\hline 1 & & 46.597 & 13805.668 & 1739776.625 & 28.2811 \\
\hline 2 & & 51.297 & 8609.648 & 1303417.250 & 21.1879 \\
\hline 3 & & 56.558 & 7446.830 & 1251253.000 & 20.3399 \\
\hline 4 & & 70.807 & 9359.118 & 1857272.875 & 30.1911 \\
\hline Total & & & 39221.264 & 6151719.750 & 100.0000 \\
\hline
\end{tabular}

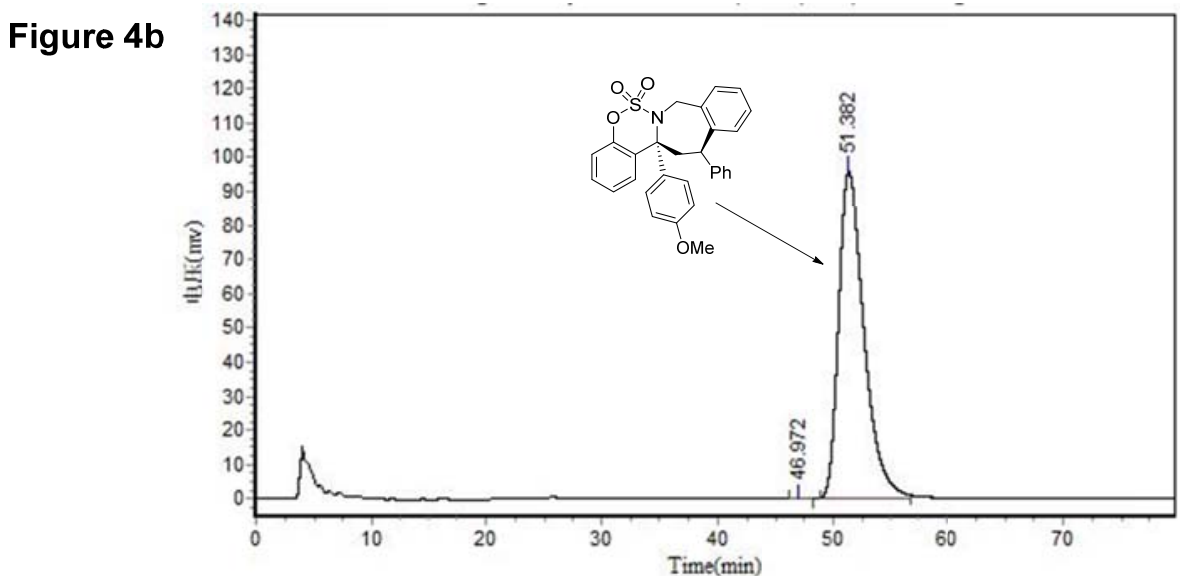

\begin{tabular}{|c|c|c|c|c|c|}
\hline \multicolumn{6}{|c|}{ Results } \\
\hline Peak No. & Peak ID & Ret Time & Height & Area & Conc. \\
\hline 1 & & 46.972 & 212.776 & 15987.799 & 0.1101 \\
\hline 2 & & 51.382 & 95661.234 & 14502510.000 & 99.8899 \\
\hline Total & & & 95874.010 & 14518497.799 & 100.0000 \\
\hline
\end{tabular}



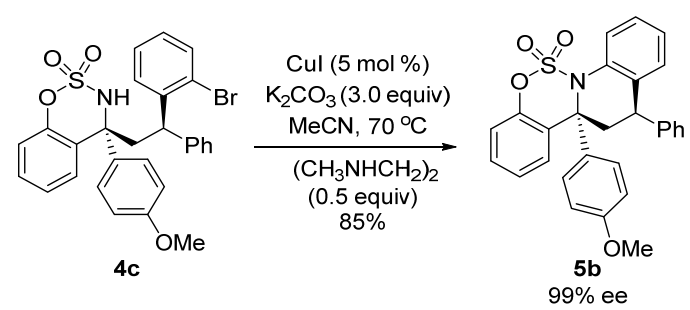

Procedure: under argon atmosphere, a solution of $\mathbf{4 c}(0.2 \mathrm{mmol}, 1.0$ equiv), CuI (5 mol \%), $N, N^{\prime}$-dimethylethanediamine ( 0.5 equiv) and $\mathrm{K}_{2} \mathrm{CO}_{3}$ (3 equiv) in $1.0 \mathrm{~mL}$ of $\mathrm{MeCN}$ was stirred at $70{ }^{\circ} \mathrm{C}$ overnight. After EtOAc $(10 \mathrm{~mL})$ was added, the mixture was then passed through a pad of silica gel. The filtrate was concentrated under reduced pressure and the residue was purified by silica gel column chromatography using petroleum ether/ethyl acetate as an eluent to afford the product $\mathbf{5 b}$ in $85 \%$ yield.

(11bR,13R)-11b-(4-methoxyphenyl)-13-phenyl-11b,12-dihydro-13H-benzo[5,6][1,2,3] oxathiazino[ 3,4-a]quinoline 6,6-dioxide (5b)<smiles>COc1ccc([C@H]2CC(c3ccccc3)c3ccccc3N2S(=O)(=O)O)cc1</smiles>
White solid, $80 \mathrm{mg}, 85 \%$ yield, $99 \%$ ee. $[\alpha]^{20}{ }_{\mathrm{D}}=-297.17\left(\mathrm{c} 1.0, \mathrm{CHCl}_{3}\right) .{ }^{1} \mathrm{H} \mathrm{NMR}$ $\left(300 \mathrm{MHz}, \mathrm{CDCl}_{3}\right) \delta$ 7.68-7.54 (m, 3H), $7.31(\mathrm{~d}, J=4.2 \mathrm{~Hz}, 4 \mathrm{H}), 7.25-7.03(\mathrm{~m}$, $6 \mathrm{H}), 6.99-6.94(\mathrm{~m}, 1 \mathrm{H}), 6.80(\mathrm{dd}, J=13.2,8.3 \mathrm{~Hz}, 3 \mathrm{H}), 4.26(\mathrm{dd}, J=11.3,7.8 \mathrm{~Hz}$, $1 \mathrm{H}), 3.72(\mathrm{~s}, 3 \mathrm{H}), 3.37(\mathrm{dd}, J=14.6,11.7 \mathrm{~Hz}, 1 \mathrm{H}), 3.10(\mathrm{dd}, J=14.8,7.7 \mathrm{~Hz}, 1 \mathrm{H})$. ${ }^{13} \mathrm{C}$ NMR $\left(125 \mathrm{MHz}, \mathrm{CDCl}_{3}\right) \delta 159.4,147.4,144.9,134.0,133.9,133.2,130.6$, $130.1,129.5,129.20,129.16,128.5,128.3,128.1,127.3,127.1,126.9,126.6$, 119.3, 114.6, 70.2, 55.5, 42.0, 39.6. HRMS (EI): $\mathrm{m} / \mathrm{z}[\mathrm{M}]^{+}$calcd for $\mathrm{C}_{28} \mathrm{H}_{23} \mathrm{NO}_{4} \mathrm{~S}$ :

469.1348; found: 469.1347 .

HPLC: Chiralpak AD-H column $(250 \mathrm{~mm})$; detected at $224 \mathrm{~nm}$; hexane $/ \mathrm{i}$-propanol $=80 / 20$; flow $=0.7$ $\mathrm{mL} / \mathrm{min}$; Retention time: $9.3 \mathrm{~min}, 18.9$ min (major). $99 \%$ ee.

The reaction of $( \pm)-\mathbf{3 e}$ with $p$-methoxyphenylboronic acid $\mathbf{2} \mathbf{b}$ was conducted under rhodium catalysis by using $\mathbf{L} 7(<10 \%$ ee) as a ligand. The expected 1,2-addition could take place with concomitant formation of four stereoisomers of $\mathbf{4 c}$, which was then converted to $\mathbf{5 b}$ with four stereoisomers through the steps mentioned above. The corresponding HPLC spectrum of $\mathbf{5 b}$ is shown in Figure $\mathbf{5 a}$.

The reaction of $(R)-\mathbf{3 e}(99 \%$ ee) with $p$-methoxyphenylboronic acid $\mathbf{2 b}$ was conducted under rhodium catalysis by using optically pure $\mathbf{L} 1$ as a ligand to afford $\mathbf{4 c}$. From 1,2-adduct $\mathbf{4 c}$ with expected high diastereomeric excess, $\mathbf{5 b}$ could be obtained as a sole stereoisomer through the steps mentioned above. The corresponding HPLC spectrum of $\mathbf{5 b}$ is shown in Figure $\mathbf{5 b}$. 


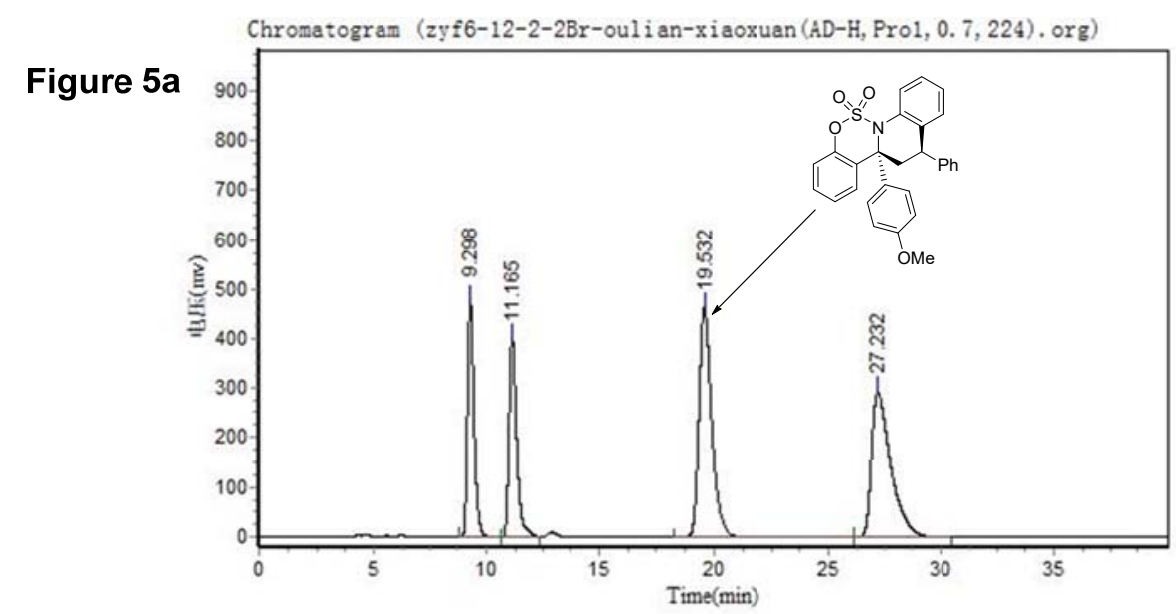

Results

\begin{tabular}{|c|c|c|c|c|c|}
\hline \multicolumn{6}{|c|}{ Results } \\
\hline Peak No. & Peak ID & Ret Time & Height & Area & Conc. \\
\hline 1 & & 9.298 & 477696.656 & 9572367.000 & 17.5267 \\
\hline 2 & & 11.165 & 404888.000 & 9532106.000 & 17.4530 \\
\hline 3 & & 19.532 & 463265.875 & 18159098.000 & 33.2487 \\
\hline 4 & & 27.232 & 293619.625 & 17352390.000 & 31.7716 \\
\hline Total & & & 1639470.156 & 54615961.000 & $100.000 \mathrm{C}$ \\
\hline
\end{tabular}

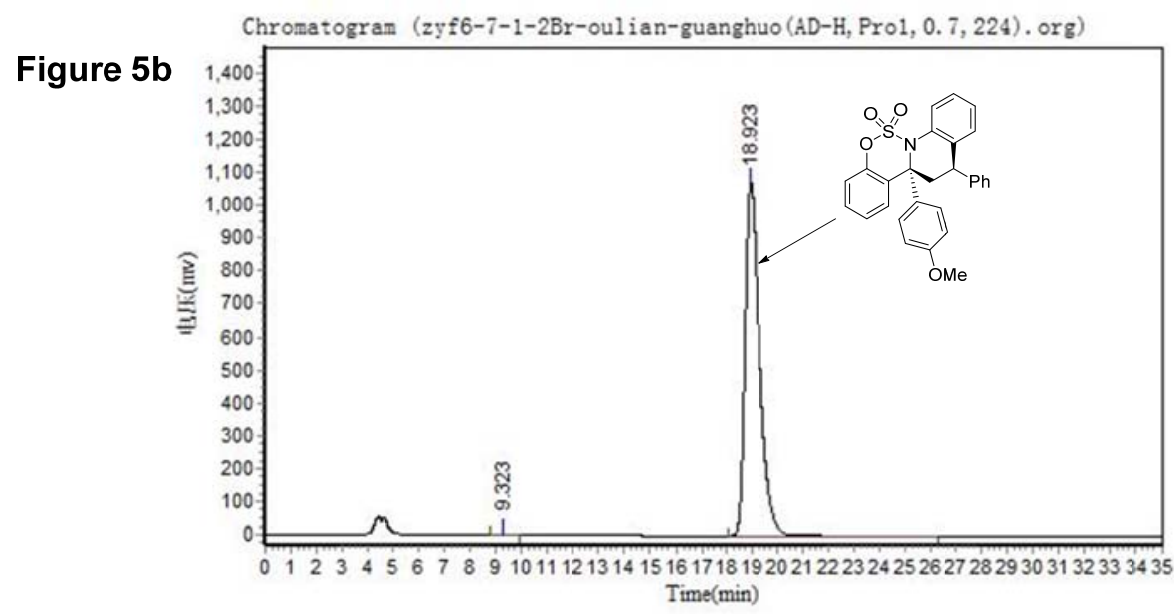

\begin{tabular}{|c|c|c|c|c|c|}
\hline \multicolumn{6}{|c|}{ Results } \\
\hline Peak No. & Peak ID & Ret Time & Height & Area & Conc. \\
\hline 1 & & 9.323 & 1402.424 & 35039.398 & 0.0824 \\
\hline 2 & & 18.923 & 1069437.250 & 42512788.000 & 99.9176 \\
\hline Total & & & 1070839.674 & 42547827.398 & $100.000 \mathrm{C}$ \\
\hline
\end{tabular}




\section{Copies of ${ }^{1} \mathrm{H}$ NMR and ${ }^{13} \mathrm{C}$ NMR spectra}

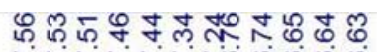

NNNNNNNG

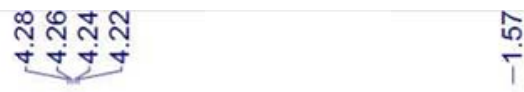

(1)

$\int^{\infty} \overline{\dot{m}}[\stackrel{0}{\stackrel{0}{-}}$
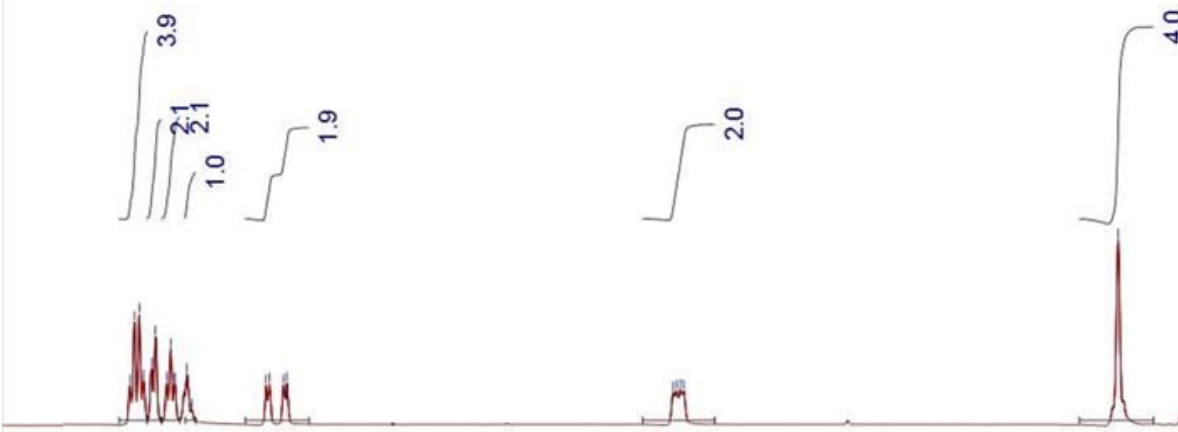

M
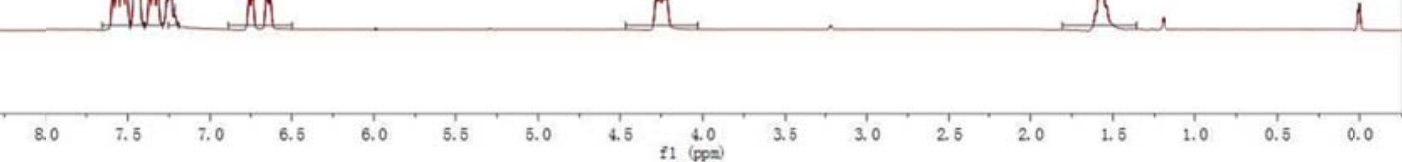

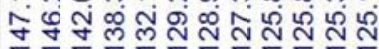

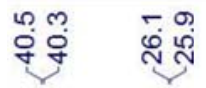
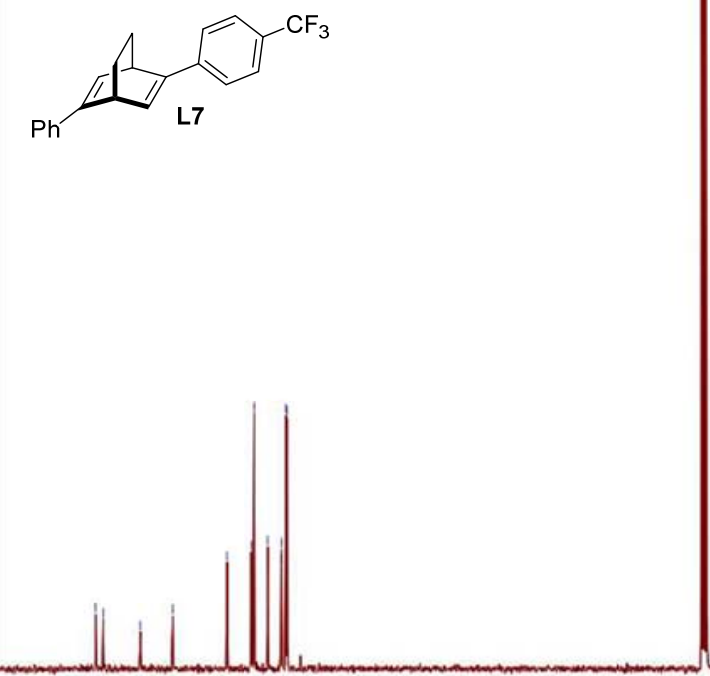

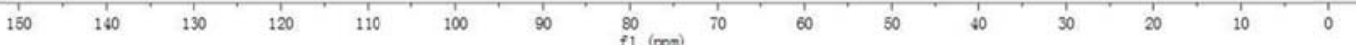



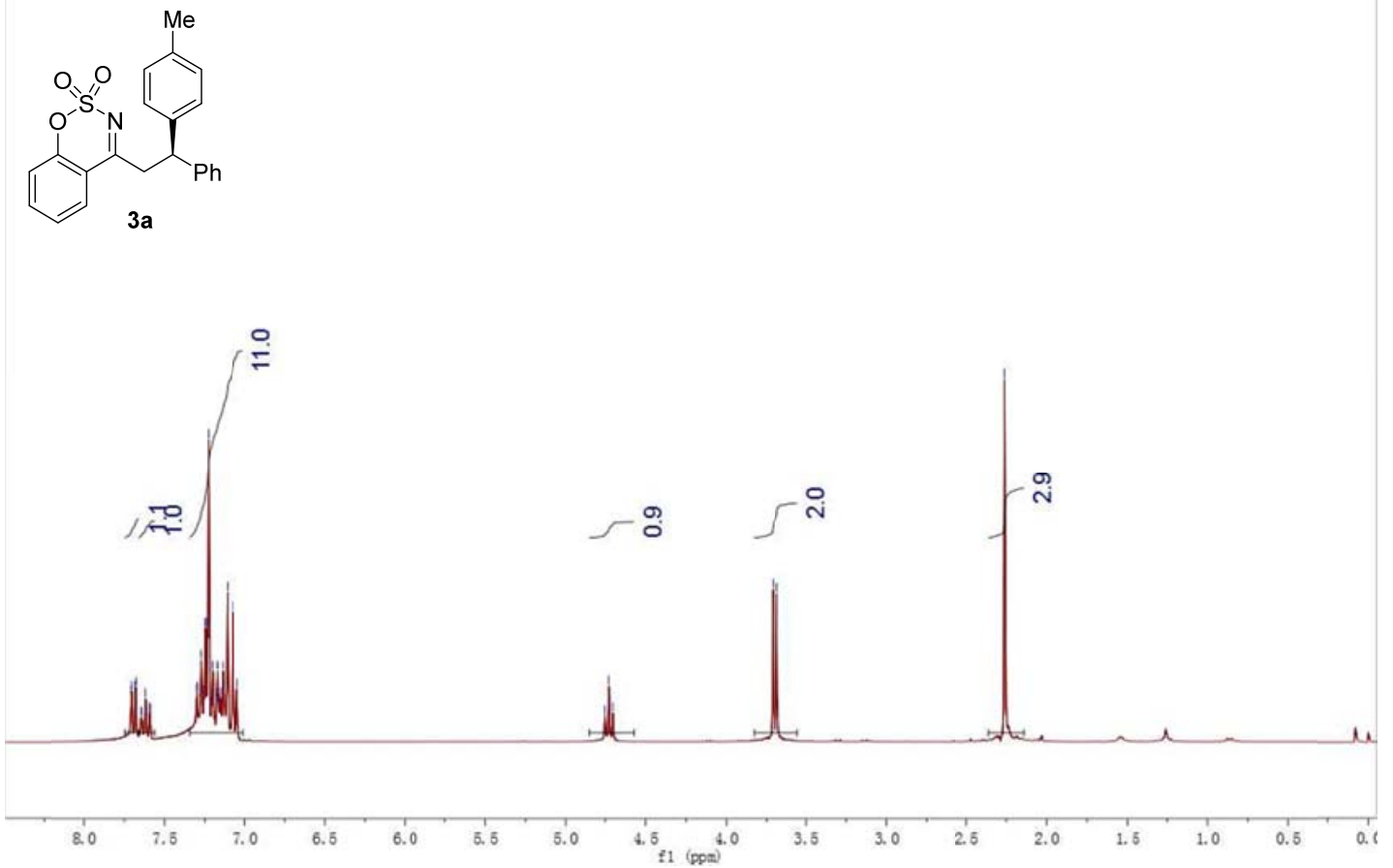

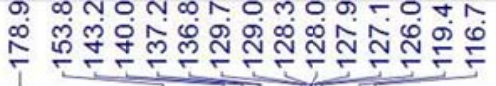

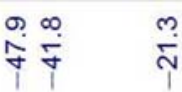

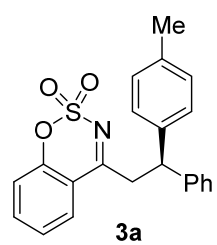

$3 a$

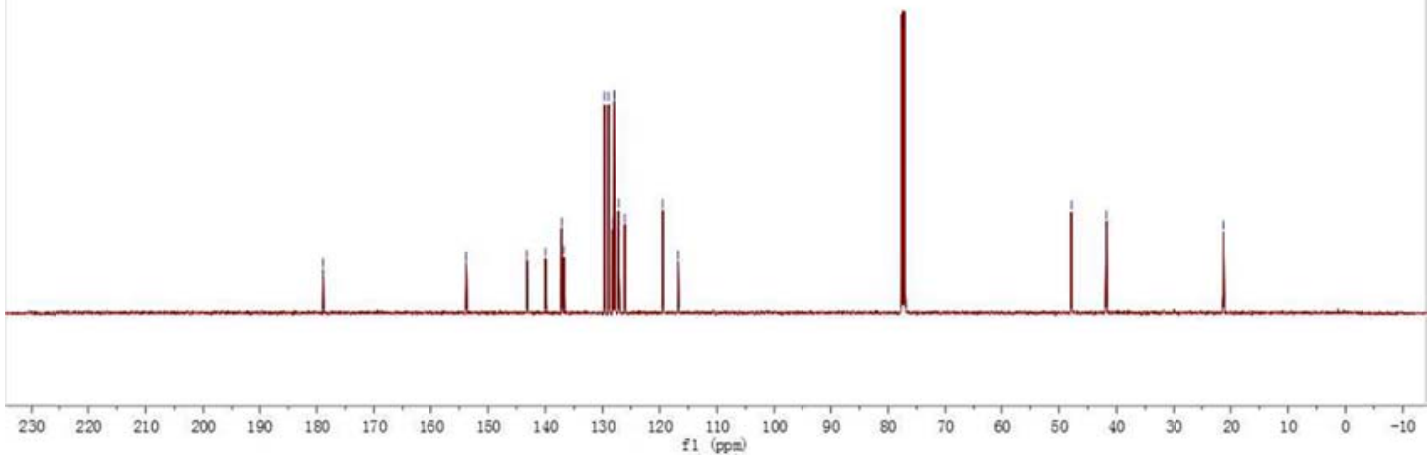




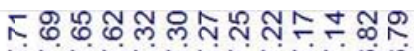

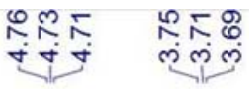
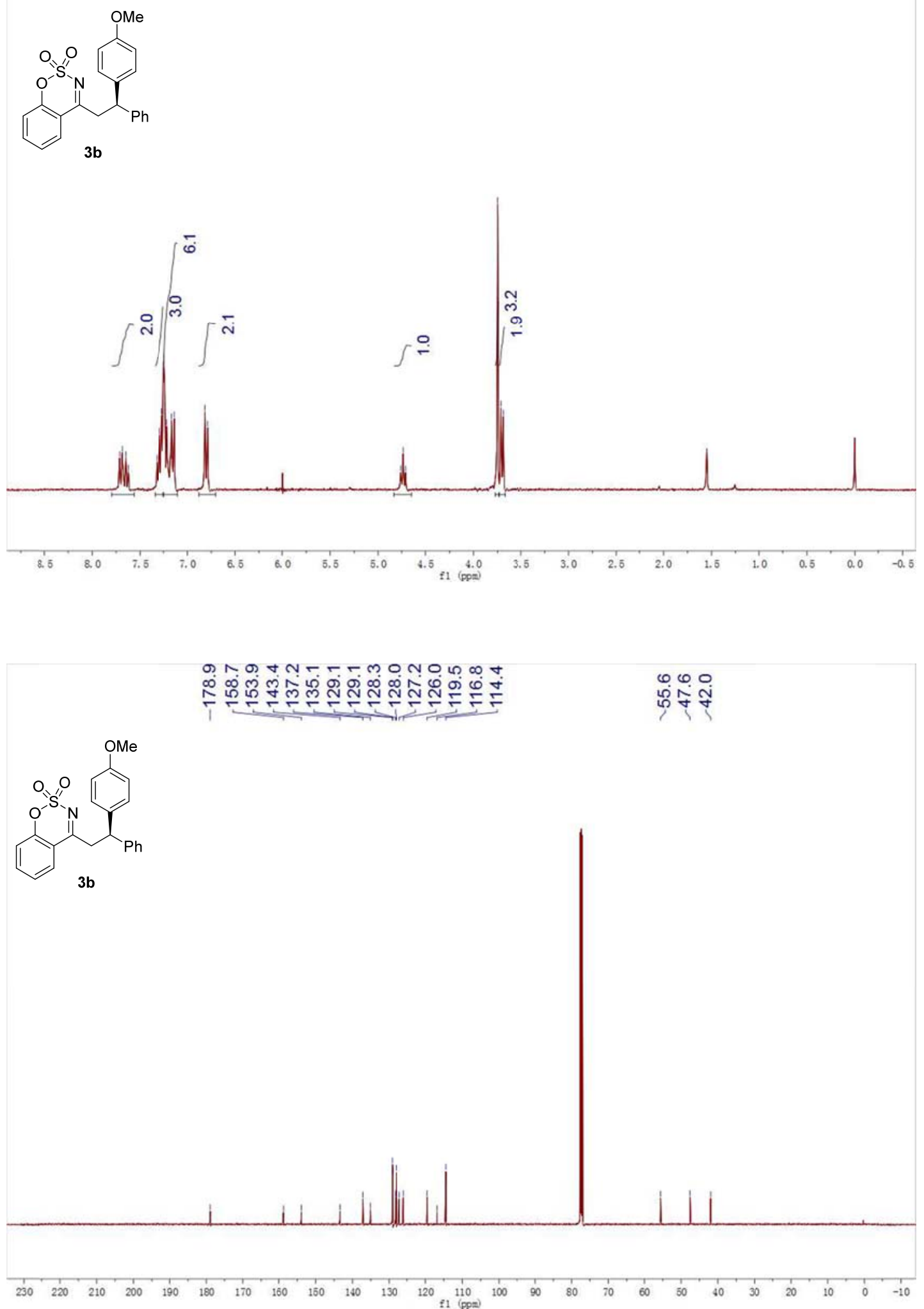


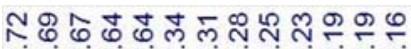

NNNNNNNNNNN
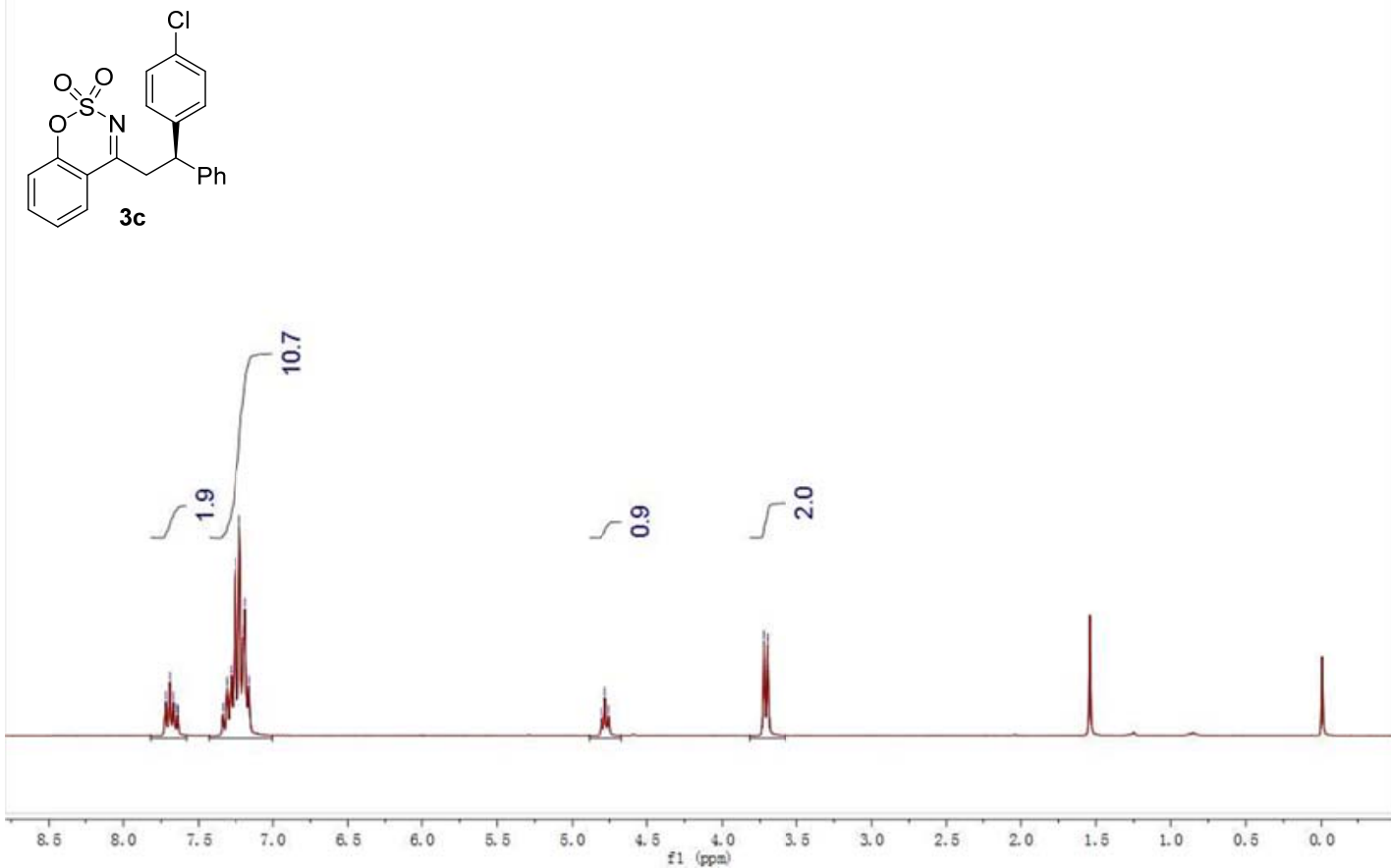

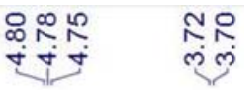

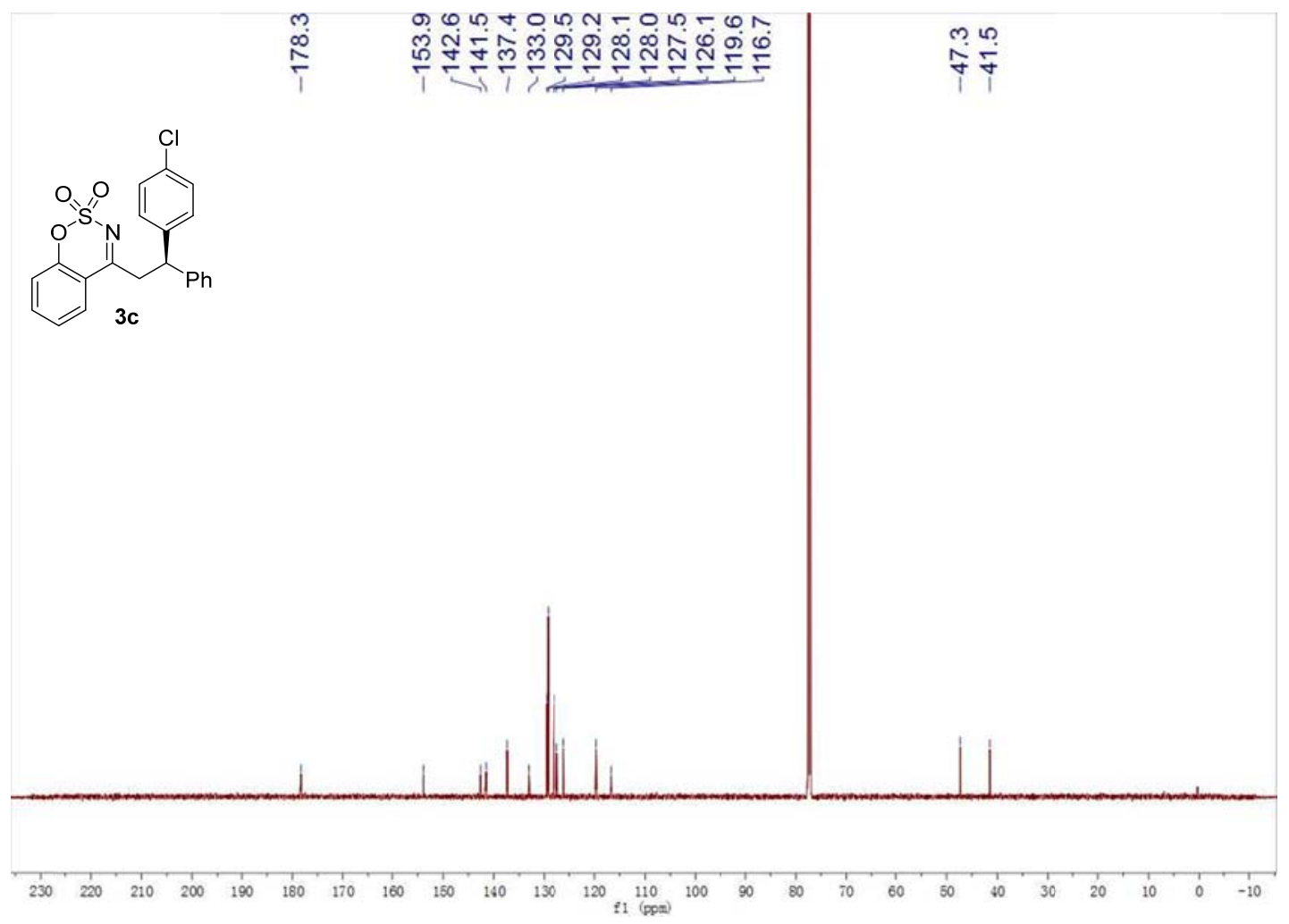




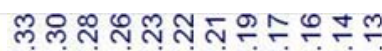

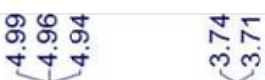

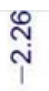

$\underbrace{M e}_{3 d}$

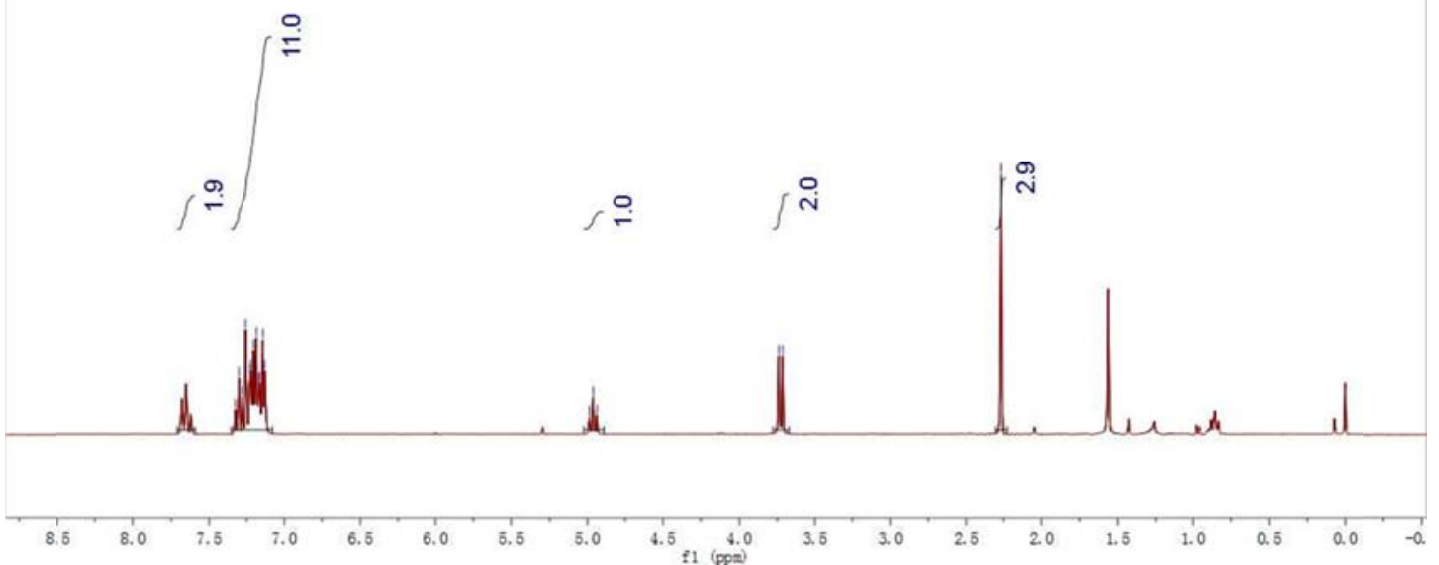

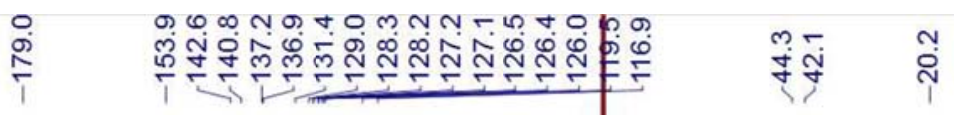
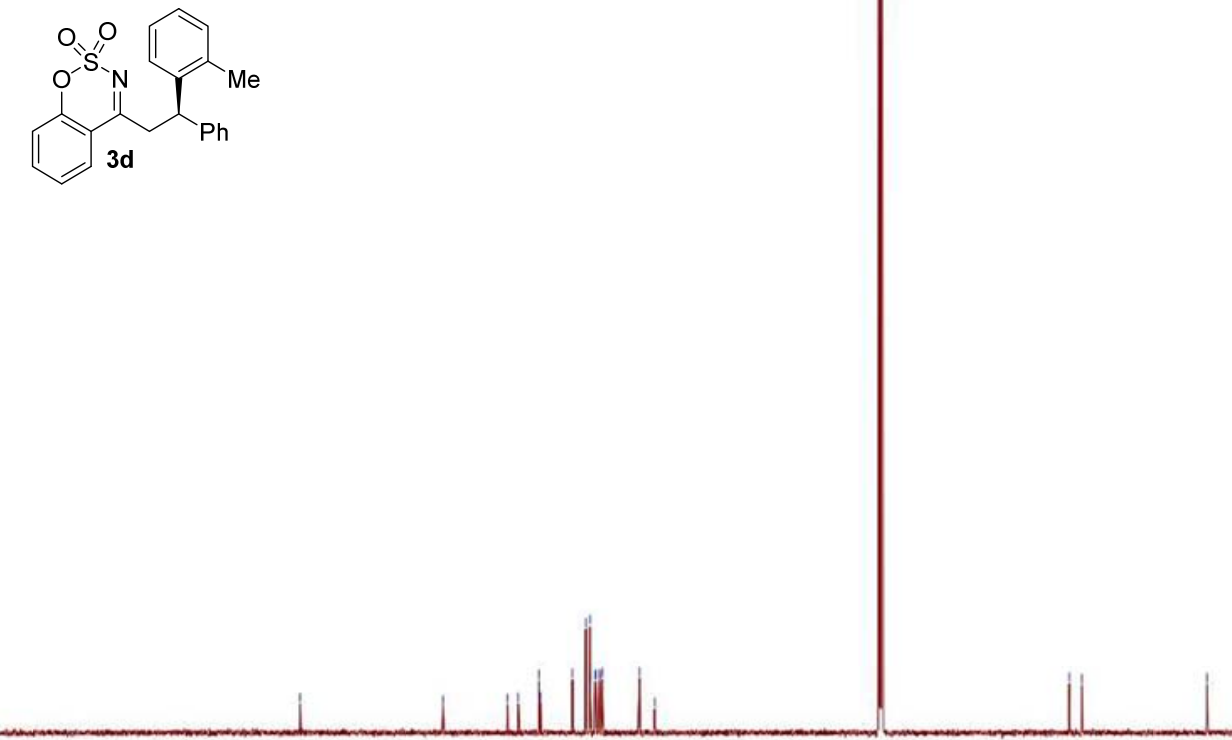

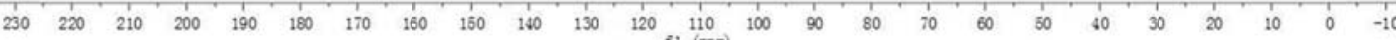



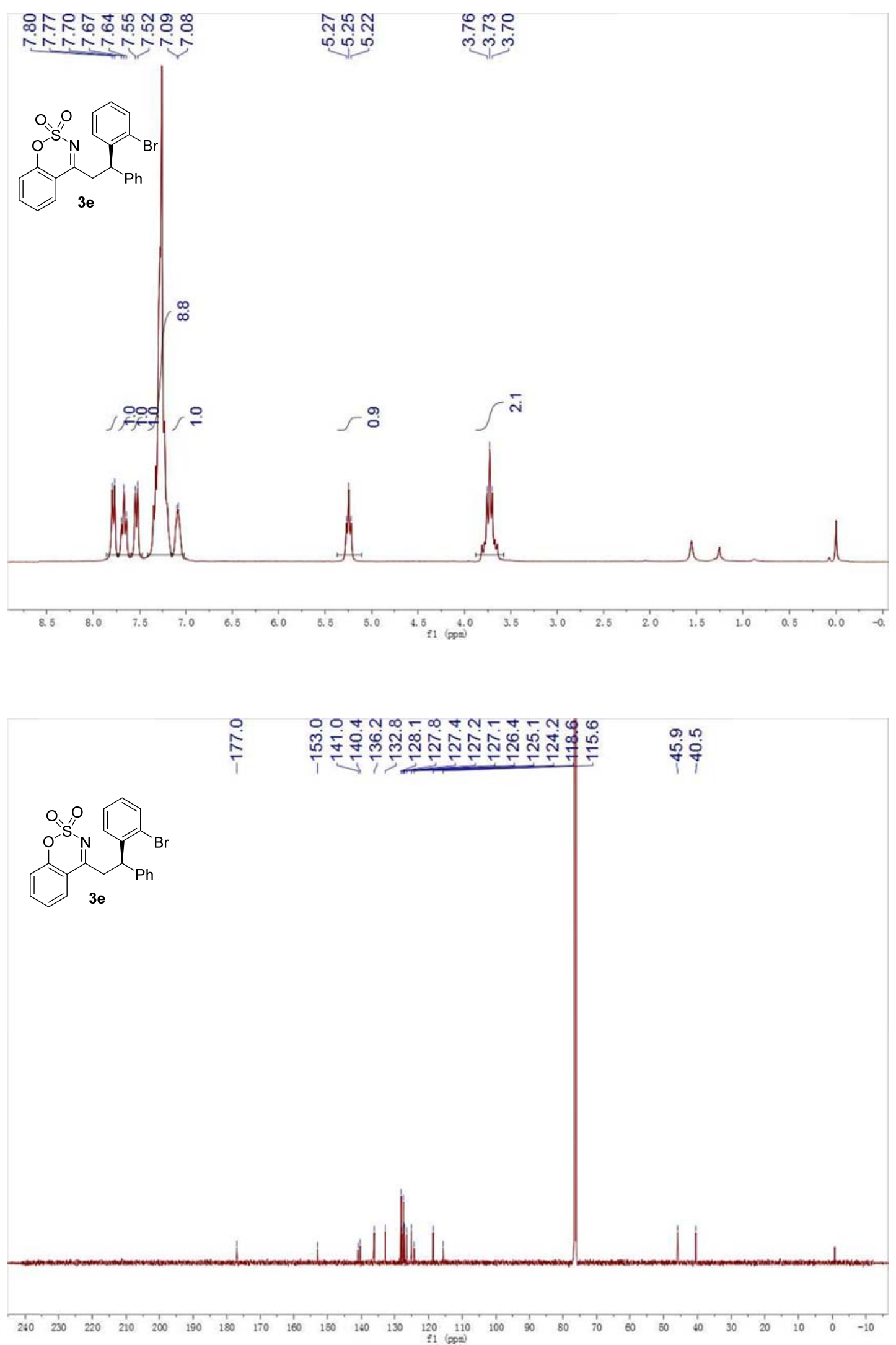


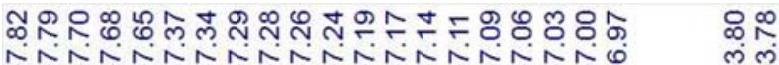

NNNNNNNNNNNNNNNNNO
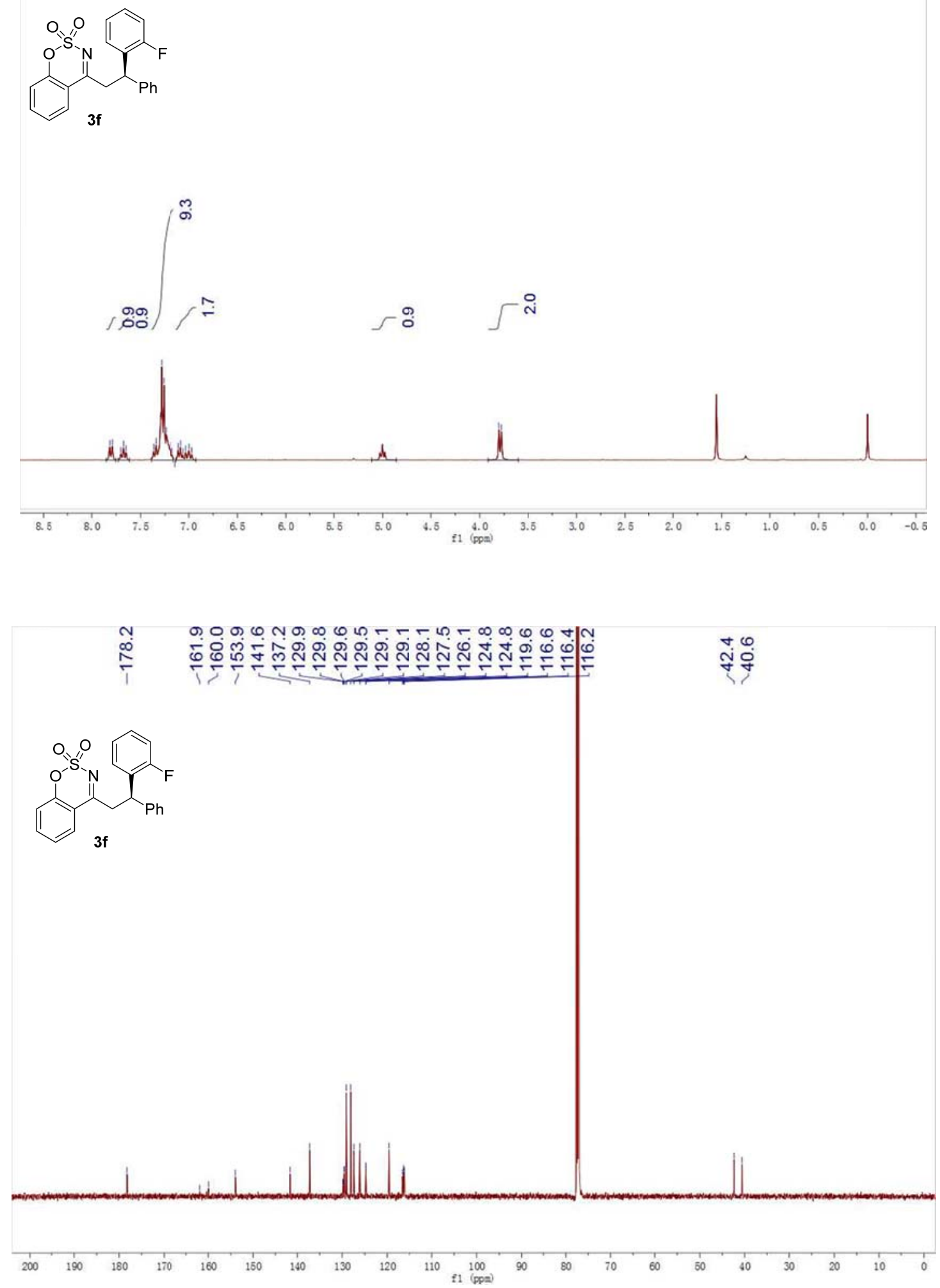


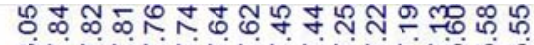

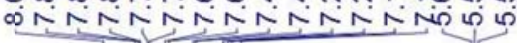

(19

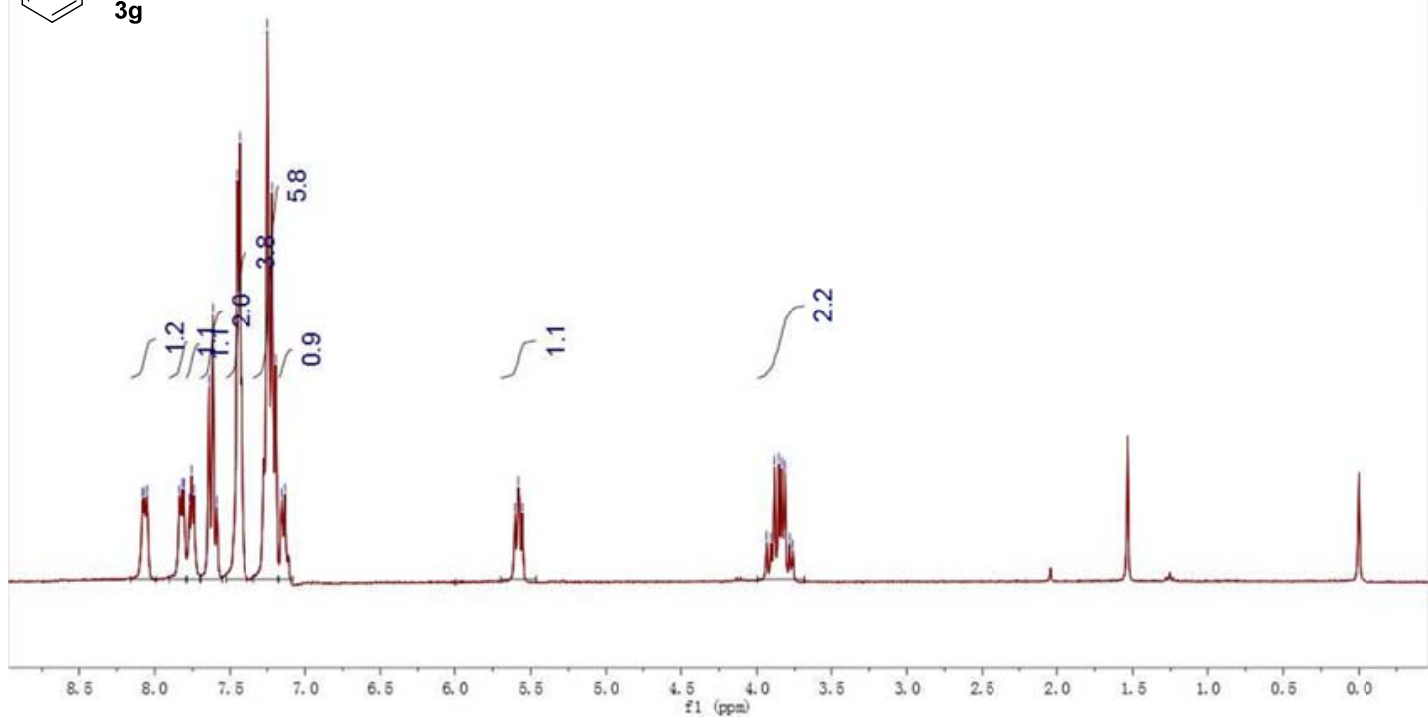

$\frac{1}{1}$

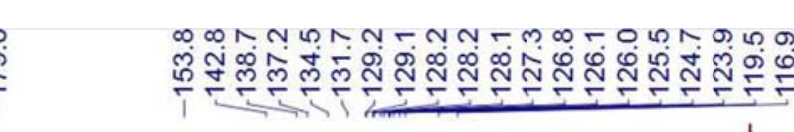

至

这䖟

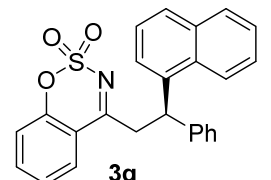

$3 \mathrm{~g}$

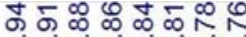

लेल लिल ले
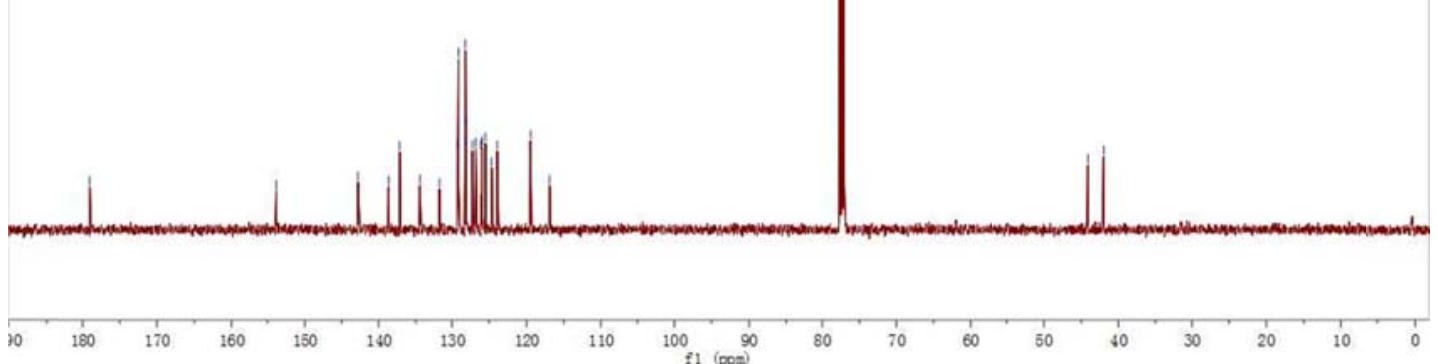

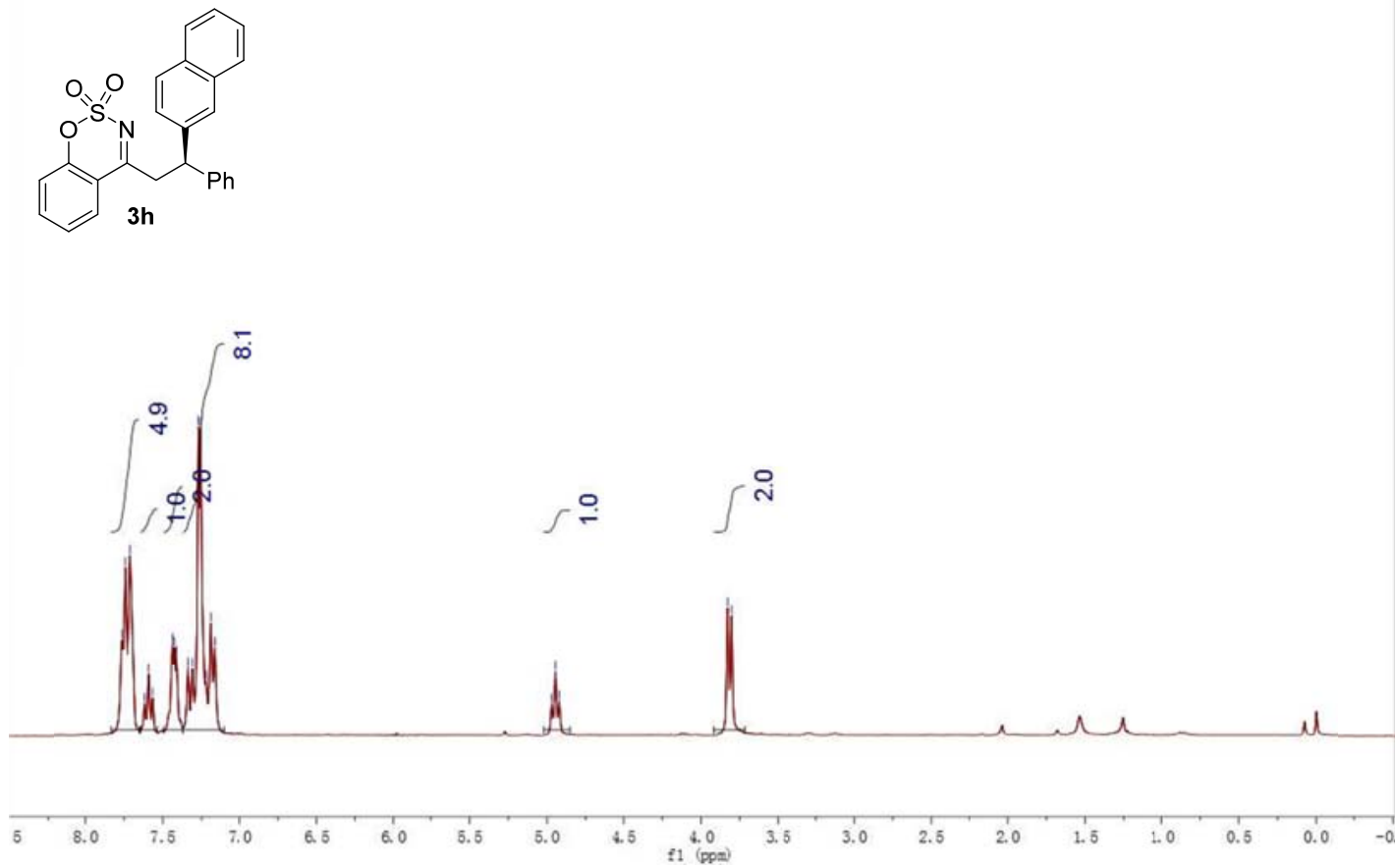

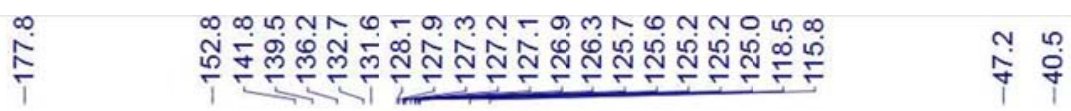
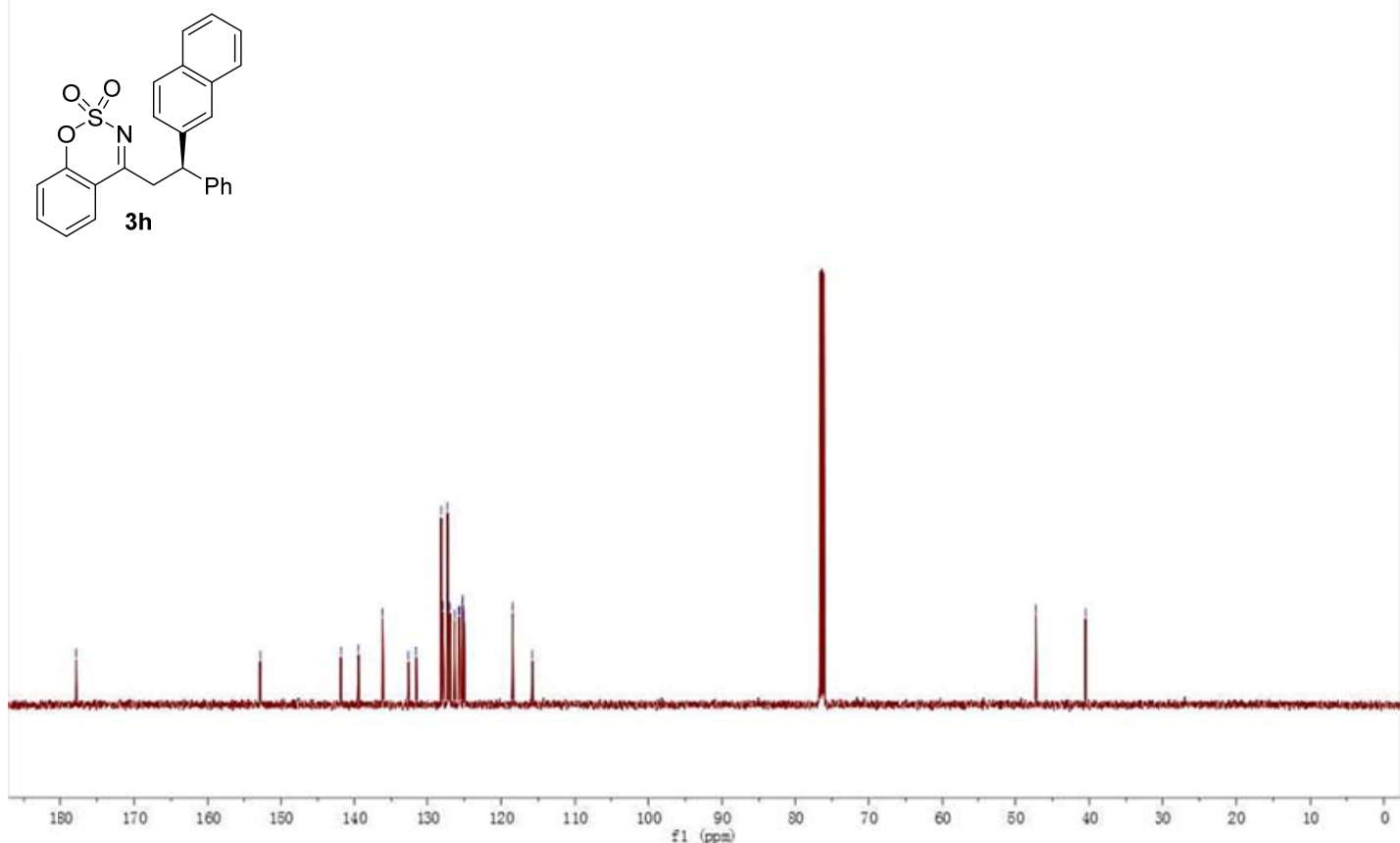


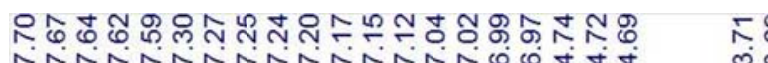

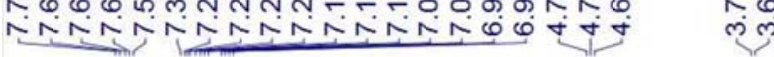

$\stackrel{\text { }}{\uparrow}$<smiles></smiles>

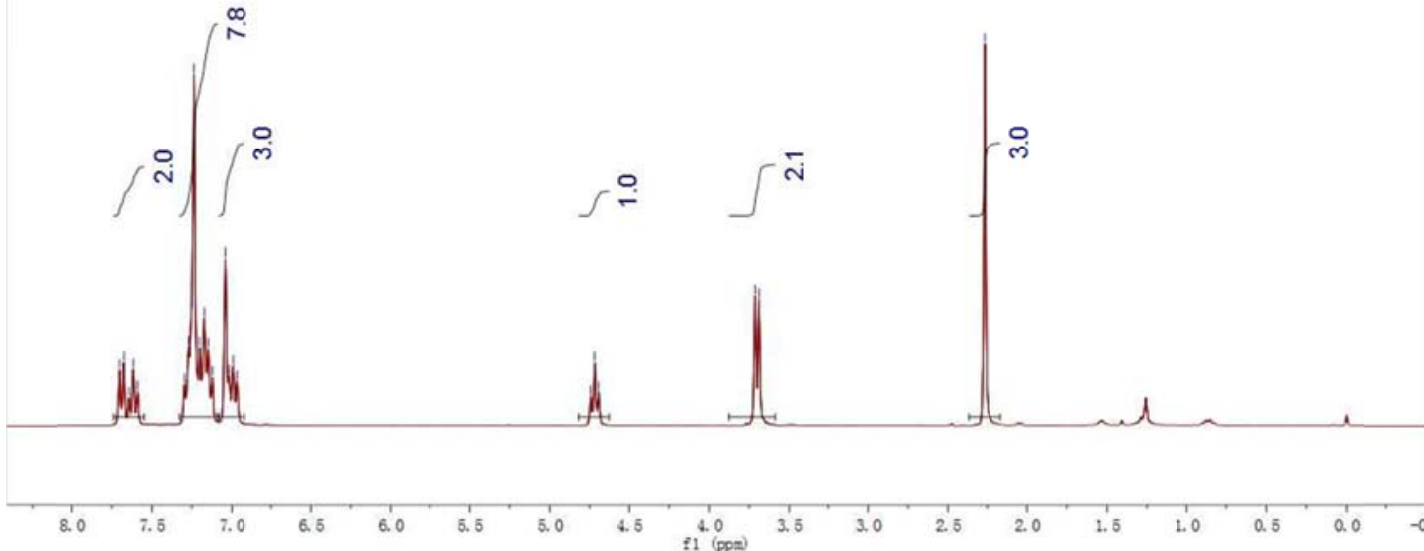

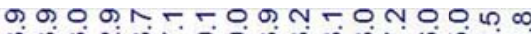

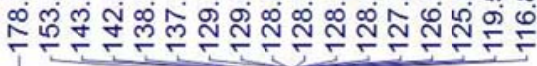

$\stackrel{m}{\infty} \stackrel{\infty}{\infty} \underset{j}{\infty} \stackrel{\infty}{\leftarrow}$
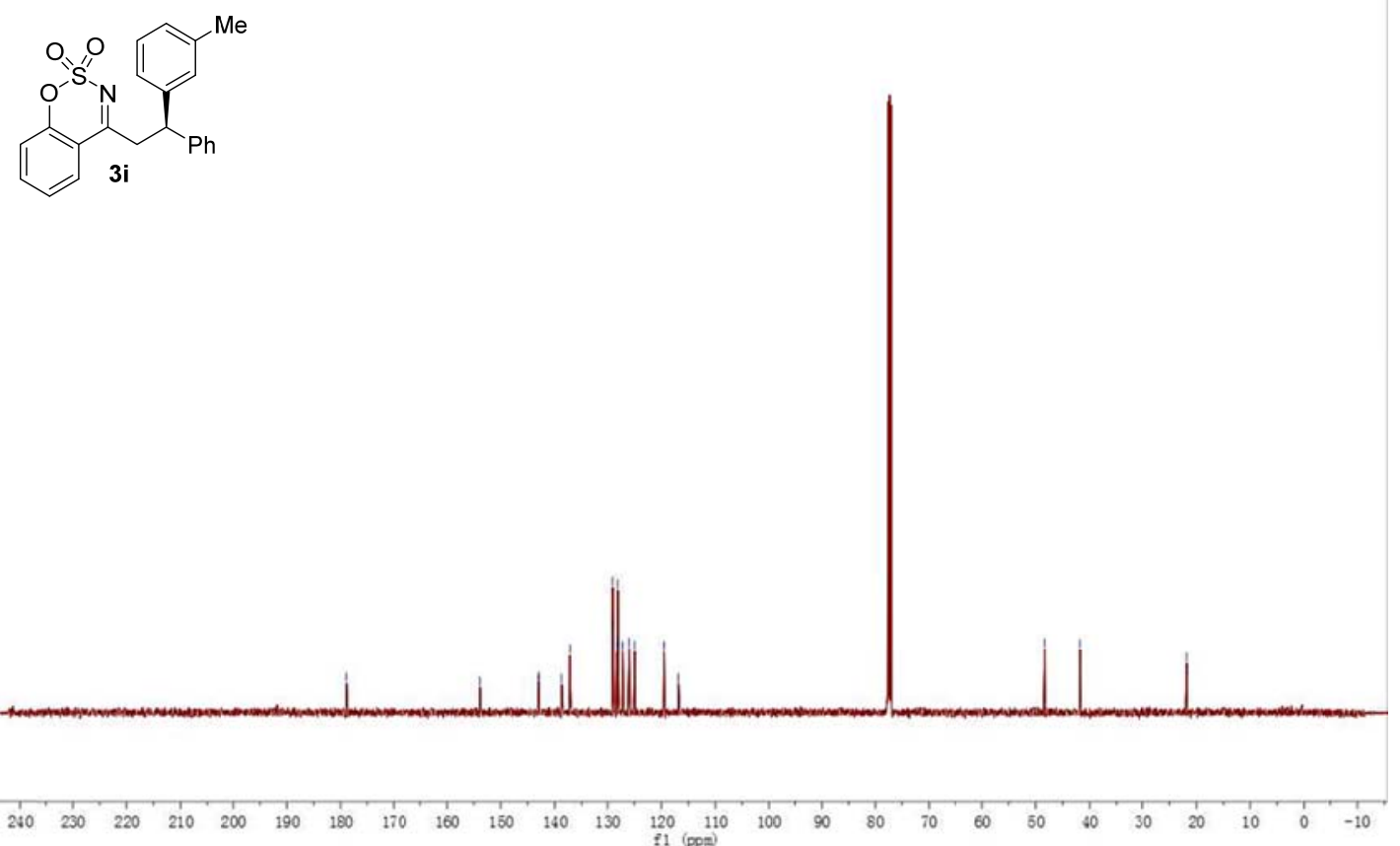


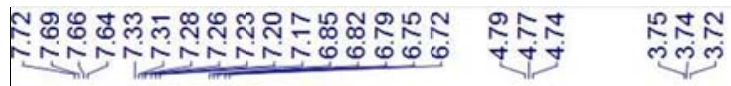
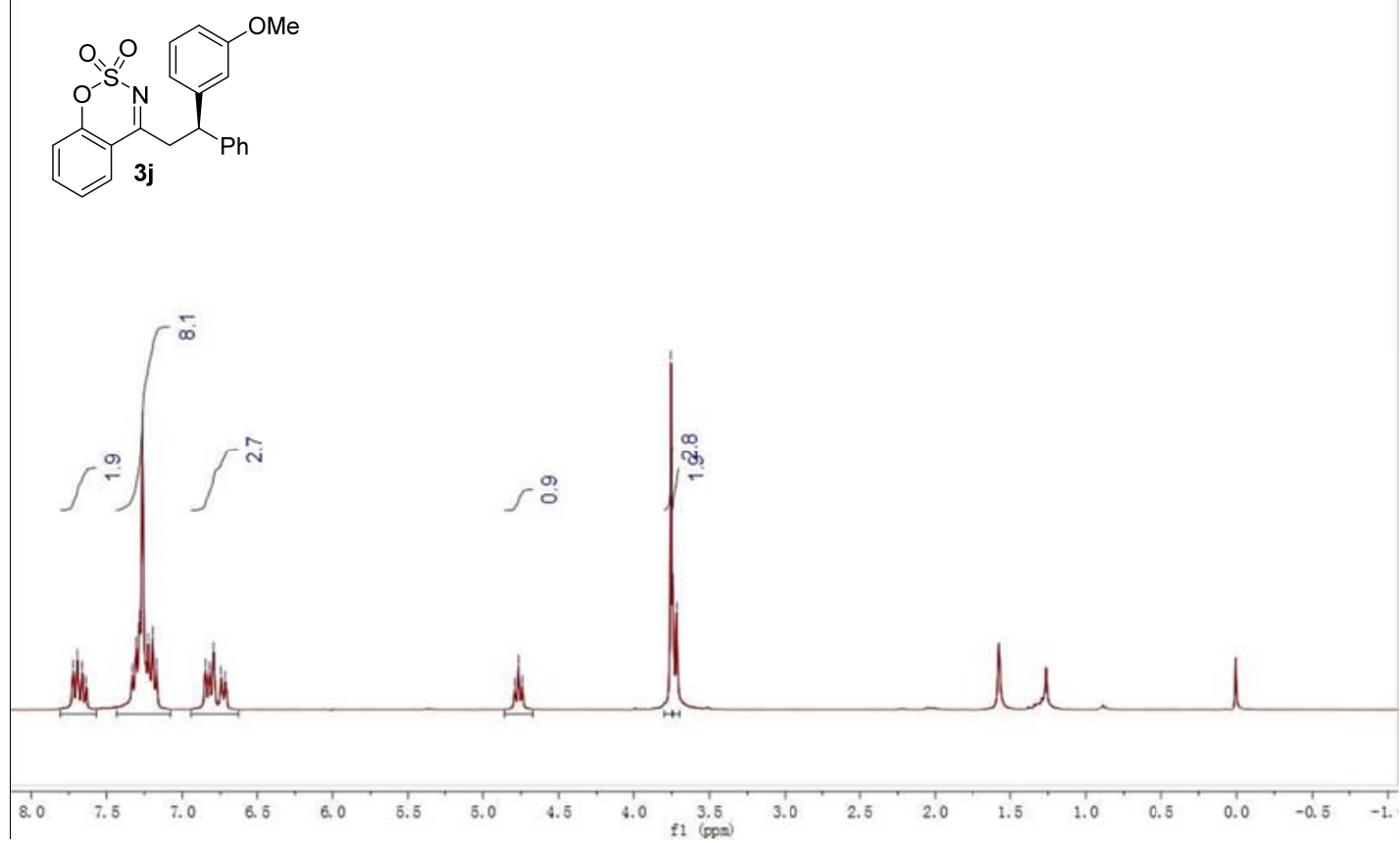

$\begin{array}{ll}+ & 0 \\ \infty & 0\end{array}$

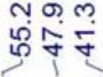<smiles>COc1cccc(C(CC2=NS(=O)(=O)Oc3ccccc32)c2ccccc2)c1</smiles>
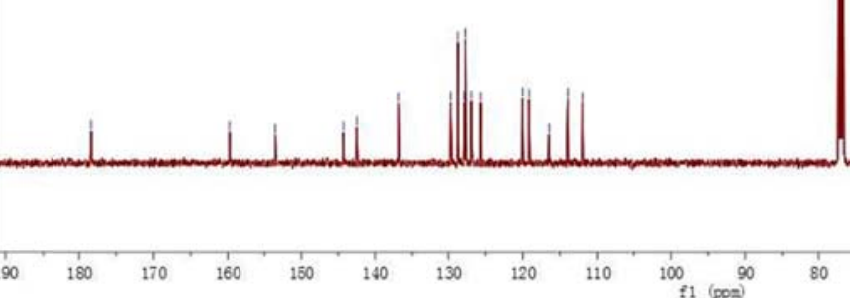


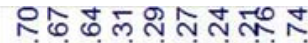

NNNNNNNO<smiles>COc1ccc([C@H](CC2=NS(=O)(=O)Oc3ccccc32)c2ccccc2)cc1OC</smiles>
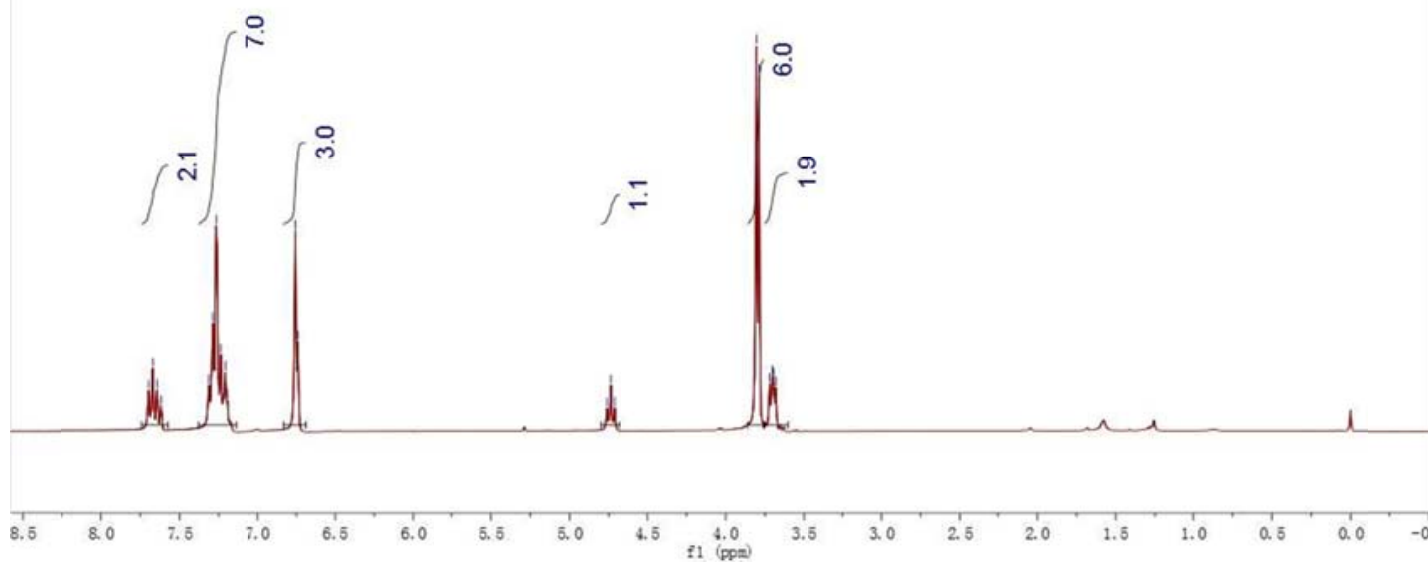

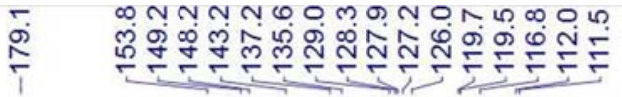

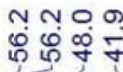
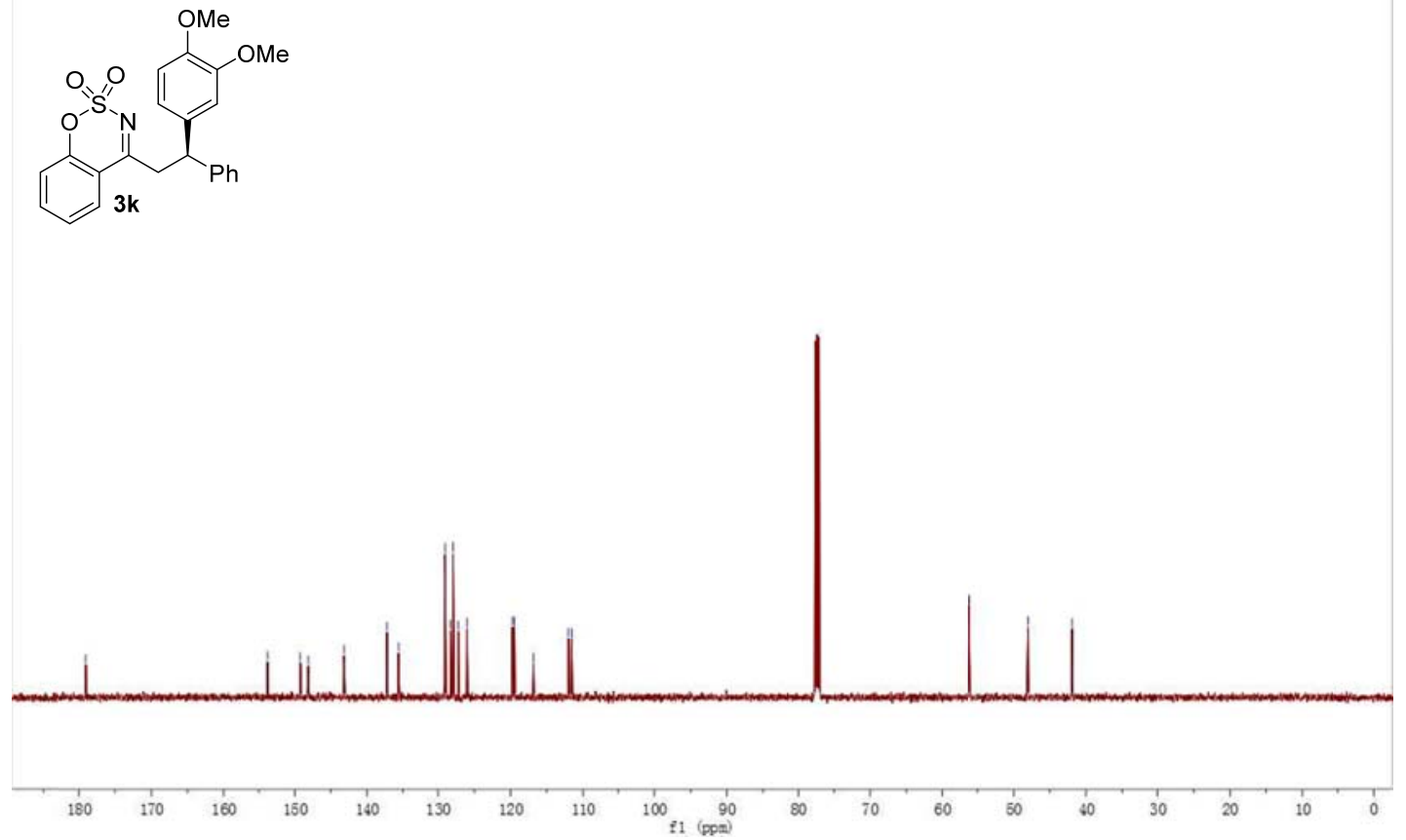
ద

NNNNNNNNNNNNNin
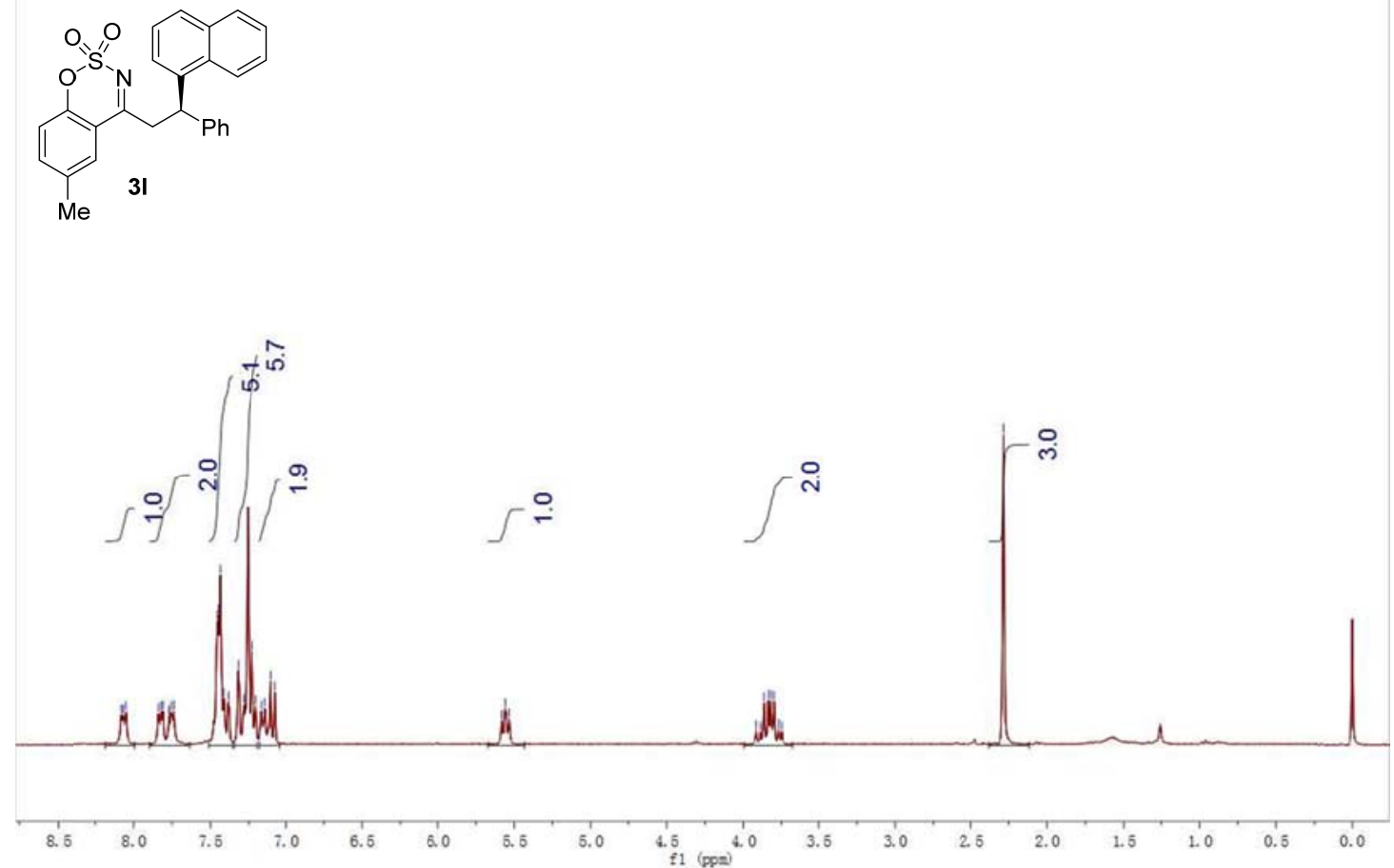

$\frac{\text { N }}{\mathrm{N}}$

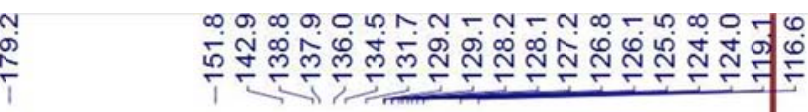

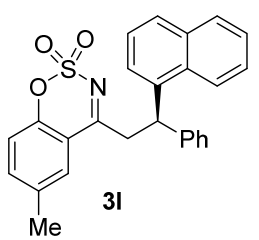

$\mathrm{Me}$

ঙ্ं

$\underset{\sim}{\grave{1}}$

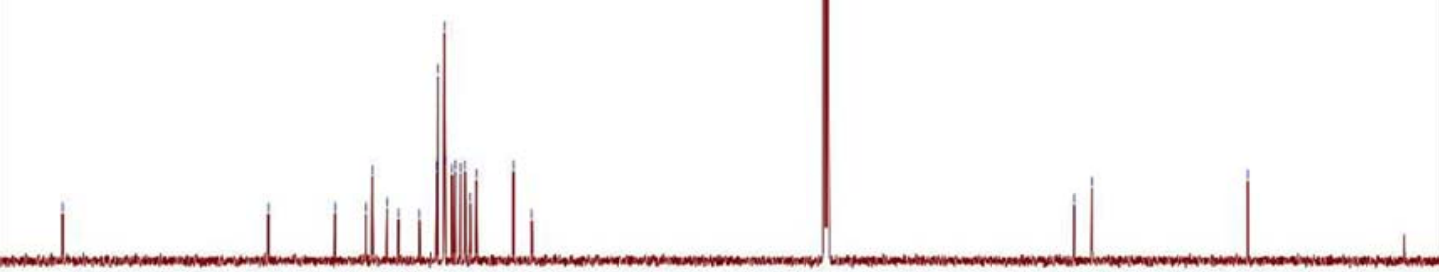

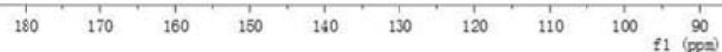



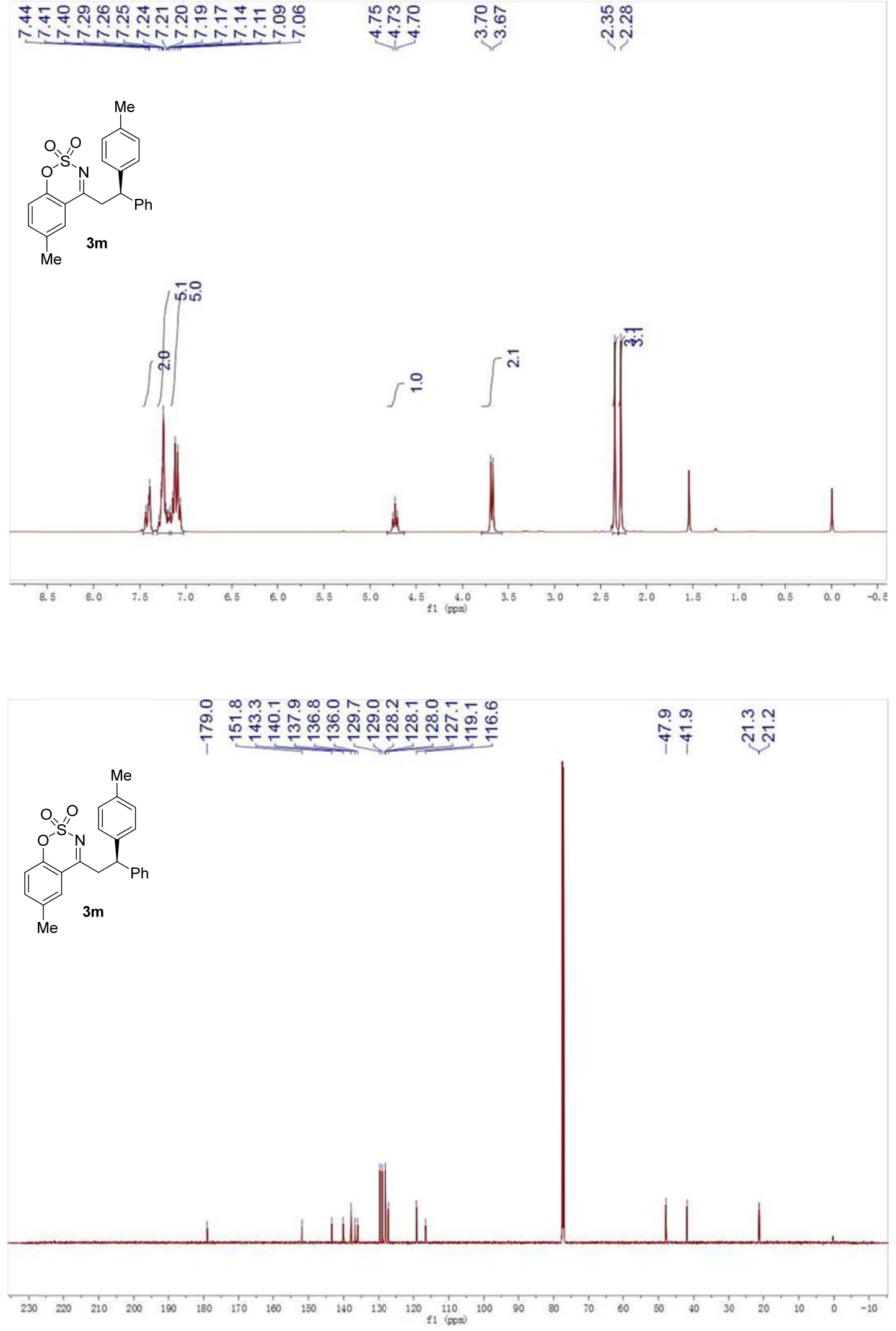

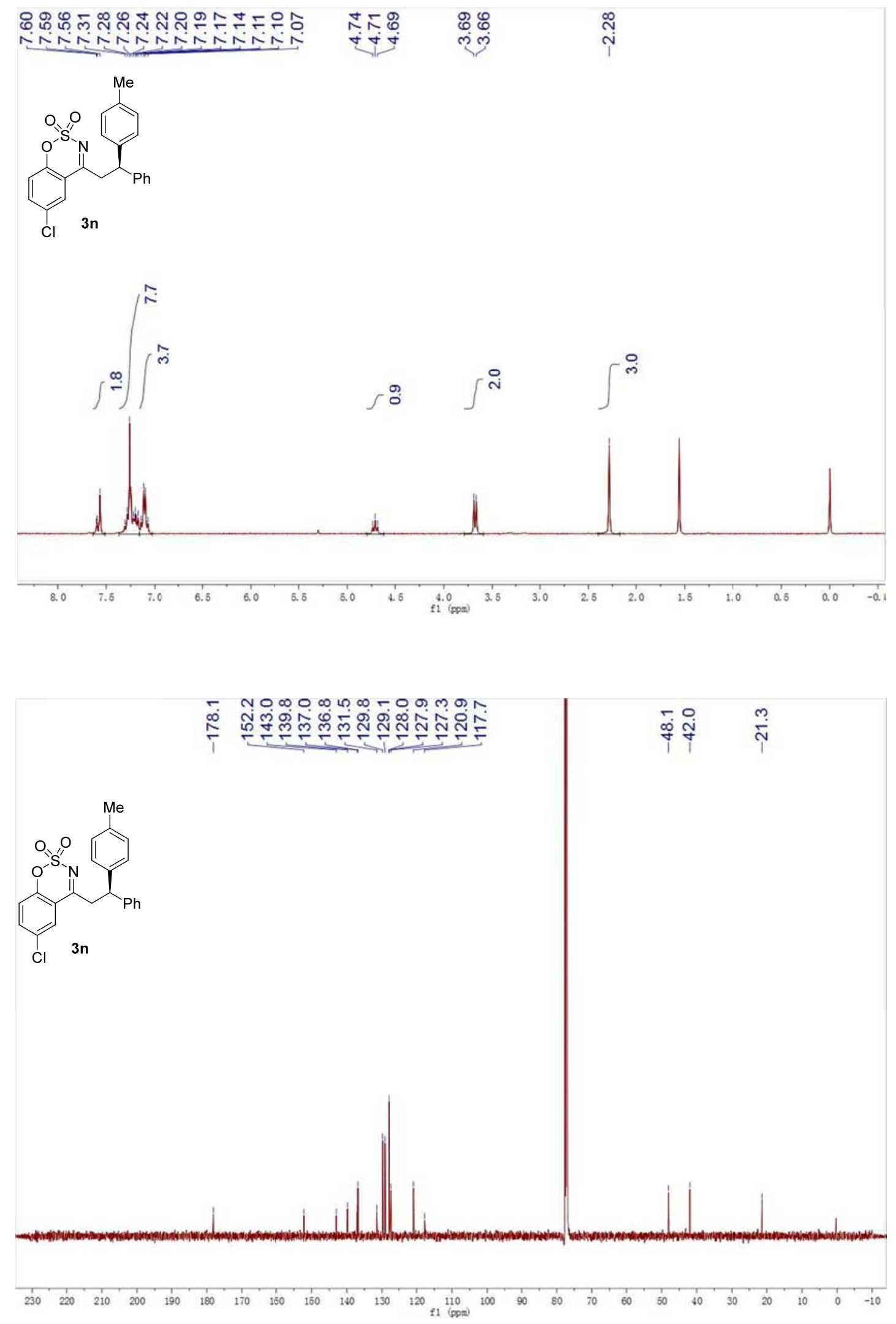


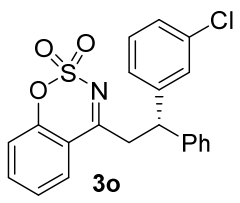

$$
\left.\Gamma \stackrel{i}{i}\right|_{\infty} ^{\infty}
$$<smiles>CCCCCCCCCCCC</smiles>
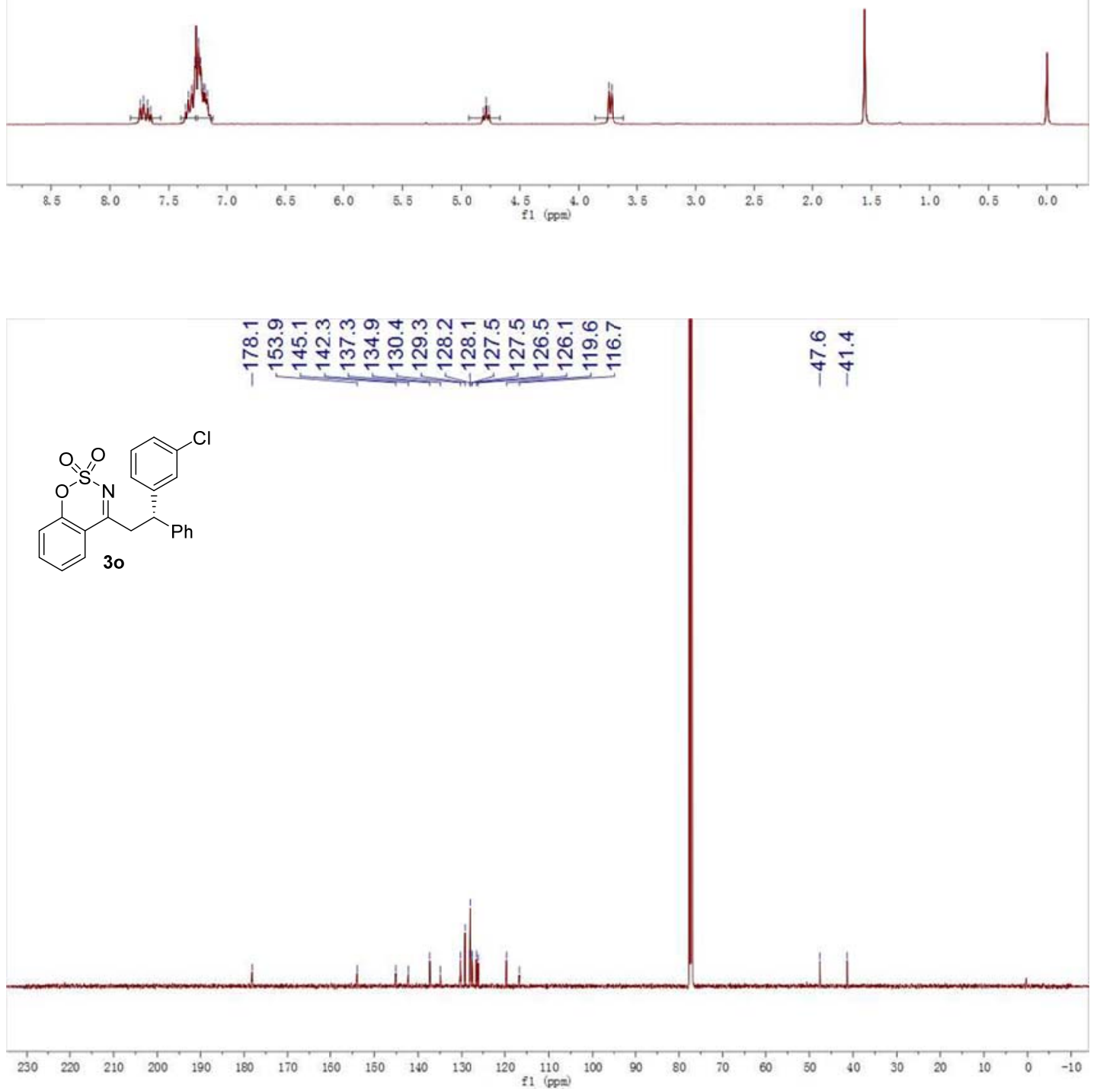


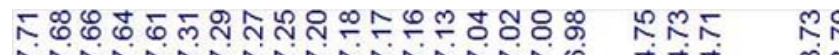<smiles>Cc1cccc(C(CC2=NS(=O)(=O)Oc3ccccc32)c2ccccc2)c1</smiles>
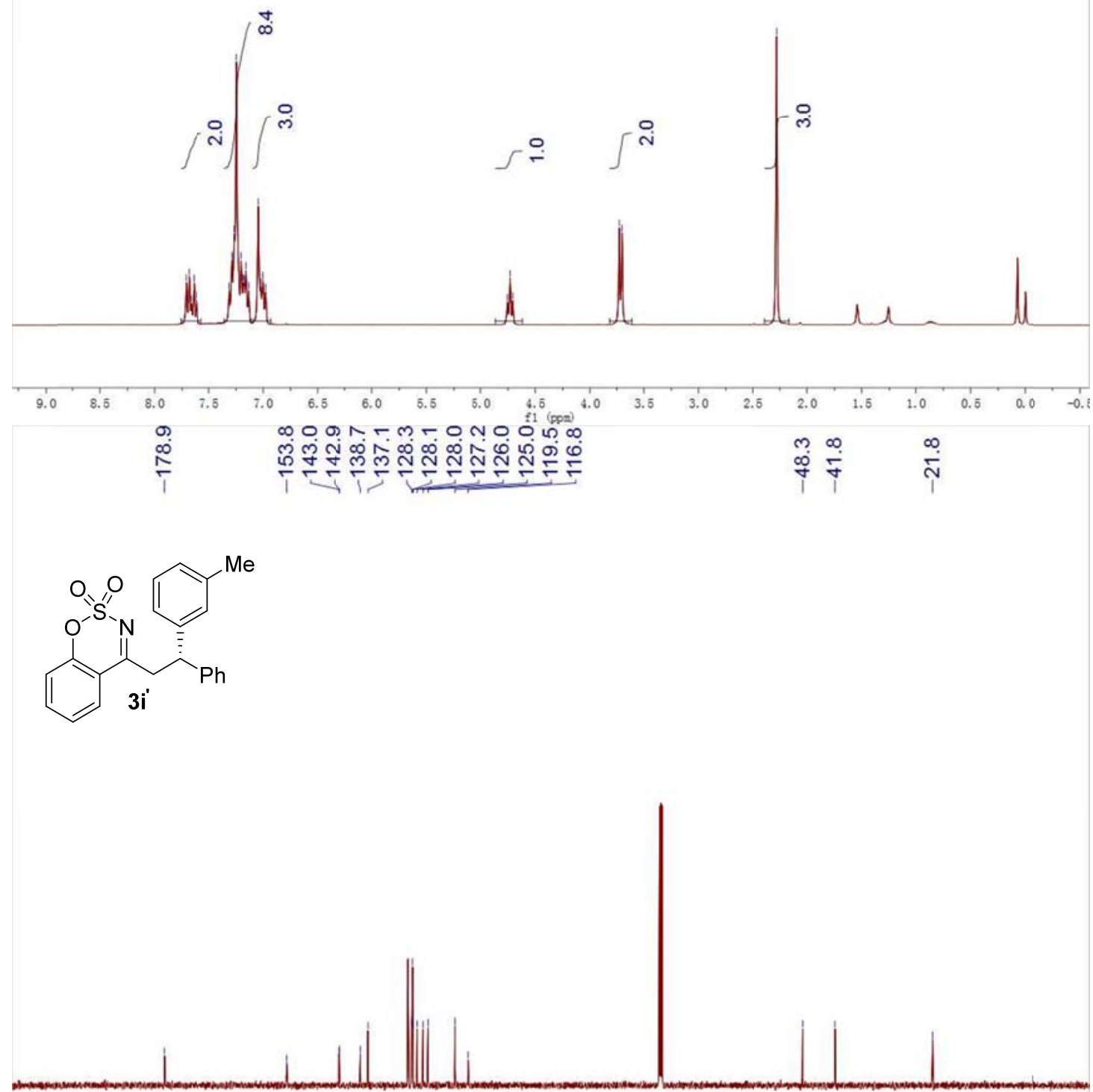

$\begin{array}{lllllllll}200 & 190 & 180 & 170 & 160 & 150 & 140 & 130 & 100\end{array}$ $110 \quad 100$ 


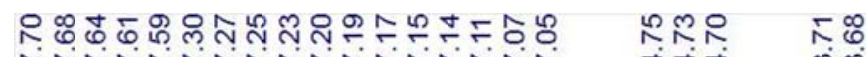

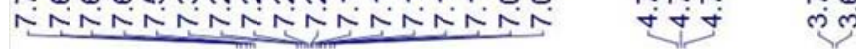

$\stackrel{\leftrightarrow}{\text { N }}$<smiles>Cc1ccc(C(Cc2ccccc2OS(=O)(=O)O)c2ccccc2)cc1</smiles>

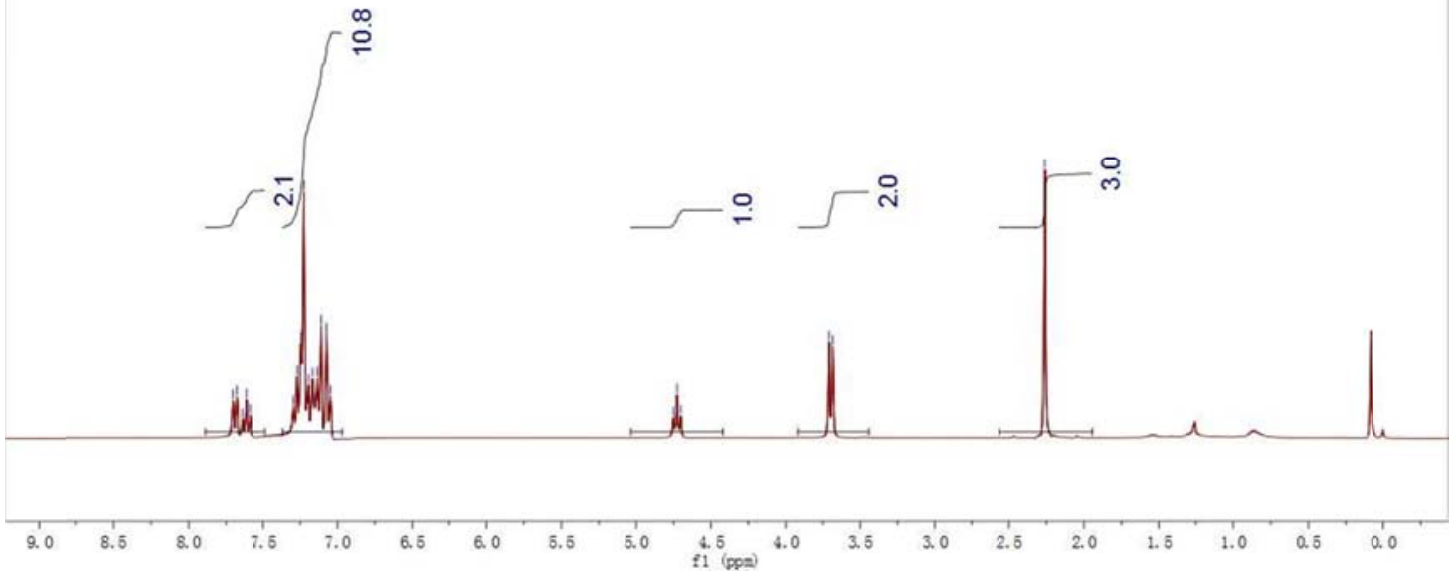

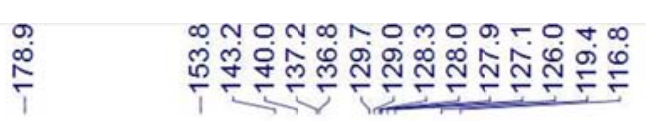

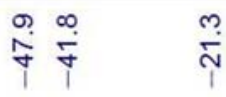
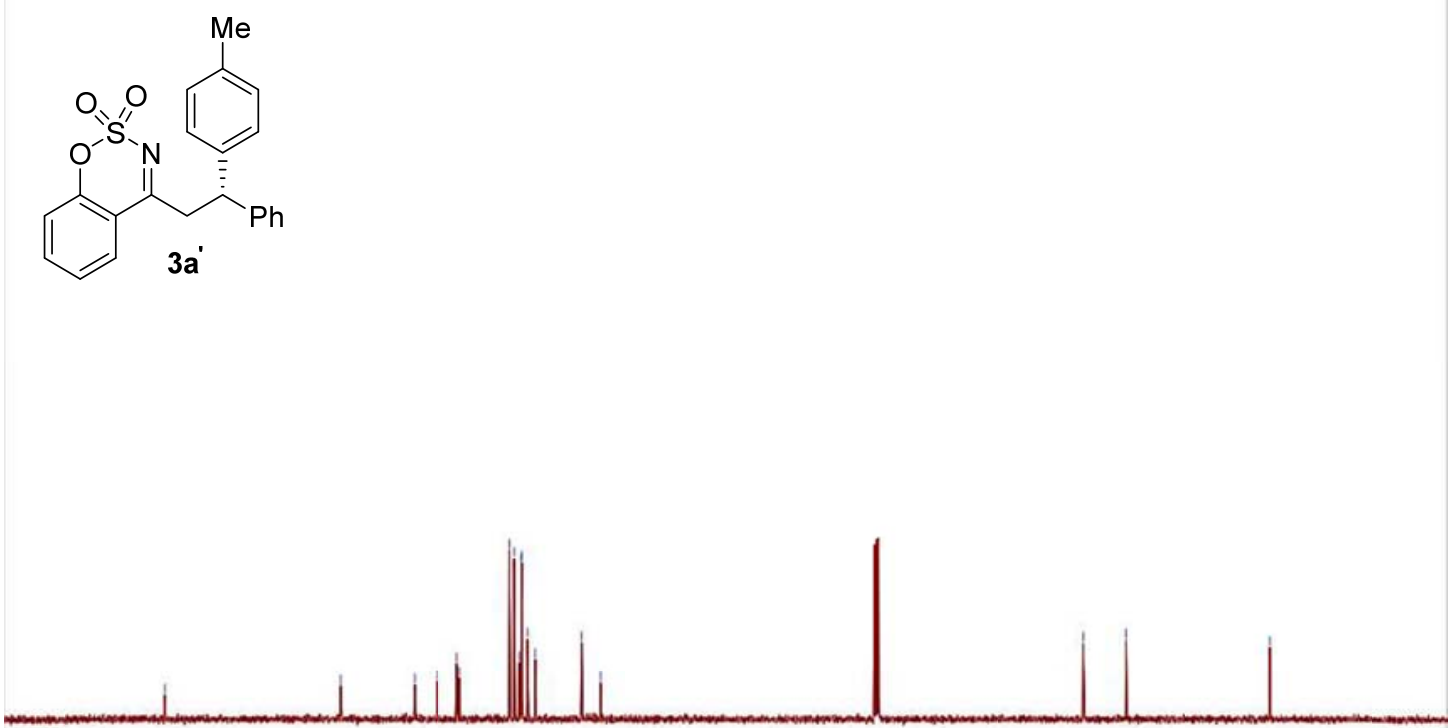

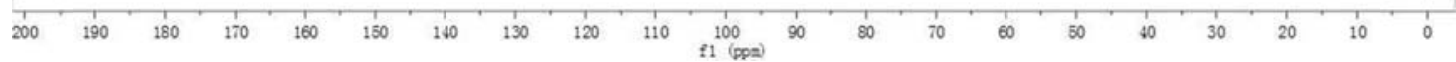



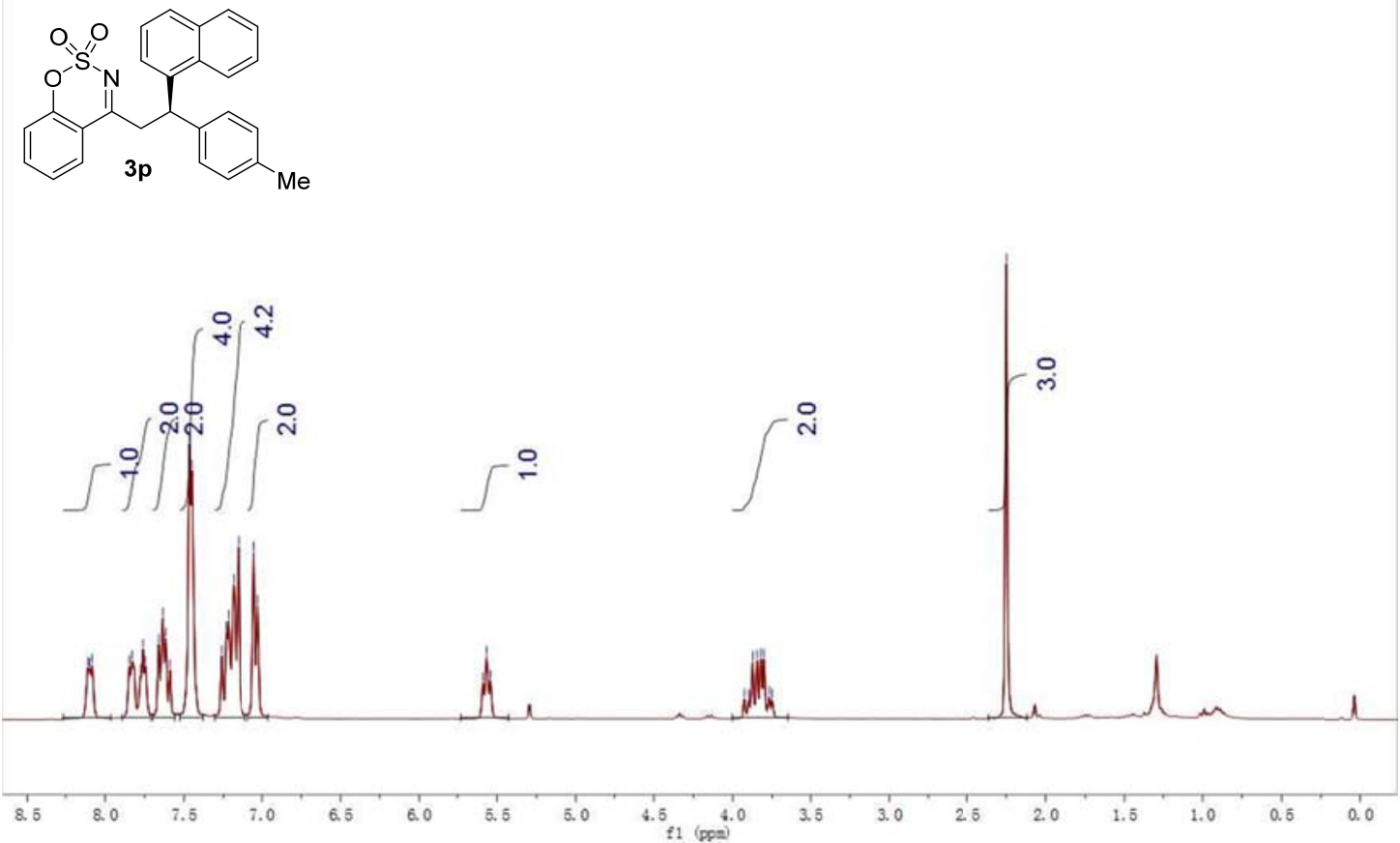

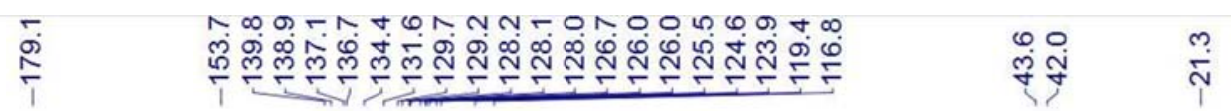<smiles>Cc1ccc(C(CC2=NS(=O)(=O)Oc3ccccc32)c2cccc3ccccc23)cc1</smiles>
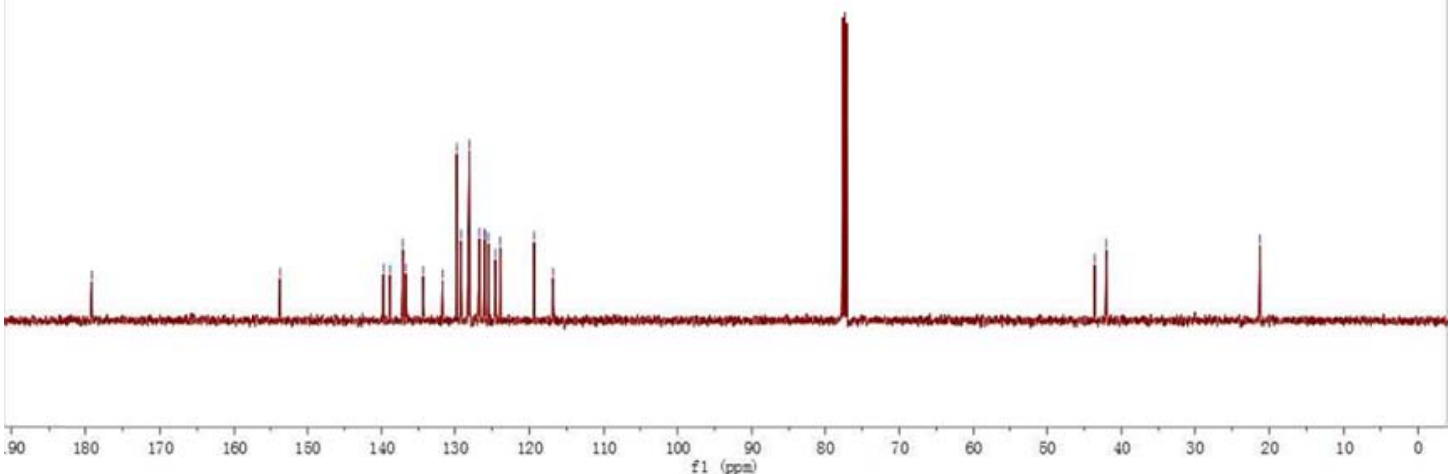


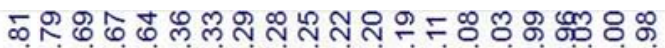

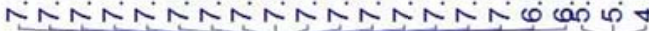

宓企

ตุ

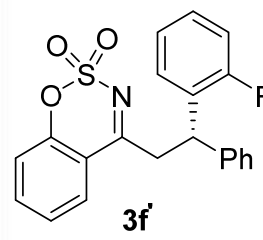

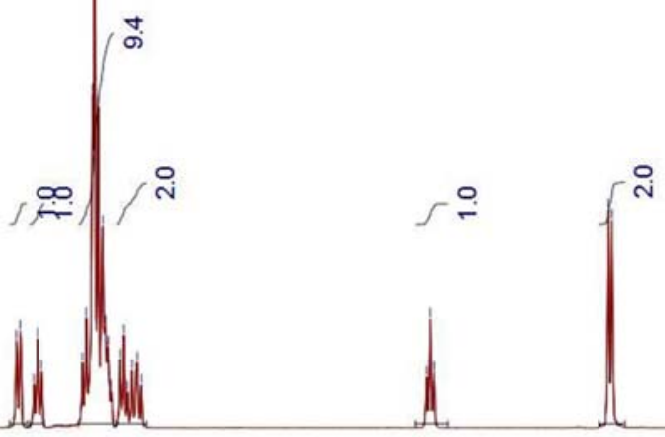

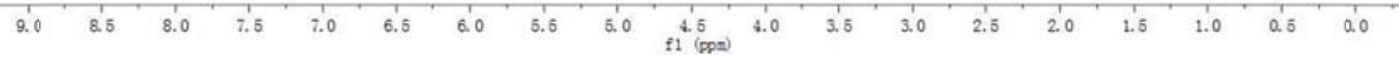

m $\quad$ ต

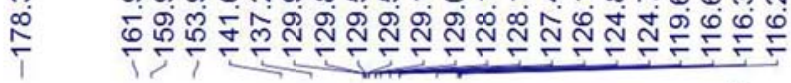

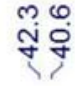
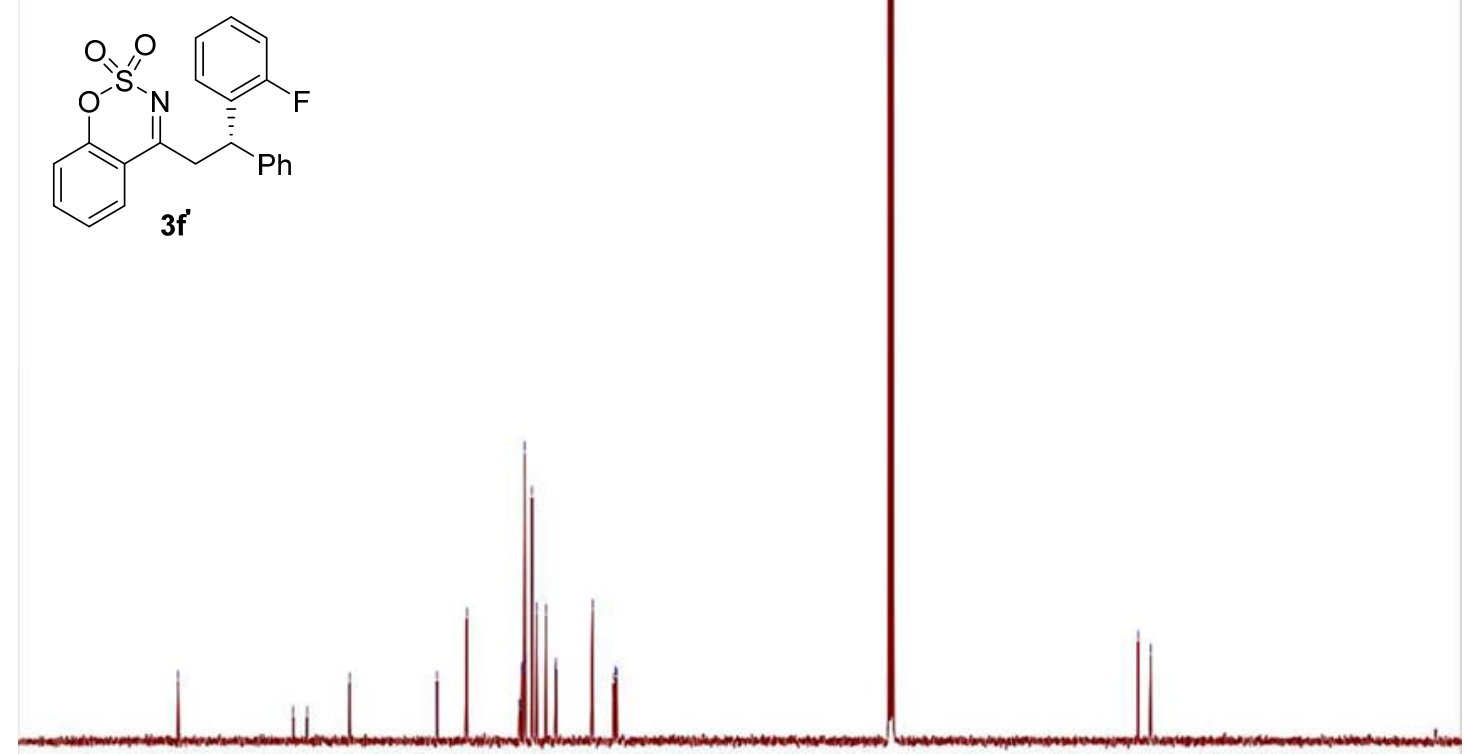

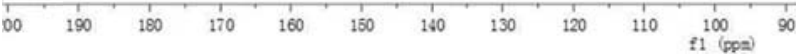



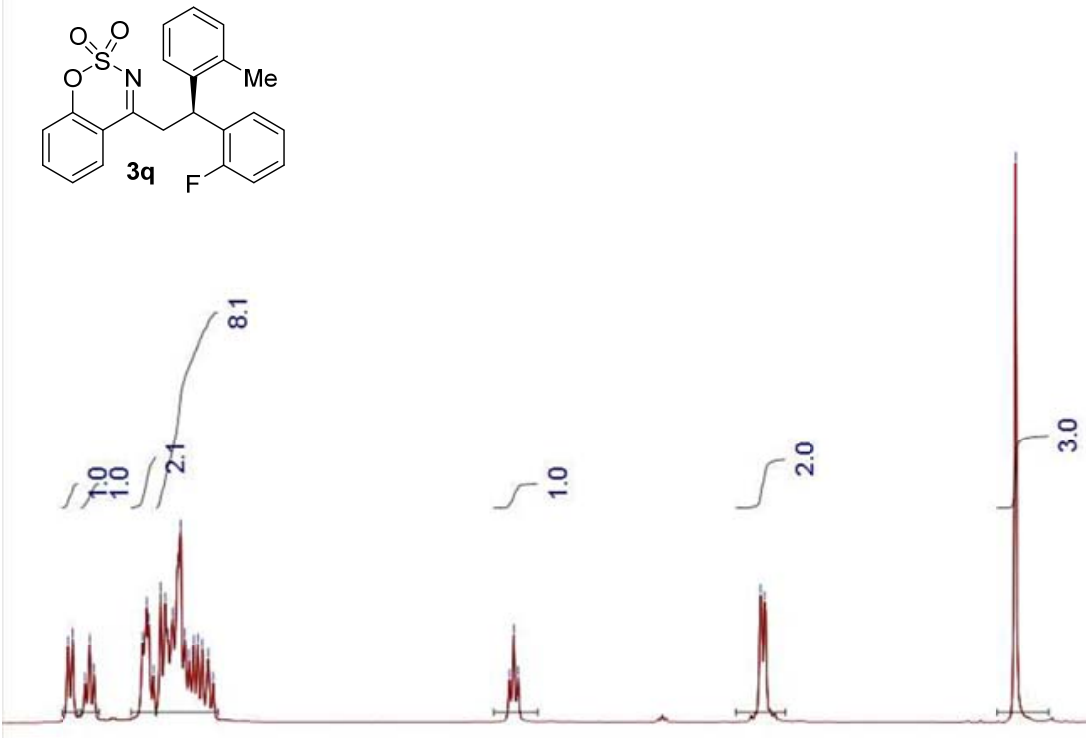

8. 0

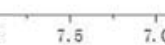

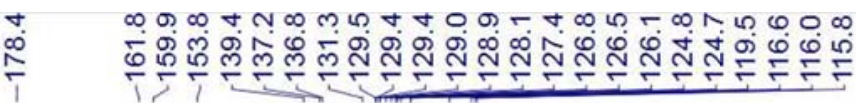

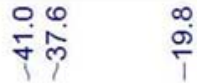
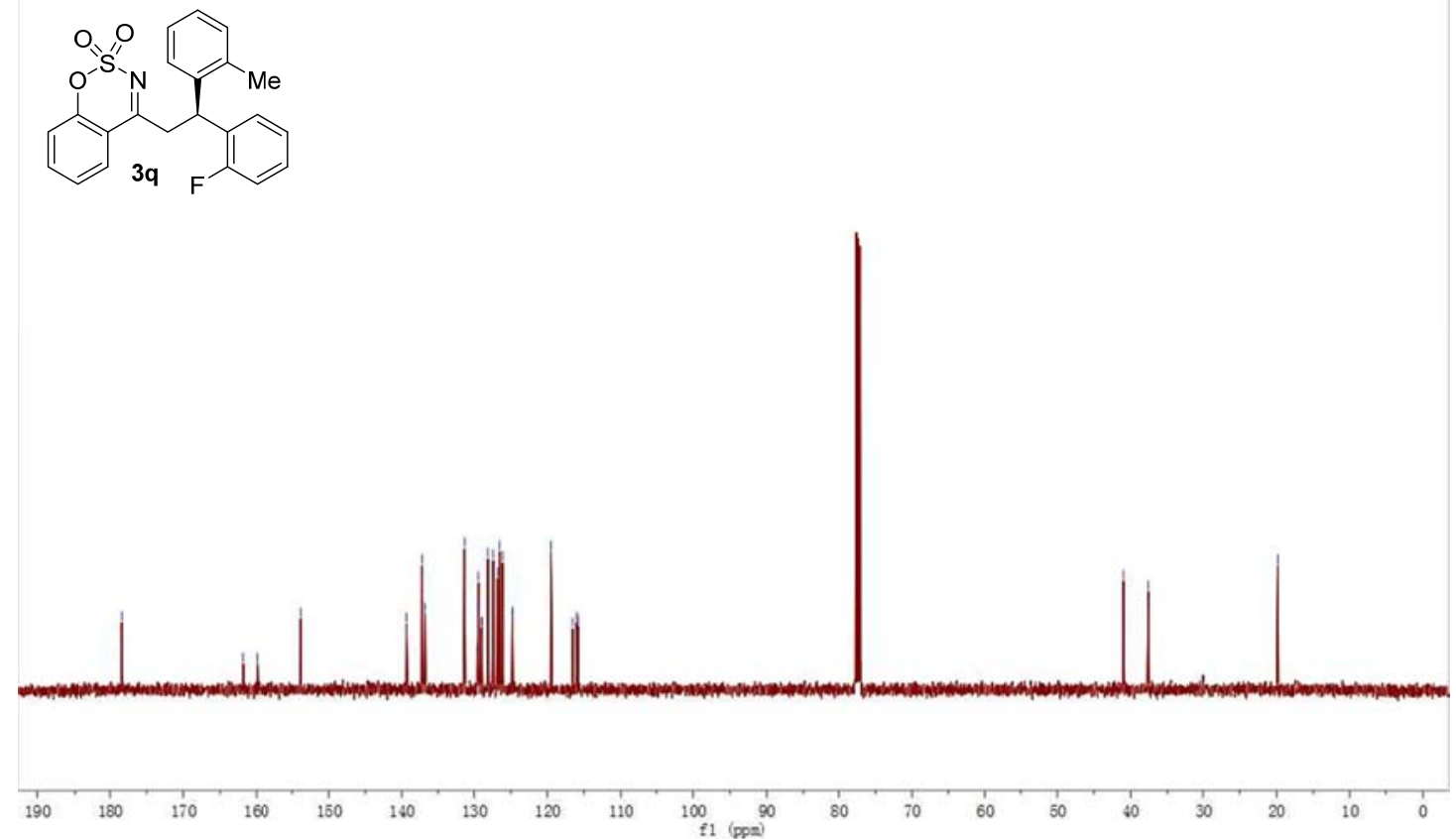


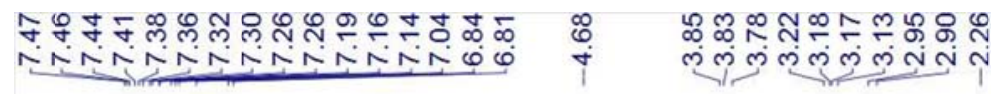<smiles>COc1ccc(CC(NS(=O)(=O)Oc2ccccc2)(c2ccccc2)c2ccc(C)cc2)cc1</smiles>
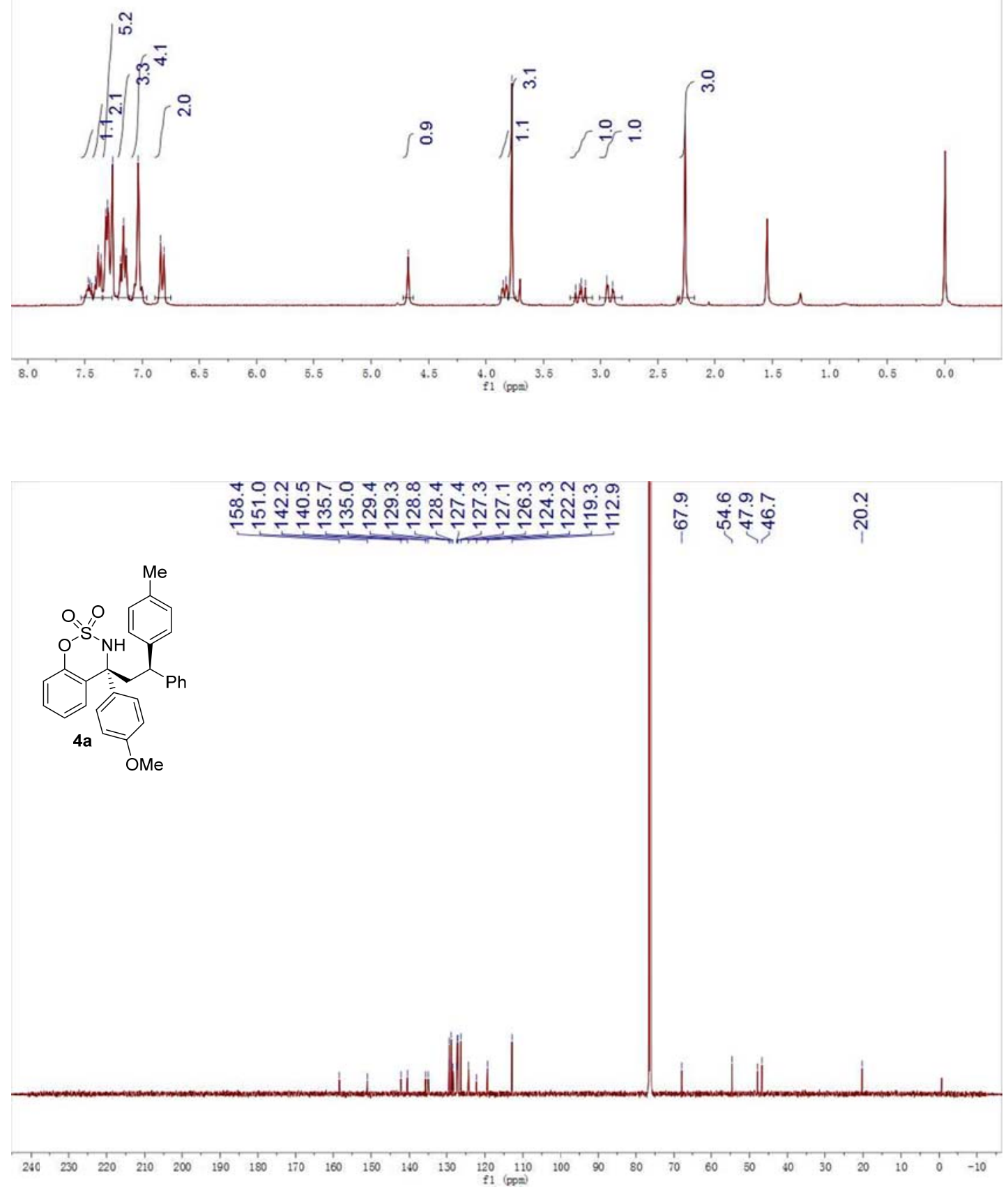


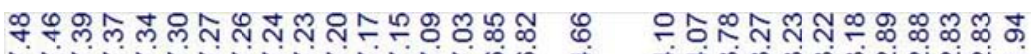

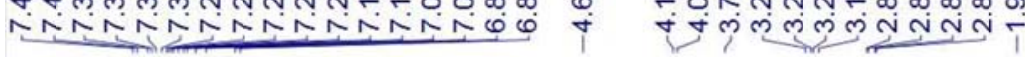
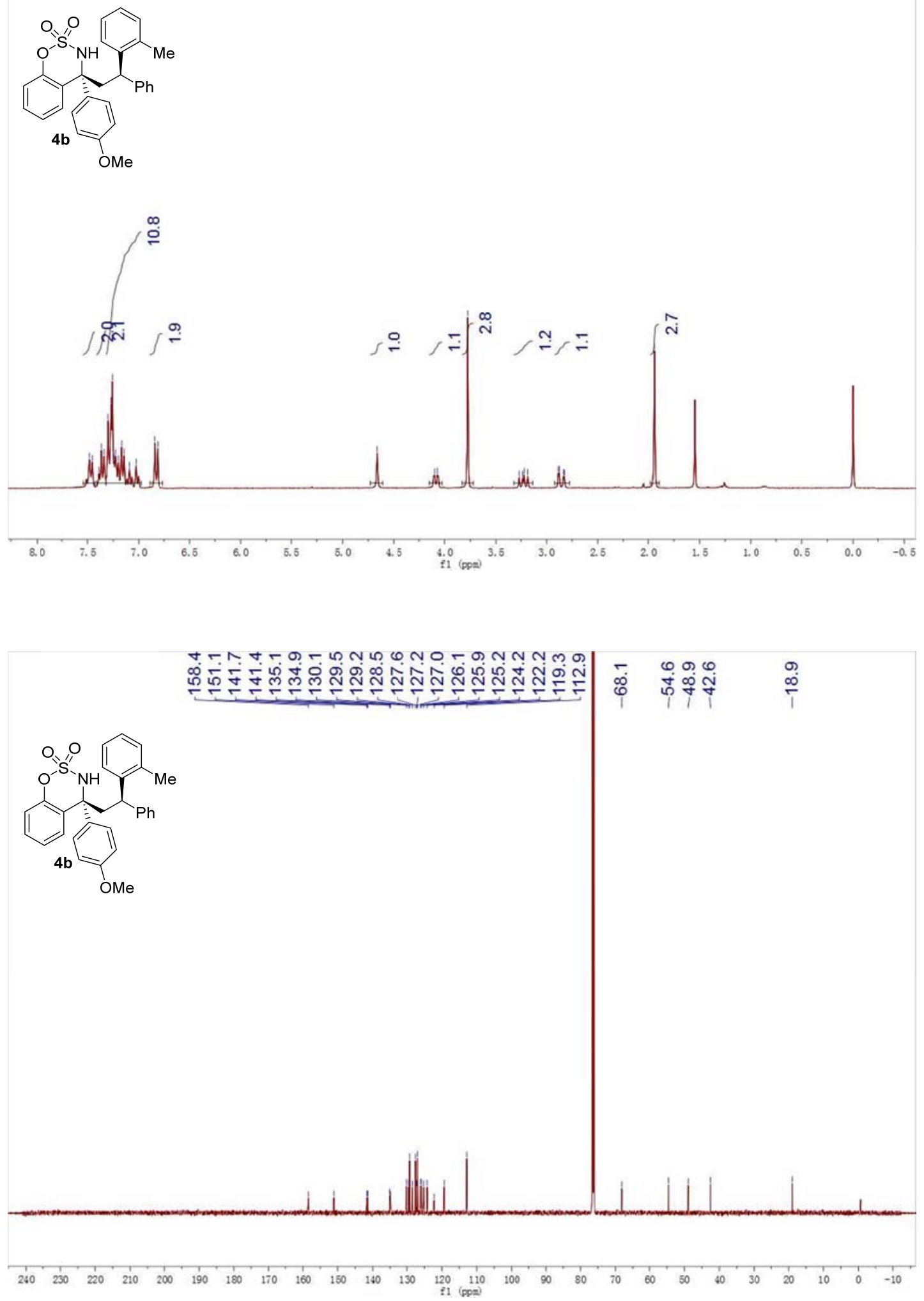


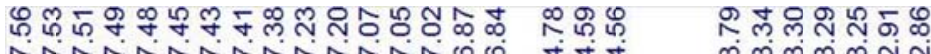

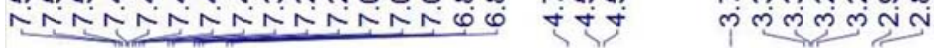<smiles>COc1ccc(C(c2ccccc2Br)c2ccccc2OS(=O)(=O)O)cc1</smiles>
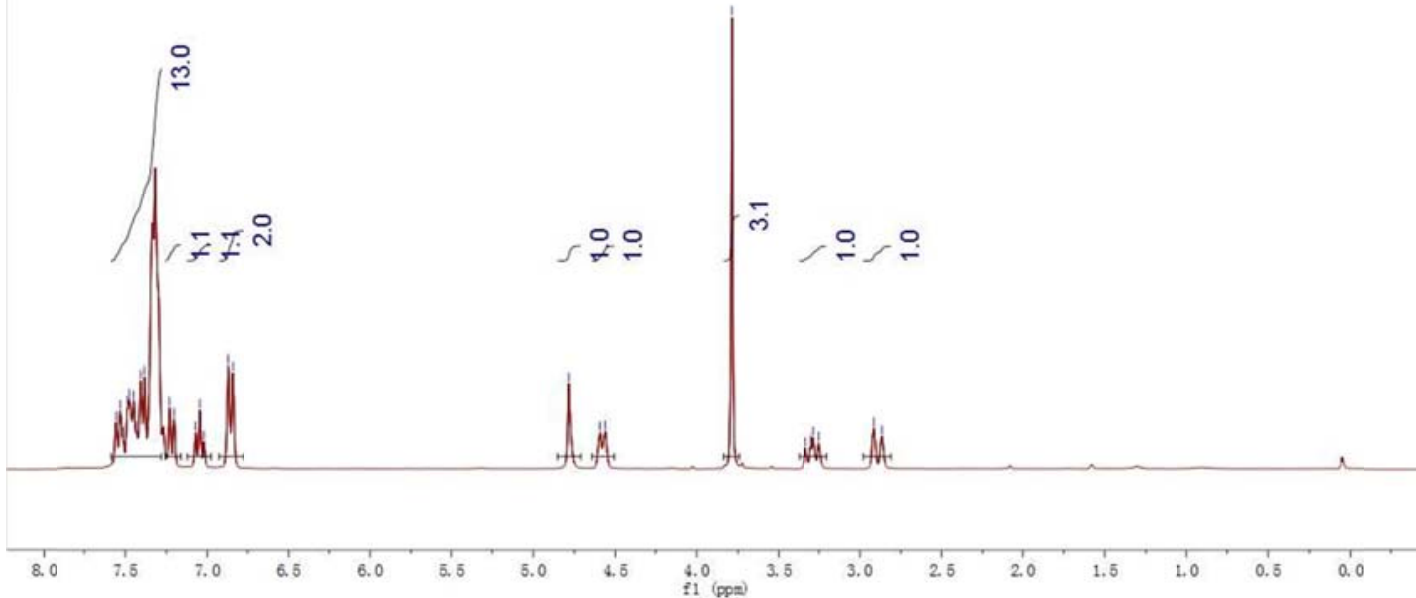

蕰

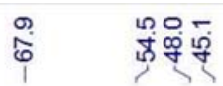
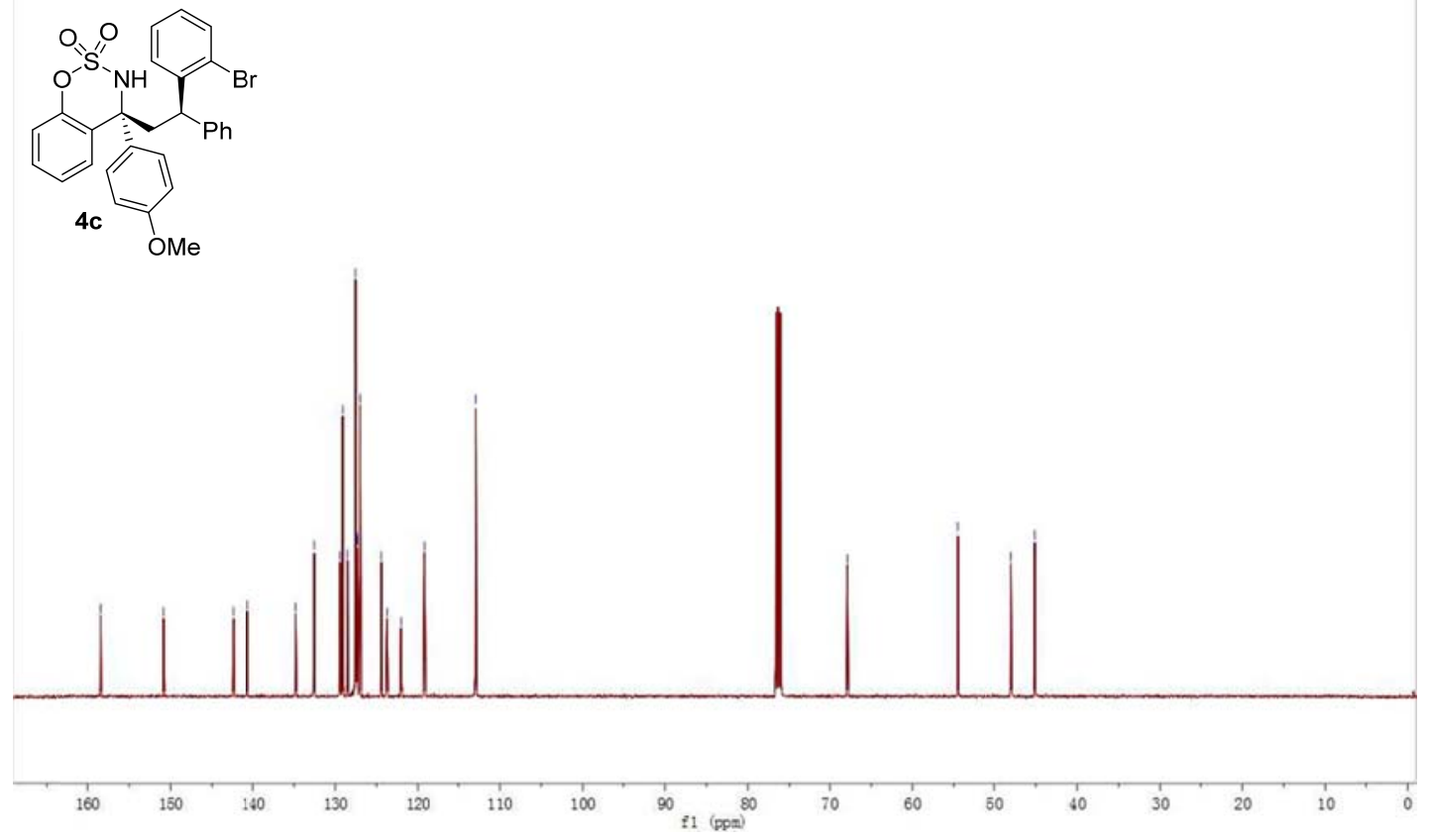


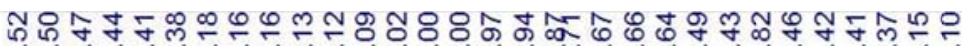

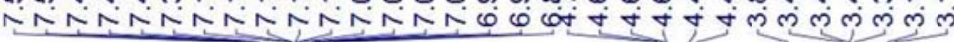<smiles>COc1ccc([C@H]2c3ccccc3CN(S(=O)(=O)O)C23Oc2ccccc23)cc1</smiles>

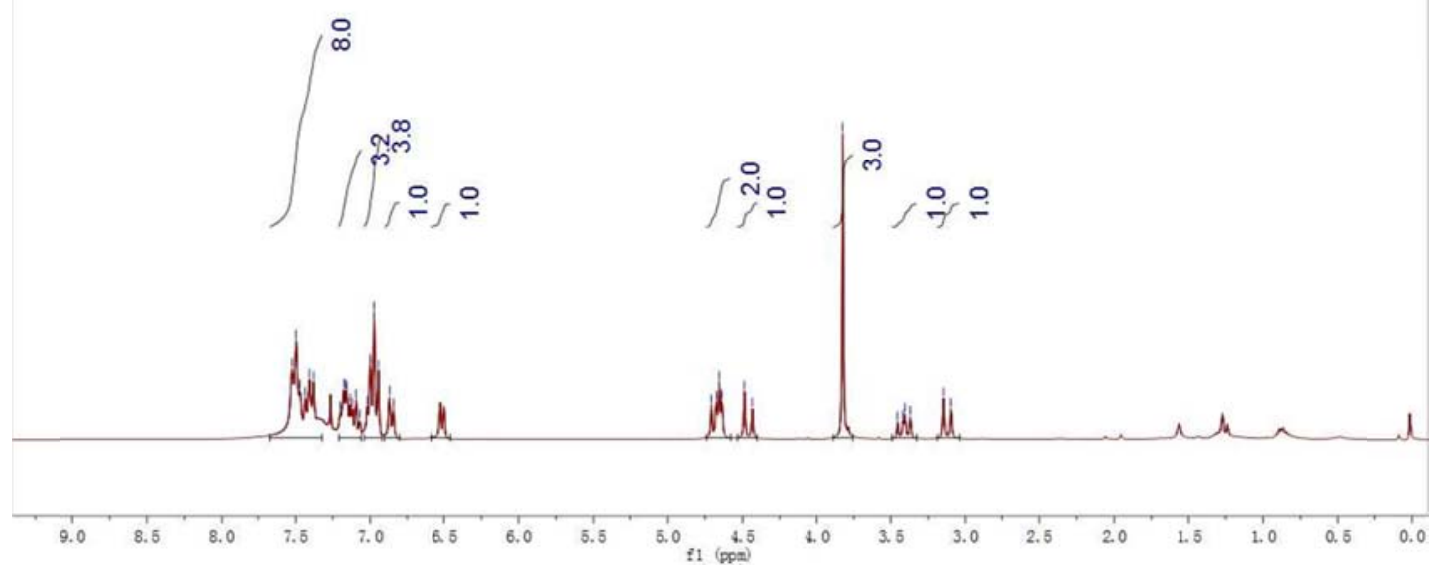

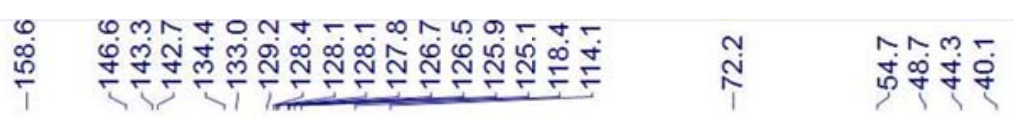<smiles>COc1ccc([C@H]2c3ccccc3CN(c3ccccc3)C23Oc2ccccc23)cc1</smiles>
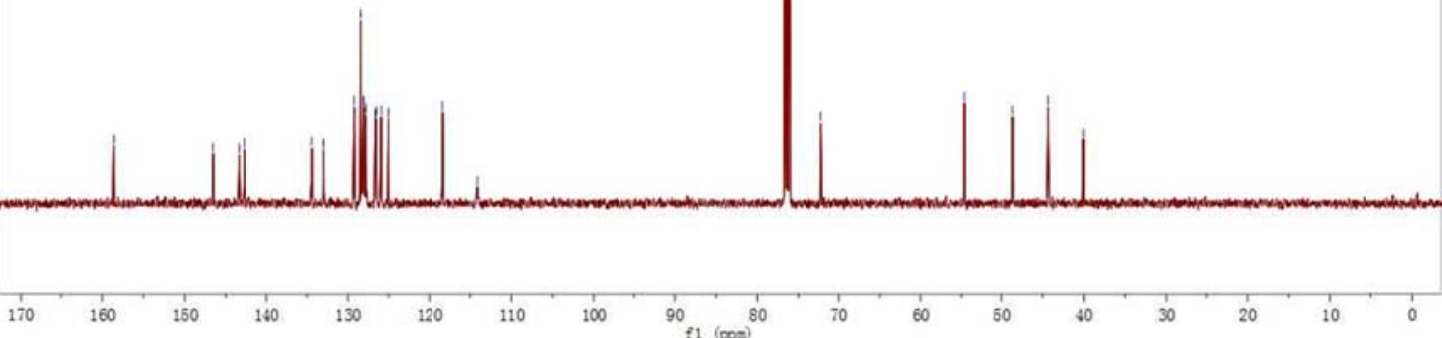


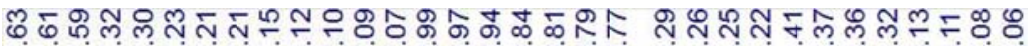

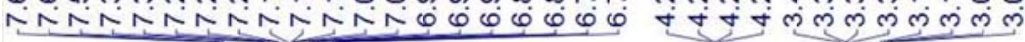<smiles>COc1ccc(C2CC(c3ccccc3)c3ccccc3OS2(=O)=O)cc1</smiles>

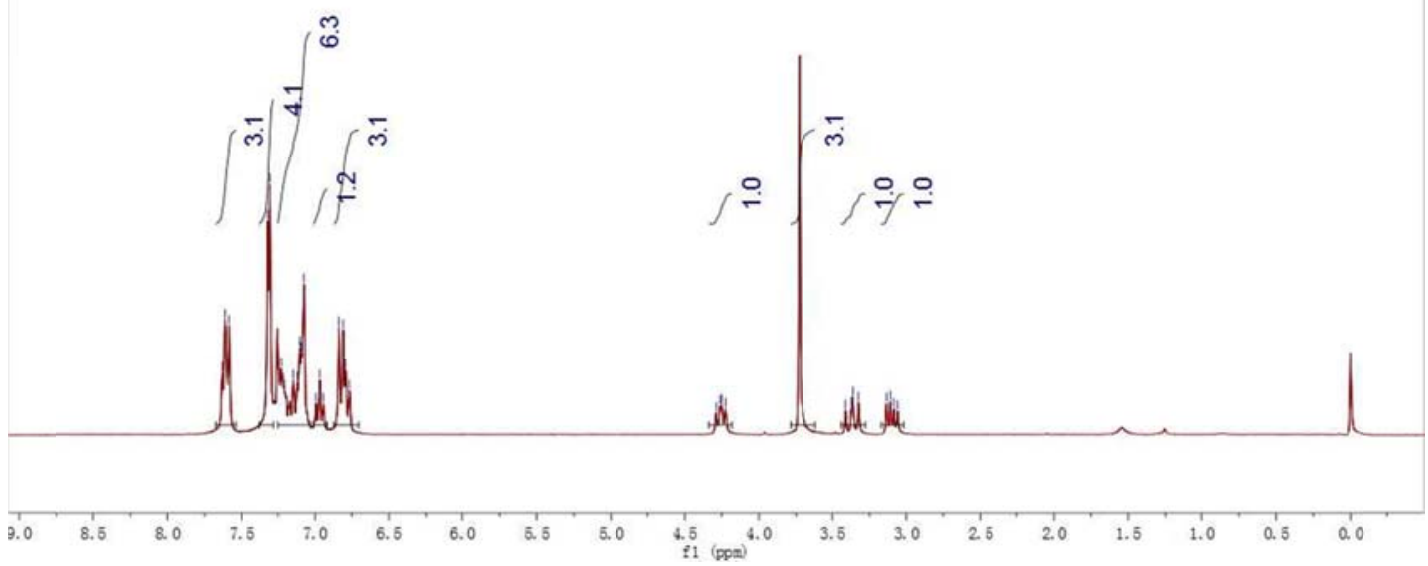

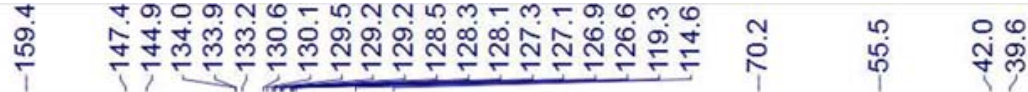
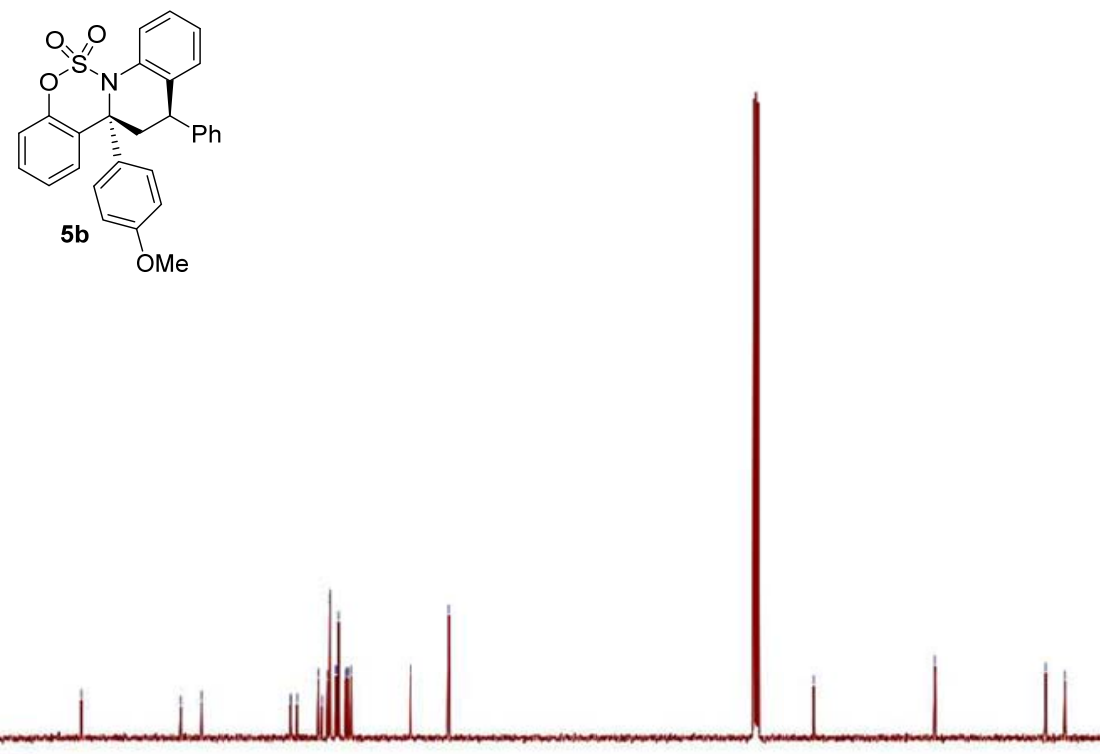

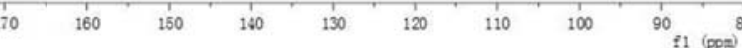



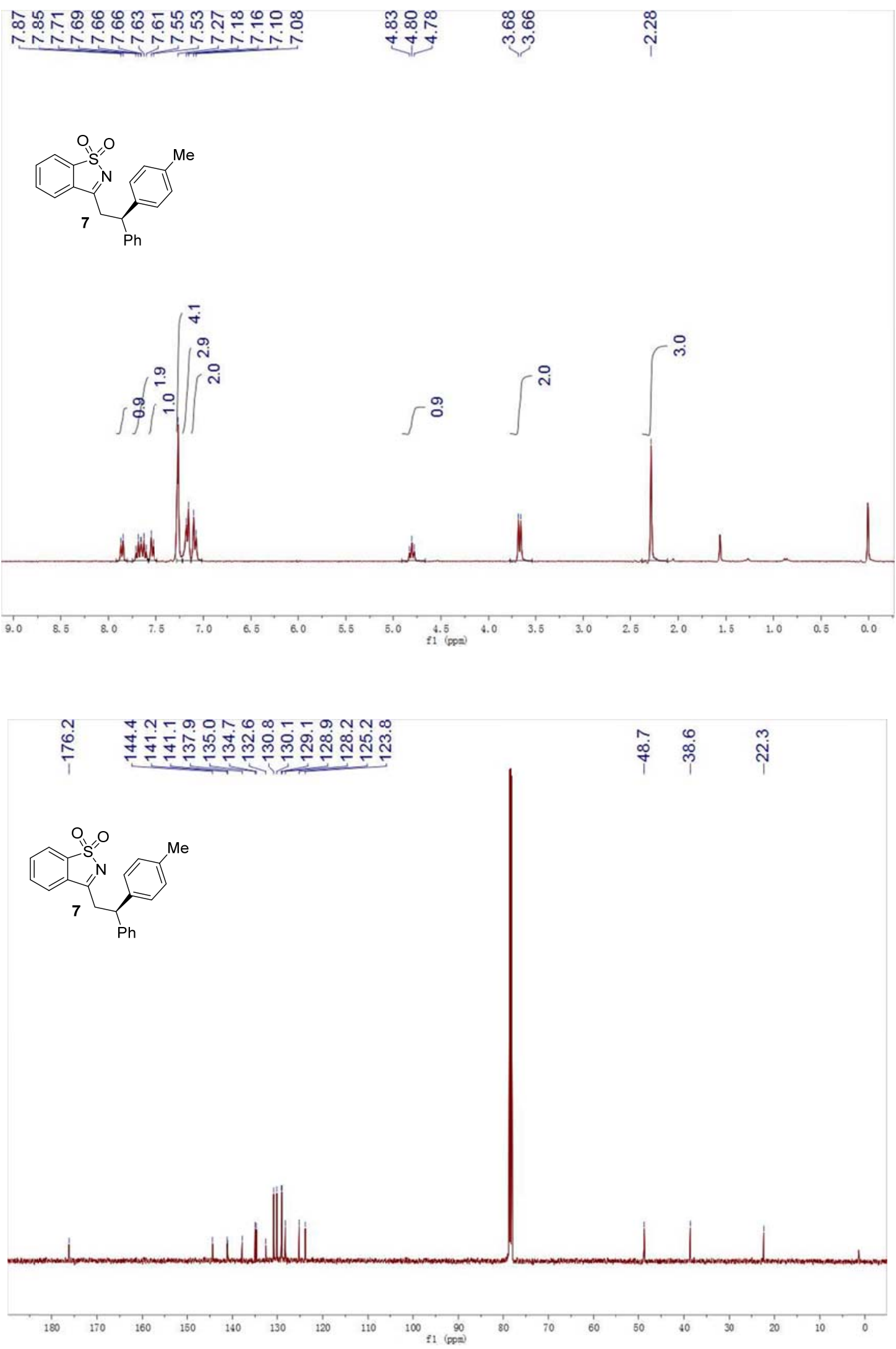


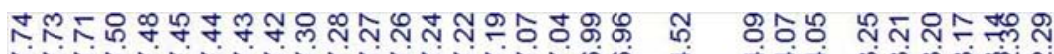

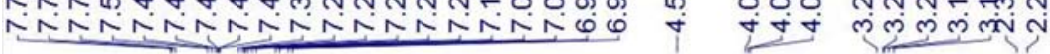<smiles></smiles>
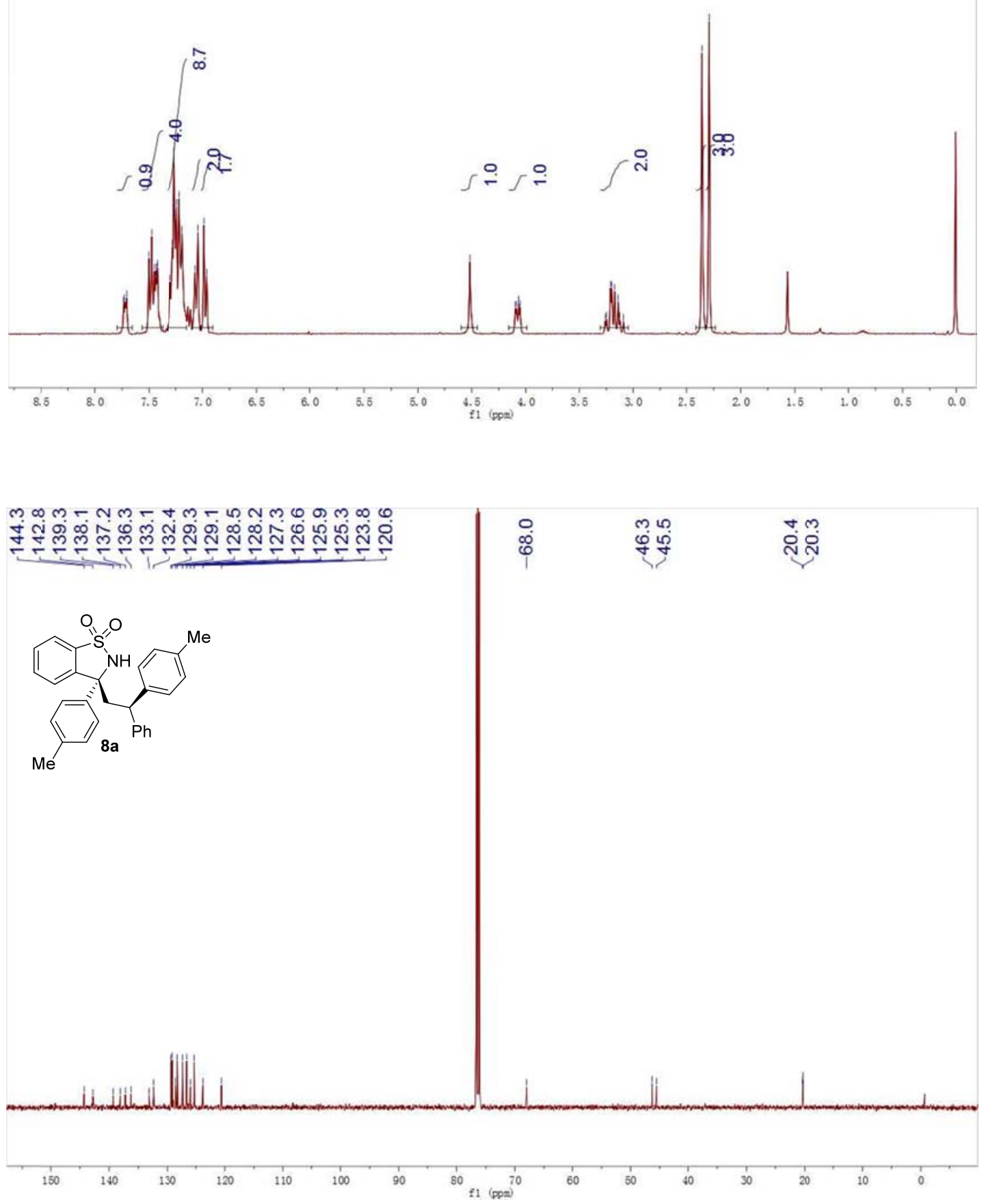